\title{
Unveiling the Molecular Structure of Antimalarial Drugs Chloroquine and Hydroxychloroquine in Solution Through Analysis of ${ }^{1} \mathrm{H}$ NMR Chemical Shifts
}

Isabel S. Hernandes ${ }^{\mathrm{a}}$, Haroldo C. Da Silva ${ }^{\mathrm{a}}$, Hélio F. Dos Santos ${ }^{\mathrm{b}}$, Wagner B. De Almeida ${ }^{\mathrm{a}, *}$

${ }^{a}$ Laboratório de Química Computacional e Modelagem Molecular (LQC-MM), Departamento de Química Inorgânica, Instituto de Química, Universidade Federal Fluminense (UFF), Outeiro de São João Batista s/n, Campus do Valonguinho, 24020-141, Centro, Niterói, RJ, Brazil.

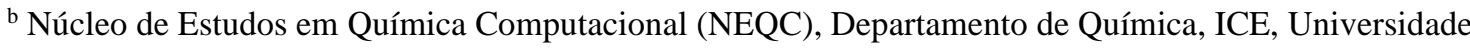
Federal de Juiz de Fora (UFJF), Campus Universitário, Martelos, Juiz de Fora, MG, 36036-330, Brazil.

\section{Supplementary Material}




\section{OPTIMIZED CARTESIAN COORDINATES}

\section{CHLOROQUINE}

\section{CQ-M1- Structure}

WB97x-D/6-31G(d,p) - Water fully optimized XYZ coordinates

\begin{tabular}{|c|c|c|c|}
\hline Atom & $\mathrm{X}$ & $\mathbf{Y}$ & $\mathbf{Z}$ \\
\hline C & 0.00000000 & 0.00000000 & 0.00000000 \\
\hline $\mathrm{C}$ & 0.00000000 & 0.00000000 & 1.36676119 \\
\hline $\mathrm{C}$ & 1.19762697 & 0.00000000 & 2.11005073 \\
\hline C & 2.39404767 & 0.04220731 & 1.44425159 \\
\hline C & 2.44763583 & 0.08850828 & 0.02885810 \\
\hline C & 1.23106560 & 0.02181129 & -0.70420932 \\
\hline $\mathrm{N}$ & 1.16798390 & -0.04237203 & -2.06463033 \\
\hline C & 2.31895272 & -0.06629468 & -2.70257649 \\
\hline C & 3.57827178 & 0.02289950 & -2.08821669 \\
\hline C & 3.67179883 & 0.14159766 & -0.71403215 \\
\hline $\mathrm{N}$ & 4.90382057 & 0.27481568 & -0.08488903 \\
\hline $\mathrm{C}$ & 5.19011912 & 1.46992639 & 0.74371403 \\
\hline $\mathrm{C}$ & 4.67883194 & 2.76621150 & 0.10134806 \\
\hline C & 6.68809207 & 1.49991565 & 1.01058064 \\
\hline $\mathrm{C}$ & 5.26846740 & 3.07405045 & -1.27280495 \\
\hline $\mathrm{C}$ & 4.47925302 & 4.16469392 & -1.98928455 \\
\hline $\mathrm{N}$ & 4.88593795 & 4.31876223 & -3.38340181 \\
\hline $\mathrm{C}$ & 4.24850048 & 3.29987271 & -4.22148304 \\
\hline $\mathrm{C}$ & 4.58672080 & 5.65772575 & -3.88960365 \\
\hline C & 5.03723599 & 3.00610134 & -5.48906767 \\
\hline C & 5.51921422 & 6.73010041 & -3.34433692 \\
\hline $\mathrm{H}$ & -0.91785716 & -0.02805074 & -0.57194611 \\
\hline $\mathrm{Cl}$ & -1.51798883 & -0.02266695 & 2.23687189 \\
\hline $\mathrm{H}$ & 1.16337535 & -0.04755685 & 3.19020394 \\
\hline $\mathrm{H}$ & 3.31369010 & -0.00422916 & 2.01188365 \\
\hline $\mathrm{H}$ & 2.27141243 & -0.13188083 & -3.78632885 \\
\hline $\mathrm{H}$ & 4.47191631 & 0.04559547 & -2.70226401 \\
\hline $\mathrm{H}$ & 4.68075984 & 1.35651952 & 1.70269468 \\
\hline $\mathrm{H}$ & 6.93288821 & 2.34450617 & 1.65670293 \\
\hline $\mathrm{H}$ & 7.01255312 & 0.57873325 & 1.49726110 \\
\hline $\mathrm{H}$ & 7.24868431 & 1.61702245 & 0.07846953 \\
\hline $\mathrm{H}$ & 4.87848453 & 3.59388076 & 0.78974390 \\
\hline $\mathrm{H}$ & 3.58740881 & 2.69904132 & 0.01383964 \\
\hline $\mathrm{H}$ & 6.31312617 & 3.38689513 & -1.19080742 \\
\hline $\mathrm{H}$ & 5.26629722 & 2.16911366 & -1.88888917 \\
\hline $\mathrm{H}$ & 4.63488563 & 5.11057742 & -1.46387916 \\
\hline $\mathrm{H}$ & 3.39748028 & 3.94591318 & -1.91813277 \\
\hline $\mathrm{H}$ & 3.21661068 & 3.60051303 & -4.47520294 \\
\hline $\mathrm{H}$ & 4.15807741 & 2.37654889 & -3.64143031 \\
\hline $\mathrm{H}$ & 3.53459509 & 5.92748877 & -3.68422789 \\
\hline $\mathrm{H}$ & 4.69113733 & 5.63275004 & -4.97724014 \\
\hline $\mathrm{H}$ & 4.53668003 & 2.23690823 & -6.08152280 \\
\hline $\mathrm{H}$ & 5.13860557 & 3.89337229 & -6.11861479 \\
\hline $\mathrm{H}$ & 6.04005509 & 2.65647199 & -5.23595137 \\
\hline $\mathrm{H}$ & 5.29554382 & 7.69224998 & -3.80999689 \\
\hline $\mathrm{H}$ & 5.41758808 & 6.85645080 & -2.26391571 \\
\hline $\mathrm{H}$ & 6.55787815 & 6.47289238 & -3.55955497 \\
\hline $\mathrm{H}$ & 5.65206711 & 0.14117209 & -0.75555267 \\
\hline
\end{tabular}




\section{CQ-M2 - Structure}

$\omega B 97 x-D / 6-31 G(d, p)-$ Water fully optimized XYZ coordinates

Atom

C

C

C

$\mathrm{C}$

C

C

$\mathrm{N}$

C

C

C

$\mathrm{N}$

C

C

C

C

C

$\mathrm{N}$

C

$\mathrm{C}$

C

C

$\mathrm{H}$

$\mathrm{Cl}$

$\mathrm{H}$

$\mathrm{H}$

$\mathrm{H}$

$\mathrm{H}$

$\mathrm{H}$

$\mathrm{H}$

$\mathrm{H}$

$\mathrm{H}$

$\mathrm{H}$

$\mathrm{H}$

$\mathrm{H}$

$\mathrm{H}$

$\mathrm{H}$

$\mathrm{H}$

$\mathrm{H}$

$\mathrm{H}$

$\mathrm{H}$

$\mathrm{H}$

$\mathrm{H}$

$\mathrm{H}$

$\mathrm{H}$

$\mathrm{H}$

$\mathrm{H}$

$\mathrm{H}$

$\mathrm{H}$

\section{$\mathrm{x}$}

$-5.08605800$

$-4.86844500$

$-3.57175500$

$-2.50008200$

$-2.67090300$

$-3.98877000$

$-4.27744300$

$-3.23972700$

$-1.89379200$

$-1.56881200$

$-0.29356000$

0.89445900

2.09604400

1.11341500

2.50683800

3.60700700

4.95995600

5.34930800

5.92999200

6.70653000

6.87655500

$-6.08422800$

$-6.22995400$

$-3.43224000$

$-1.51365500$

$-3.46804700$

$-1.12730400$

0.73547700

1.99190500

0.25046200

1.26952500

2.95156000

1.85756700

2.84188500

1.65225300

3.44535600

3.50481400

5.33597400

4.59167800

5.38362700

6.50528700

6.86699900

7.52348500

6.76194200

7.58820000

6.31025900

7.44002100

$-0.14708800$
Y

$-0.04323200$

$-1.39360700$

$-1.93765900$

$-1.08377800$

0.32139400

0.85094300

2.18414900

2.99269300

2.60272300

1.25041400

0.79309400

1.63995800

0.81264900

2.27905800

$-0.33945700$

$-1.20632400$

$-0.70716500$

$-1.00167000$

$-1.24976400$

$-0.44500700$

$-0.18465300$

0.37369300

$-2.49019300$

$-3.01048000$

$-1.52577600$

4.05585100

3.36364500

2. 43471700

2.92693500

2.88273100

1.51576200

1.48176500

0.40255900

0.05476700

$-0.98969900$

$-1.20535700$

$-2.25579000$

$-2.09523400$

$-0.57685500$

$-1.69565900$

$-2.06962200$

$-0.61316000$

$-0.93049400$

0.62892100

$-0.62407500$

0.58986100

0.29380900

$-0.15816700$
Z

0.01565200

0.03810700

$-0.02483000$

$-0.09552500$

$-0.10453800$

$-0.06385600$

$-0.10579400$

$-0.19099200$

$-0.22146200$

$-0.17036300$

$-0.21050700$

$-0.17671700$

$-0.64733500$

1.19752600

0.27669800

$-0.35139500$

$-0.10172100$

1.27761900

$-1.06481300$

1.68297200

$-1.60895400$

0.05668900

0.13260300

$-0.02643000$

$-0.15743400$

$-0.22494900$

$-0.27265500$

$-0.91431600$

1.16743800

1.49005400

1.96250900

$-0.77750400$

$-1.63371900$

1. 24127500

0.49871800

$-1.43343500$

$-0.01999000$

1.45409800

1.94079700

$-1.90055500$

$-0.60901800$

2.75062500

1.14432600

1. 48944100

$-2.31302000$

$-2.13196500$

$-0.80516400$

0.08932300 


\section{CQ-M3- Structure}

$\omega B 97 x-D / 6-31 G(d, p)$ - Water fully optimized XYZ coordinates

\section{Atom}

C

C

$\mathrm{C}$

$\mathrm{C}$

C

C

$\mathrm{N}$

C

C

C

$\mathrm{N}$

C

C

C

C

C

$\mathrm{N}$

C

C

C

C

$\mathrm{H}$

$\mathrm{Cl}$

$\mathrm{H}$

$\mathrm{H}$

$\mathrm{H}$

$\mathrm{H}$

$\mathrm{H}$

$\mathrm{H}$

$\mathrm{H}$

$\mathrm{H}$

$\mathrm{H}$

$\mathrm{H}$

$\mathrm{H}$

$\mathrm{H}$

$\mathrm{H}$

$\mathrm{H}$

$\mathrm{H}$

$\mathrm{H}$

$\mathrm{H}$

$\mathrm{H}$

$\mathrm{H}$

$\mathrm{H}$

$\mathrm{H}$

$\mathrm{H}$

$\mathrm{H}$

$\mathrm{H}$

$\mathrm{H}$

\section{$\mathrm{X}$}

4.52834100

5.00994500

4.22902000

2. 94724300

2.39687500

3.20448400

2.79003000

1.55268500

0.65150500

1.04820100

0.24705000

$-1.08238500$

$-2.19042300$

$-1.17761000$

$-2.18716700$

$-3.32511300$

$-4.64295100$

$-5.41920900$

$-5.38154900$

$-6.55939400$

$-4.68978800$

5.12761200

6.64025600

4.63932100

2.36839100

1.21546600

$-0.34643100$

$-1.18337100$

$-2.15717300$

$-0.40959600$

$-1.05031300$

$-2.10274300$

$-3.15899900$

$-1.22913600$

$-2.28874900$

$-3.23301900$

$-3.22182700$

$-5.81318500$

$-4.72980900$

$-5.54015400$

$-6.37157800$

$-7.04124100$

$-7.33020500$

$-6.17238900$

$-5.29896500$

$-3.71823900$

$-4.52534100$

0.59446300
$Y$

$-0.99598100$

0.11344700

1.27346800

1.28055200

0.15513800

$-1.00276700$

$-2.14194200$

$-2.12580600$

$-1.06100800$

0.12300900

1. 20659300

1.37892800

1.13370800

2.78305200

$-0.25619600$

$-0.41998000$

$-0.29914100$

0.81586300

$-1.55691300$

1. 24985800

$-2.63869100$

$-1.88805500$

0.10775600

2.13986900

2.18702500

$-3.03294800$

$-1.18627600$

0.65667300

2. 93711200

2. 94066600

3.53389600

1. 88864400

1.29974700

$-0.43596600$

$-1.01673200$

$-1.38436900$

0.34586400

0.59061500

1. 65789800

$-1.91891300$

$-1.37400300$

2. 14457200

0.48189600

1.47540100

$-3.54413500$

$-2.90442000$

$-2.28592600$

2.00335400
Z

$-0.03170600$

$-0.66901700$

$-0.82950300$

$-0.34081100$

0.31622500

0.47699200

1.10113300

1. 55724000

1.45238700

0.83495600

0.71913900

1.29461800

0.26758800

1.88176500

$-0.35718800$

$-1.36285000$

$-0.72725100$

$-1.26394000$

$-0.73363000$

$-0.35096200$

0.08934600

0.09419800

$-1.30750100$

$-1.33087100$

$-0.46738400$

2.05301200

1.84767700

2.10915900

2.33668500

2.64061900

1.09583500

$-0.52600100$

0.75277500

$-0.85837300$

0.42743700

$-1.87654000$

$-2.14133200$

$-2.27153200$

$-1.37469100$

$-1.76728800$

$-0.30855200$

$-0.75065600$

$-0.25084700$

0.64530800

0.12226000

$-0.33560400$

1. 11092400

0.21109100 


\section{CQ-M4- Structure}

$\omega B 97 x-D / 6-31 G(d, p)-$ Water fully optimized XYZ

coordinates

Atom

C

C

$\mathrm{C}$

C

C

C

$\mathrm{N}$

C

C

C

$\mathrm{N}$

C

C

C

C

C

$\mathrm{N}$

C

C

C

C

$\mathrm{H}$

CI

$\mathrm{H}$

$\mathrm{H}$

$\mathrm{H}$

$\mathrm{H}$

$\mathrm{H}$

$\mathrm{H}$

$\mathrm{H}$

$\mathrm{H}$

$\mathrm{H}$

$\mathrm{H}$

$\mathrm{H}$

$\mathrm{H}$

$\mathrm{H}$

$\mathrm{H}$

$\mathrm{H}$

$\mathrm{H}$

$\mathrm{H}$

$\mathrm{H}$

$\mathrm{H}$

$\mathrm{H}$

$\mathrm{H}$

$\mathrm{H}$

$\mathrm{H}$

$\mathrm{H}$

$\mathrm{H}$ $\mathrm{x}$

$-2.64546900$

$-3.09338300$

$-2.31548000$

$-1.05346200$

$-0.52493600$

$-1.35965000$

$-1.01833200$

0.15555400

1. 08518900

0.78918000

1. 72586400

2.07820800

2.01637700

3. 46367500

2.86586000

2.54415900

1.17408300

0.29245400

1.09534500

$-1.13599500$

1. 67199000

$-3.25203600$

$-4.67931000$

$-2.71658900$

$-0.46511400$

0.41511300

2. 05287300

1. 38524200

3. 76206400

3. 47040100

4. 20602500

2. 32105800

0.97843700

3. 93395600

2. 64452800

3. 25740100

2. 68224000

0.31724100

0.66048800

1. 59617900

0.03859900

$-1.80279200$

$-1.52966700$

$-1.16533500$

1.54603300

2.74083300

1.16667000

2.55539800
$\mathbf{Y}$

0.15990300

0.52086800

1. 30791400

1.67863600

1. 30058900

0.57635900

0.27831200

0.72120600

1. 38876800

1.65509800

2. 28817000

1. 94854100

0.44565800

2.53218100

$-0.43062300$

$-1.91443800$

$-2.21746800$

$-2.68925200$

$-3.04366200$

$-2.20104800$

$-2.35522700$

$-0.42574100$

0.00802600

1. 61263900

2. 29840100

0.50822200

1. 65546200

2.45648200

2.36073100

3. 60734300

2.06172900

0.30462300

0.10514400

$-0.25496300$

$-0.17100900$

$-2.52946500$

$-2.17568000$

$-3.78973300$

$-2.28773400$

$-4.01682600$

$-3.26201200$

$-2.61410100$

$-2.48423700$

$-1.11170500$

$-2.98070900$

$-2.15424700$

$-1.40224100$

2. 50126800
Z

0.92708900

$-0.31172900$

$-1.18363300$

$-0.79349300$

0.46615700

1. 36267300

2. 64776600

3. 05596400

2. 25192500

0.91987200

0.13471900

$-1.25880000$

$-1.56342900$

$-1.50857500$

$-0.64936000$

$-0.84421400$

$-0.43068400$

$-1.49111100$

0.76460500

$-1.28928600$

1.99088200

1.60507600

$-0.84325300$

$-2.13963000$

$-1.45647400$

4.08836000

2. 65791600

$-1.93695500$

$-2.54324400$

$-1.31875800$

$-0.85528200$

$-2.60513700$

$-1.49086500$

$-0.82016600$

0.39291600

$-0.28161900$

$-1.90049500$

$-1.58280100$

$-2.43732800$

0.60904200

0.94788400

$-2.04930200$

$-0.30872600$

$-1.35139600$

2.87778400

1.87163100

2.15643800

0.67060200 


\section{CQ-M5- Structure}

$\omega B 97 x-D / 6-31 G(d, p)-$ Water fully optimized XYZ coordinates

Atom

C

C

C

$\mathrm{C}$

$\mathrm{C}$

C

$\mathrm{N}$

C

C

C

$\mathrm{N}$

C

C

$\mathrm{C}$

C

C

$\mathrm{N}$

C

C

C

C

$\mathrm{H}$

$\mathrm{Cl}$

$\mathrm{H}$

$\mathrm{H}$

$\mathrm{H}$

$\mathrm{H}$

$\mathrm{H}$

$\mathrm{H}$

$\mathrm{H}$

$\mathrm{H}$

$\mathrm{H}$

$\mathrm{H}$

$\mathrm{H}$

$\mathrm{H}$

$\mathrm{H}$

$\mathrm{H}$

$\mathrm{H}$

$\mathrm{H}$

$\mathrm{H}$

$\mathrm{H}$

$\mathrm{H}$

$\mathrm{H}$

$\mathrm{H}$

$\mathrm{H}$

$\mathrm{H}$

$\mathrm{H}$

$\mathrm{H}$

\section{$\mathrm{x}$}

2.83940500

3.09846400

2.31462800

1.24353600

0.90467100

1.74035200

1.55766100

0.55238300

$-0.35527600$

$-0.23197200$

$-1.16615700$

$-1.86816200$

$-2.00360600$

$-3.22070800$

$-2.72497600$

$-2.04356400$

$-1.67084200$

$-2.82526700$

$-0.81317900$

$-2.48367200$

0.58961400

3.44989400

4.44938100

2.56678700

0.65580000

0.41323700

$-1.19206600$

$-1.30412400$

$-3.73311700$

$-3.09942500$

$-3.85366900$

$-2.52242200$

$-1.01472800$

$-3.77816600$

$-2.72650700$

$-2.68360600$

$-1.12219300$

$-3.22881000$

$-3.62115300$

$-1.28585300$

$-0.72875200$

$-3.35150000$

$-1.67813200$

$-2.15796800$

1.20894800

0.59536100

1.06063700

$-1.82860700$
$\mathbf{Y}$

$-0.06737500$

0.13850600

$-0.45481800$

$-1.22832100$

$-1.43788700$

$-0.87732900$

$-1.07107200$

$-1.85169100$

$-2.43204500$

$-2.20068400$

$-2.70837200$

$-1.86909300$

$-0.40833100$

$-2.51719200$

$-0.21543200$

0.84347400

2.04209200

2.76533400

2.92280200

3.48400900

2.37055400

0.37292000

1.14417500

$-0.30113200$

$-1.70107100$

$-2.02105200$

$-3.00504500$

$-1.89025800$

$-1.99295500$

$-3.56368700$

$-2.47271300$

0.14477500

0.05000100

0.02913000

$-1.14934400$

1.08289500

0.40262000

3.47414700

2.04703200

3.15888500

3.87130800

4.02015900

4.21038100

2.74931800

3.11946400

1.47289200

2.11205700

$-3.29711100$
Z

0.59575100

$-0.72899200$

$-1.73987300$

$-1.38057600$

$-0.02002500$

0.98289400

2.31869900

2.66207000

1.76646400

0.40589600

$-0.47917100$

$-1.48067400$

$-1.03796200$

$-1.74682500$

0.30519200

1. 17642800

0.43671800

$-0.09370000$

1.22237000

$-1.39190300$

1.44817900

1. 37257800

$-1.20425700$

$-2.78084200$

$-2.15508400$

3. 72691700

2.14788400

$-2.41794300$

$-2.55451400$

$-2.03366600$

$-0.85555600$

$-1.82869300$

$-0.97320600$

0.13874300

0.87856800

2.04498200

1.57251100

0.65018800

$-0.29729900$

2.19445600

0.68332900

$-1.78414100$

$-1.25181000$

$-2.13232100$

1. 94771800

2. 07201800

0.49564700

0.00989300 
Atom

C

C

C

$\mathrm{C}$

C

C

$\mathrm{N}$

C

$\mathrm{C}$

$\mathrm{C}$

$\mathrm{N}$

C

C

C

C

C

N

C

C

C

C

$\mathrm{H}$

$\mathrm{Cl}$

$\mathrm{H}$

H

$\mathrm{H}$

$\mathrm{H}$

$\mathrm{H}$

$\mathrm{H}$

$\mathrm{H}$

$\mathrm{H}$

$\mathrm{H}$

$\mathrm{H}$

$\mathrm{H}$

$\mathrm{H}$

$\mathrm{H}$

$\mathrm{H}$

$\mathrm{H}$

$\mathrm{H}$

$\mathrm{H}$

$\mathrm{H}$

$\mathrm{H}$

$\mathrm{H}$

$\mathrm{H}$

$\mathrm{H}$

$\mathrm{H}$

$\mathrm{H}$

$\mathrm{H}$ $\mathrm{x}$

$-2.94043900$

$-3.70934900$

$-3.47448300$

$-2.41773200$

$-1.55673900$

$-1.85789200$

$-1.17681200$

$-0.20557700$

0.21300700

$-0.42044400$

0.02627100

0.35064100

1.09610300

1.12535800

2.42656700

3.09799900

3.12421000

1.78747300

4.11902400

1.65546500

5.54565500

$-3.13983600$

$-5.04358800$

$-4.13182300$

$-2.26778500$

0.33321700

1.07285600

$-0.57891000$

1.32873600

0.55071900

2.08427400

1.26039700

0.44313900

3.10650400

2.28047400

4.12845900

2.59218600

1.47499000

1.08455000

4.02286300

3.91584000

0.59955600

2.16595100

2.06890700

6.24855800

5.83561800

5.65153800

0.78651300
$\mathbf{Y}$

$-1.35945500$

$-0.63371300$

0.73696000

1.34469700

0.62558200

$-0.73603500$

$-1.49020400$

$-0.88445200$

0.43480300

1.20696900

2.48603100

2.88717400

1.79212000

4.19676300

1.36759300

0.18695000

$-1.04312400$

$-1.63972800$

$-1.97453000$

$-2.80395300$

$-1.59330500$

$-2.40378700$

$-1.40283300$

1.29975000

2.40557200

$-1.48535800$

0.82330800

3.09046700

4.54547100

4.96119700

4.07014600

2.15791500

0.91749500

2.22222800

1.07811500

0.45060700

0.00062200

$-1.95575400$

$-0.86172700$

$-2.06476300$

$-2.96530100$

$-3.04034900$

$-3.70658400$

$-2.51816700$

$-2.33272300$

$-0.61947200$

$-1.55874500$

2.74243500
Z

0.29485200

$-0.57180500$

$-0.80252600$

$-0.17797400$

0.68855400

0.96882300

1.87659400

2.52902700

2. 30315700

1. 34402100

1.05533600

$-0.33168300$

$-1.10748400$

$-0.26990900$

$-0.48733200$

$-1.21142500$

$-0.41559300$

$-0.37895900$

$-0.94914400$

0.59662100

$-0.57190600$

0.49991100

$-1.40420300$

$-1.45155500$

$-0.32326100$

3. 25714400

2.83687800

$-0.86353300$

$-1.28264400$

0.25387200

0.24540800

$-2.12620900$

$-1.19890500$

$-0.47778400$

0.55873700

$-1.45556900$

$-2.17843100$

$-1.39291000$

$-0.06651700$

$-2.04659500$

$-0.53901700$

0.74272400

0.25414000

1.56862400

$-0.96359200$

$-0.97271400$

0.51483000

1. 67461400 


\section{CQ-M7- Structure}

$\omega B 97 x-D / 6-31 G(d, p)-$ Water fully optimized XYZ coordinates

Atom

C

$\mathrm{C}$

C

$\mathrm{C}$

C

C

$\mathrm{N}$

C

C

C

$\mathrm{N}$

C

C

$\mathrm{C}$

C

C

$\mathrm{N}$

C

C

C

C

$\mathrm{H}$

$\mathrm{Cl}$

$\mathrm{H}$

$\mathrm{H}$

$\mathrm{H}$

$\mathrm{H}$

$\mathrm{H}$

$\mathrm{H}$

$\mathrm{H}$

$\mathrm{H}$

$\mathrm{H}$

$\mathrm{H}$

$\mathrm{H}$

$\mathrm{H}$

$\mathrm{H}$

$\mathrm{H}$

$\mathrm{H}$

$\mathrm{H}$

$\mathrm{H}$

$\mathrm{H}$

$\mathrm{H}$

$\mathrm{H}$

$\mathrm{H}$

$\mathrm{H}$

$\mathrm{H}$

$\mathrm{H}$

$\mathrm{H}$

\section{$\mathrm{x}$}

$-3.73217000$

$-4.38948000$

$-3.86014200$

$-2.63663300$

$-1.89445800$

$-2.47145600$

$-1.89333500$

$-0.74591800$

$-0.07210800$

$-0.61226200$

0.04422400

0.44034400

1.16710300

1.26386000

2.43351200

3.09845100

4.17808200

3.74003800

5.13712200

2.76072700

5.87806200

$-4.15116900$

$-5.94875600$

$-4.42349000$

$-2.24721600$

$-0.28393800$

0.89304100

$-0.46294500$

1.57785800

0.67943700

2.16302500

1.40644000

0.46812300

3.14283500

2.19317800

3.46865500

2.33306200

4.64250000

3.28220600

5.86139200

4.67396400

2. 61644800

3.12548600

1.78235000

6.64973100

6.34988200

5.20432000

0.85332300
$\mathrm{Y}$

$-1.20404100$

$-0.07935200$

1.20935800

1.33427700

0.19848500

$-1.08933500$

$-2.24336100$

$-2.11754400$

$-0.90322000$

0.27823300

1.49105200

2. 30574300

1.48178900

3.48066600

0.77532800

0.00730000

$-0.91260200$

$-2.27443100$

$-0.38905200$

$-2.47467100$

0.82988100

$-2.19234400$

$-0.22546100$

2.08290700

2.32268400

$-3.03506100$

$-0.91044600$

2.71027000

4.10722500

4.08720000

3.13725200

2.14811800

0.73106200

1.50416900

0.08734400

0.74709000

$-0.57654900$

$-2.86780400$

$-2.67212400$

$-1.18384700$

$-0.13846600$

$-3.54005000$

$-2.00551200$

$-2.04608000$

1.14329500

0.59503300

1.67686900

1. 35848900
Z

$-0.15038000$

$-0.56410200$

$-0.35203200$

0.25139900

0.65915400

0.48901000

0.92888900

1.56008800

1. 76778300

1.29599100

1.47414100

0.30390600

$-0.76866600$

0.81418800

$-0.29372900$

$-1.44668300$

$-1.10272100$

$-0.82000800$

$-0.14119400$

0.34156400

$-0.67632100$

$-0.28329100$

$-1.34536200$

$-0.65190100$

0.45009300

1. 91480500

2. 26341400

$-0.15655700$

$-0.02206600$

1. 50707600

1.33318500

$-1.60500400$

$-1.15755000$

0.11402700

0.52066700

$-2.16899100$

$-1.97094000$

$-0.63942900$

$-1.73350500$

0.06077800

0.82862400

0.53721900

1.25992700

0.11100600

0.02915200

$-1.63347000$

$-0.82853100$

2.07045600 


\section{CQ-M8- Structure}

$\omega B 97 x-D / 6-31 G(d, p)-$ Water fully optimized XYZ coordinates

Atom

C

$\mathrm{C}$

C

C

C

C

$\mathrm{N}$

C

$\mathrm{C}$

C

$\mathrm{N}$

C

$\mathrm{C}$

C

C

C

$\mathrm{N}$

C

$\mathrm{C}$

C

C

$\mathrm{H}$

$\mathrm{Cl}$

$\mathrm{H}$

$\mathrm{H}$

$\mathrm{H}$

$\mathrm{H}$

$\mathrm{H}$

$\mathrm{H}$

$\mathrm{H}$

$\mathrm{H}$

$\mathrm{H}$

$\mathrm{H}$

$\mathrm{H}$

$\mathrm{H}$

$\mathrm{H}$

$\mathrm{H}$

$\mathrm{H}$

$\mathrm{H}$

$\mathrm{H}$

$\mathrm{H}$

$\mathrm{H}$

$\mathrm{H}$

$\mathrm{H}$

$\mathrm{H}$

$\mathrm{H}$

$\mathrm{H}$

$\mathrm{H}$

\section{$\mathrm{x}$}

$-4.92303000$

$-4.50410700$

$-3.13805700$

$-2.20568900$

$-2.58795000$

$-3.97050400$

$-4.45508000$

$-3.54989500$

$-2.16210000$

$-1.63843900$

$-0.30685400$

0.69802100

1.92297900

1.05589800

2.76497400

3.80037700

4.92252300

4.49172000

5.98044100

5.60937700

7.34784000

$-5.97265700$

$-5.68512700$

$-2.83806500$

$-1.15885600$

$-3.93114800$

$-1.51858500$

0.27455700

1.81056700

0.18140100

1.45568900

2.55245100

1.58616500

3.28907000

2.11592500

4.21312300

3.29165700

4.05890400

3.69405700

5.73391600

6.01764500

5.21549800

6.39961200

6.05749500

8.11980300

7.33468200

7.61890400

$-0.02989400$
$\mathrm{Y}$

$-0.40588900$

$-1.70642600$

$-2.04703900$

$-1.04234400$

0.31926500

0.64259300

1.91621700

2.87269200

2.68995900

1.40482200

1.15680900

2.19857100

1.59287900

2.89050100

0.66258000

$-0.08247600$

$-0.60375700$

$-1.58903500$

$-1.12383000$

$-2.02551000$

$-0.54071900$

$-0.14389700$

$-2.99453300$

$-3.08561100$

$-1.32072700$

3.88860600

3.55728800

2.94452100

3.65997900

3.36455300

2.16947400

2.41594200

1.05537100

1.23928900

$-0.04559100$

0.61746600

$-0.87930700$

$-2.47855900$

$-1.14271200$

$-0.88685600$

$-2.22106800$

$-2.69413800$

$-2.56032200$

$-1.15634600$

$-0.95757200$

0.54353800

$-0.75065400$

0.22593200
Z

$-0.01367000$

$-0.07174700$

$-0.06150100$

$-0.01409500$

0.02602300

0.04426800

0.11549600

0.16888300

0.13481100

0.04204100

$-0.03403700$

$-0.23391100$

$-0.92426000$

1.08036000

$-0.04978100$

$-0.88948000$

$-0.11627500$

0.86984400

$-0.99246800$

1.80660800

$-0.65886300$

$-0.01298400$

$-0.17387100$

$-0.09260800$

0.00952300

0.23597600

0.16379000

$-0.91827600$

0.90259300

1. 52916100

1.79603000

$-1.27437300$

$-1.81864200$

0.71879100

0.47797600

$-1.62449000$

$-1.46555600$

0.37458300

1. 46807300

$-2.03292400$

$-0.93624300$

2. 57511300

1.27417900

2. 29430800

$-1.31072400$

$-0.79019300$

0.37800500

$-0.29993700$ 


\section{CQ-M9- Structure}

$\omega B 97 x-D / 6-31 G(d, p)-$ Water fully optimized XYZ coordinates

\section{Atom}

C

C

$\mathrm{C}$

C

$\mathrm{C}$

C

$\mathrm{N}$

C

C

C

$\mathrm{N}$

C

C

C

C

C

$\mathrm{N}$

C

C

C

C

$\mathrm{H}$

$\mathrm{Cl}$

$\mathrm{H}$

$\mathrm{H}$

$\mathrm{H}$

$\mathrm{H}$

$\mathrm{H}$

$\mathrm{H}$

$\mathrm{H}$

$\mathrm{H}$

$\mathrm{H}$

$\mathrm{H}$

$\mathrm{H}$

$\mathrm{H}$

$\mathrm{H}$

$\mathrm{H}$

$\mathrm{H}$

$\mathrm{H}$

$\mathrm{H}$

$\mathrm{H}$

$\mathrm{H}$

$\mathrm{H}$

$\mathrm{H}$

$\mathrm{H}$

$\mathrm{H}$

$\mathrm{H}$

$\mathrm{H}$

\section{$\mathrm{x}$}

4. 91200000

4.82976400

3. 59239500

2. 44229300

2.47120300

3.72980000

3.87916900

2.77211500

1. 47786600

1. 28952400

0.03837800

$-1.07282400$

$-2.20349600$

$-1.55902600$

$-2.86242000$

$-3.99983200$

$-5.09492600$

$-5.89085100$

$-5.48538700$

$-5.58252000$

$-4.65867300$

5.86032000

6.28956800

3. 55903500

1. 50104200

2.89048600

0.64463900

$-0.73142500$

$-2.51202200$

$-0.86136900$

$-1.71153600$

$-2.95958500$

$-1.81538200$

$-3.26834500$

$-2.11449000$

$-3.60047100$

$-4.35225500$

$-6.95629000$

$-5.73875500$

$-5.43692000$

$-6.53804000$

$-6.27718800$

$-5.66691600$

$-4.56605100$

$-5.02755500$

$-3.60989000$

$-4.70077200$

0.09713700
$Y$

0.19482300

$-1.16807700$

$-1.83375200$

$-1.09163400$

0.32506400

0.97577000

2. 32853800

3.03814000

2.51398900

1.14392000

0.57642800

0.79253700

$-0.16601100$

2. 23852400

0.13565800

$-0.83185200$

$-0.83483400$

0.35370100

$-2.05225500$

1. 16048400

$-2.40038100$

0.70779500

$-2.12996500$

$-2.91475600$

$-1.62441900$

4.11771100

3.19305500

0.51192700

2. 27147200

2.87778000

2.65046600

$-0.13504700$

$-1.19412000$

1.15376100

0.11249600

$-1.85039100$

$-0.58160600$

0.08378700

0.99166000

$-2.89112900$

$-1.95404600$

1.99748800

0.52282500

1.56089100

$-3.31314200$

$-2.55396900$

$-1.58347900$

$-0.41041600$
Z

0.07150900

0.03331200

$-0.06912600$

$-0.14458900$

$-0.12523500$

$-0.00405000$

0.06387500

0.00319000

$-0.13156100$

$-0.19560800$

$-0.36791800$

0.58437900

0.19680000

0.64700800

$-1.14570500$

$-1.49522100$

$-0.56040500$

$-0.39805600$

0.10395800

0.86691200

1. 34236300

0.16144400

0.10587600

$-0.08200300$

$-0.20423800$

0.04872200

$-0.23057600$

1. 59022200

1.18114200

1.18684500

$-0.35335800$

0.98773500

0.19135700

$-1.13579900$

$-1.94604200$

$-1.54400400$

$-2.50933300$

$-0.39100300$

$-1.27695800$

$-0.60411600$

0.39161500

0.97815000

1. 75105400

0.83420000

1.81825500

1.07321200

2.06853400

$-0.57550600$ 


\section{CQ-M10- Structure}

$\omega B 97 x-D / 6-31 G(d, p)$ - Water fully optimized XYZ coordinates

\section{Atom}

C

C

$\mathrm{C}$

$\mathrm{C}$

$\mathrm{C}$

C

$\mathrm{N}$

C

C

C

$\mathrm{N}$

C

C

C

C

C

$\mathrm{N}$

C

C

C

C

$\mathrm{H}$

$\mathrm{Cl}$

$\mathrm{H}$

$\mathrm{H}$

$\mathrm{H}$

$\mathrm{H}$

$\mathrm{H}$

$\mathrm{H}$

$\mathrm{H}$

$\mathrm{H}$

$\mathrm{H}$

$\mathrm{H}$

$\mathrm{H}$

$\mathrm{H}$

$\mathrm{H}$

$\mathrm{H}$

$\mathrm{H}$

$\mathrm{H}$

$\mathrm{H}$

$\mathrm{H}$

$\mathrm{H}$

$\mathrm{H}$

$\mathrm{H}$

$\mathrm{H}$

$\mathrm{H}$

$\mathrm{H}$

$\mathrm{H}$

\section{$\mathrm{x}$}

2. 94751800

3. 41976100

2.80668800

1.68573600

1.13636700

1.80403600

1.42554100

0.39163900

$-0.36386000$

$-0.03741700$

$-0.81055000$

$-1.39570600$

$-1.83827200$

$-2.52514000$

$-2.84942000$

$-3.28513400$

$-2.26004600$

$-1.30413700$

$-1.56248800$

$-1.94620200$

$-2.46378400$

3.43334500

4.84349700

3.23085700

1.23614600

0.09159400

$-1.23977000$

$-0.64100200$

$-2.96484200$

$-2.14744500$

$-3.31794900$

$-2.24220700$

$-0.94168600$

$-3.75645300$

$-2.43370300$

$-3.96036000$

$-3.88687200$

$-0.66116600$

$-0.63312900$

$-0.74755200$

$-1.07227800$

$-1.17478600$

$-2.66155800$

$-2.46708000$

$-1.89292600$

$-2.86634800$

$-3.30241300$

$-1.52558500$

\section{Y}

$-0.42079800$

$-0.27962300$

0.58463300

1.27680100

1.14212500

0.31019800

0.18135600

0.90258900

1. 72888900

1.83854600

2. 61485300

2.08169000

0.61685500

3.01906100

0.36036700

$-1.10422500$

$-2.12383300$

$-1.83071200$

$-2.63029800$

$-1.54040700$

$-2.93256700$

$-1.06554200$

$-1.16543600$

0.70416500

1.96802700

0.80490200

2.23487900

2.12334600

2.68419900

4.03406900

3. 04258600

0.28737600

0.01863400

0.95017600

0.71450300

$-1.14602400$

$-1.40067200$

$-2.71143100$

$-0.99619900$

$-1.96237700$

$-3.56099500$

$-1.49495300$

$-2.32298200$

$-0.57929900$

$-3.46525800$

$-2.02786400$

$-3.56365200$

3.09742300
Z

$-0.77323900$

0.50093300

1. 43069600

1.05441000

$-0.24435100$

$-1.18567100$

$-2.48837700$

$-2.86849000$

$-2.02613300$

$-0.68455800$

0.16383900

1.41799300

1.28596500

1. 82710800

0.17084700

0.00839100

$-0.19135400$

$-1.25070100$

0.98843400

$-2.59955100$

2.17567500

$-1.49443600$

1.00179800

2.41837000

1.75356000

$-3.90843600$

$-2.41524800$

2.20684800

2.76775200

1.96067100

1.07278900

2.25037900

1. 10773000

0.34377900

$-0.77817500$

$-0.85321900$

0.86977200

$-1.34760500$

$-0.98068100$

1. 31610300

0.67880400

$-3.37123900$

$-2.86218800$

$-2.60483600$

2.93854000

2.63882400

1.87254700

$-0.36627000$ 


\section{CQ-M11- Structure}

$\omega B 97 x-D / 6-31 G(d, p)$ - Water fully optimized XYZ coordinates

\section{Atom}

C

C

$\mathrm{C}$

$\mathrm{C}$

$\mathrm{C}$

C

$\mathrm{N}$

C

C

C

$\mathrm{N}$

C

C

C

C

C

$\mathrm{N}$

C

C

C

C

$\mathrm{H}$

$\mathrm{Cl}$

$\mathrm{H}$

$\mathrm{H}$

$\mathrm{H}$

$\mathrm{H}$

$\mathrm{H}$

$\mathrm{H}$

$\mathrm{H}$

$\mathrm{H}$

$\mathrm{H}$

$\mathrm{H}$

$\mathrm{H}$

$\mathrm{H}$

$\mathrm{H}$

$\mathrm{H}$

$\mathrm{H}$

$\mathrm{H}$

$\mathrm{H}$

$\mathrm{H}$

$\mathrm{H}$

$\mathrm{H}$

$\mathrm{H}$

$\mathrm{H}$

$\mathrm{H}$

$\mathrm{H}$

$\mathrm{H}$

\section{$\mathrm{x}$}

$-4.16620300$

$-4.15422800$

$-3.02869000$

$-1.91476800$

$-1.87199200$

$-3.02336100$

$-3.11105200$

$-2.04470900$

$-0.84803600$

$-0.71836300$

0.42437300

1.59147000

2. 41088100

2.41738300

3.48698900

2. 95760300

3. 55815100

4.82963900

2. 67927500

5. 91191400

1.37630500

$-5.03449400$

$-5.57573400$

$-3.05180900$

$-1.06672400$

$-2.11581800$

$-0.03615700$

1. 21775300

3.30341000

1.83394400

2.75694600

2.89878500

1. 71626200

4.07708400

4.17534500

3.10069200

1.87959700

4.65652400

5.20071200

3.24219500

2. 45336800

6.84400600

5.65186200

6.08567800

0.88604100

1.56502200

0.67593700

0.42435900

\section{$Y$}

0.21752700

$-1.04200000$

$-1.54840100$

$-0.75644500$

0.54905500

1. 04895200

2. 29842300

3.06078000

2.68947300

1. 41161100

0.97664100

1.81691300

2.12105100

1.15592600

1.10298500

$-0.28647500$

$-1.39564600$

$-1.83090300$

$-2.56672800$

$-0.76576900$

$-2.35617900$

0.61216400

$-2.05484600$

$-2.54317600$

$-1.16291100$

4.06129700

3. 40228600

2.77467700

1. 75588500

1.05810000

0.16401500

3.08798500

2.25717800

1. 53565200

0.97320800

$-0.43858800$

$-0.30695900$

$-2.24622600$

$-2.65594700$

$-3.36436100$

$-2.93289700$

$-1.22703200$

0.01676800

$-0.29947700$

$-3.31739400$

$-1.89719300$

$-1.73153300$

0.07268700

\section{Z}

$-0.57109900$

$-0.04016100$

0.63545200

0.75297400

0.20981300

$-0.45495700$

$-0.99572700$

$-0.85556700$

$-0.23208100$

0.30391300

0.88294100

1.14057400

$-0.13281600$

2.23595400

$-0.51716400$

$-0.93257100$

$-0.18041100$

$-0.76572700$

$-0.13248200$

$-0.85735900$

0.62452900

$-1.08164900$

$-0.17326700$

1.06037300

1.29049600

$-1.27530400$

$-0.19125400$

1.52334600

2. 45101700

3.15324600

1. 91784900

0.02262600

$-0.96746600$

$-1.32898500$

0.32047400

$-2.01728900$

$-0.76328500$

$-1.77664500$

$-0.15019600$

0.36063100

$-1.14997700$

$-1.19002800$

$-1.57320900$

0.11478400

0.78939800

1.59989900

0.06543400

1. 32628200 


\section{CQ-M12- Structure}

$\omega B 97 x-D / 6-31 G(d, p)$ - Water fully optimized XYZ coordinates

\begin{tabular}{|c|c|c|c|}
\hline Atom & $\mathrm{x}$ & $\mathrm{Y}$ & z \\
\hline $\mathrm{C}$ & -4.62108500 & 0.21693100 & -0.65984800 \\
\hline C & -4.80530600 & -0.95588800 & 0.01955900 \\
\hline C & -3.77041800 & -1.55111100 & 0.76571900 \\
\hline C & -2.54873300 & -0.92924500 & 0.81367100 \\
\hline C & -2.31056300 & 0.28876200 & 0.13537600 \\
\hline $\mathrm{C}$ & -3.36171000 & 0.86770300 & -0.62406600 \\
\hline $\mathrm{N}$ & -3.23786300 & 2.02171900 & -1.34146000 \\
\hline C & -2.05104500 & 2.59462000 & -1.30797100 \\
\hline C & -0.93643900 & 2.13051800 & -0.59958400 \\
\hline C & -1.03703500 & 0.96639400 & 0.15554500 \\
\hline $\mathrm{N}$ & -0.00065700 & 0.45975100 & 0.86484200 \\
\hline $\mathrm{C}$ & 1.32103600 & 1.06608100 & 0.94607200 \\
\hline C & 2.31866400 & 0.01266400 & 1.43031200 \\
\hline C & 1.34679400 & 2.30010200 & 1.8464050 \\
\hline C & 2.56314200 & -1.12519500 & 0.4365140 \\
\hline $\mathrm{C}$ & 3.35180700 & -0.76042800 & -0.8341050 \\
\hline $\mathrm{N}$ & 4.80480500 & -0.62602300 & -0.7363820 \\
\hline C & 5.47780800 & -1.81630100 & -0.2302190 \\
\hline C & 5.30792300 & 0.55403600 & -0.0392350 \\
\hline C & 5.11713700 & -3.08954500 & -0.9824710 \\
\hline $\mathrm{C}$ & 4.72734600 & 1.87078700 & -0.5334250 \\
\hline $\mathrm{H}$ & -5.41564100 & 0.66825000 & -1.2397600 \\
\hline $\mathrm{Cl}$ & -6.36127700 & -1.75559300 & -0.0393110 \\
\hline $\mathrm{H}$ & -3.94023000 & -2.48702400 & 1.28134400 \\
\hline $\mathrm{H}$ & -1.75639800 & -1.41146900 & 1.37335600 \\
\hline $\mathrm{H}$ & -1.94764500 & 3.51411800 & -1.8786970 \\
\hline $\mathrm{H}$ & -0.01635400 & 2.69678100 & -0.63638900 \\
\hline $\mathrm{H}$ & 1.59021300 & 1.37006000 & -0.07291300 \\
\hline $\mathrm{H}$ & 2.32270100 & 2.78837900 & 1.77468600 \\
\hline $\mathrm{H}$ & 0.58069900 & 3.02037500 & 1.55443300 \\
\hline $\mathrm{H}$ & 1.17583200 & 2.01581300 & 2.88761600 \\
\hline $\mathrm{H}$ & 1.94092200 & -0.40047400 & 2.37408400 \\
\hline $\mathrm{H}$ & 3.25333000 & 0.51986800 & 1.6804200 \\
\hline $\mathrm{H}$ & 3.06969400 & -1.94738500 & 0.9523750 \\
\hline $\mathrm{H}$ & 1.59028300 & -1.52090600 & 0.1237120 \\
\hline $\mathrm{H}$ & 2.95837000 & 0.16212200 & -1.2706140 \\
\hline $\mathrm{H}$ & 3.15525800 & -1.53757400 & -1.5778870 \\
\hline $\mathrm{H}$ & 5.29274700 & -1.96298100 & 0.8503940 \\
\hline $\mathrm{H}$ & 6.55415000 & -1.63875700 & -0.3280280 \\
\hline $\mathrm{H}$ & 6.39160200 & 0.56993800 & -0.2019290 \\
\hline $\mathrm{H}$ & 5.17280100 & 0.47678100 & 1.0545050 \\
\hline $\mathrm{H}$ & 5.78289300 & -3.89999500 & -0.6802790 \\
\hline $\mathrm{H}$ & 4.09154900 & -3.40670700 & -0.7799120 \\
\hline $\mathrm{H}$ & 5.22241300 & -2.93851500 & -2.0594680 \\
\hline $\mathrm{H}$ & 5.30502500 & 2.70216400 & -0.1267200 \\
\hline $\mathrm{H}$ & 4.76707500 & 1.92331400 & -1.6238220 \\
\hline $\mathrm{H}$ & 3.68982600 & 2.01548800 & -0.2225060 \\
\hline $\mathrm{H}$ & -0.18933800 & -0.28003400 & 1.5217890 \\
\hline
\end{tabular}




\section{Atom}

C

C

C

$\mathrm{C}$

C

C

$\mathrm{N}$

C

C

C

$\mathrm{N}$

C

$\mathrm{C}$

C

C

C

$\mathrm{N}$

C

C

C

C

$\mathrm{H}$

$\mathrm{Cl}$

$\mathrm{H}$

$\mathrm{H}$

$\mathrm{H}$

$\mathrm{H}$

$\mathrm{H}$

$\mathrm{H}$

$\mathrm{H}$

$\mathrm{H}$

$\mathrm{H}$

$\mathrm{H}$

$\mathrm{H}$

$\mathrm{H}$

$\mathrm{H}$

$\mathrm{H}$

$\mathrm{H}$

$\mathrm{H}$

$\mathrm{H}$

$\mathrm{H}$

$\mathrm{H}$

$\mathrm{H}$

$\mathrm{H}$

$\mathrm{H}$

$\mathrm{H}$

$\mathrm{H}$

$\mathrm{H}$ $\mathrm{x}$

$-4.32702200$

$-4.35950700$

$-3.32382900$

$-2.25771300$

$-2.17240100$

$-3.22636600$

$-3.24777700$

$-2.19503800$

$-1.09072200$

$-1.05319400$

$-0.04438300$

1.33799100

1. 96271100

1. 50637800

2. 16092700

3. 34561300

4. 49607000

4.18937200

5.06978400

4.08324900

5.68950100

$-5.11889600$

$-5.71365500$

$-3.37036200$

$-1.47313300$

$-2.20348600$

$-0.29360300$

1.86665800

2.56640600

0.95397000

1. 18273000

1. 32389400

2. 92377400

2. 31210100

1. 23003000

3. 70458800

3. 00520700

3. 27084200

5.00218600

5.84874300

4. 32619500

3. 92926300

3. 24973200

5. 00482800

6.13174000

6.46720900

4. 94006600

$-0.11432400$
$Y$

0.07727300

1. 20734600

1. 51598400

0.65923300

$-0.52215200$

$-0.81190300$

$-1.89915500$

$-2.68812800$

$-2.50745200$

$-1.43303100$

$-1.22921200$

$-1.71459300$

$-1.26973600$

$-3.20790000$

0.24680600

0.75014000

1. 27941000

2. 54571400

0.30553700

3. 70813200

$-0.88870800$

$-0.15922300$

2. 30742000

2. 42213300

0.92688500

$-3.55195700$

$-3.23294500$

$-1.19507400$

$-3.41169600$

$-3.49906300$

$-3.83957700$

$-1.60533600$

$-1.78034700$

0.55531700

0.72824800

$-0.06299400$

1.53050600

2.48591900

2.75313900

0.81610300

$-0.05483400$

4. 64616400

3.59021000

3.79018000

$-1.57363200$

$-0.56682000$

$-1.44782200$

$-0.38229600$
Z

$-0.69629300$

0.07377800

0.97479700

1.08019400

0.30331800

$-0.60503500$

$-1.42700600$

$-1.36206100$

$-0.52131300$

0.36014200

1. 25215700

1.12356900

$-0.20699100$

1.39971000

$-0.26246600$

0.60168100

$-0.13735600$

$-0.80071800$

$-1.06569700$

0.18004800

$-0.35201500$

$-1.39395600$

$-0.06005600$

1. 56389300

1.77746800

$-2.02060200$

$-0.55397600$

1. 92814600

1.56669100

2.29468500

0.57102700

$-1.03128500$

$-0.33058000$

$-1.30046600$

0.05405700

1. 24749000

1. 28467400

$-1.40567800$

$-1.50295500$

$-1.63863200$

$-1.80023000$

$-0.35789400$

0.87815000

0.76357800

$-1.07824800$

0.34236100

0.20964200

1. 79114300 


\section{CQ-M14- Structure}

$\omega B 97 x-D / 6-31 G(d, p)$ - Water fully optimized XYZ coordinates

Atom

C

C

$\mathrm{C}$

$\mathrm{C}$

C

C

C

$\mathrm{N}$

C

C

$\mathrm{C}$

$\mathrm{N}$

$\mathrm{C}$

C

C

C

C

$\mathrm{N}$

C

$\mathrm{C}$

$\mathrm{C}$

C

$\mathrm{H}$

$\mathrm{Cl}$

$\mathrm{H}$

$\mathrm{H}$

$\mathrm{H}$

$\mathrm{H}$

$\mathrm{H}$

$\mathrm{H}$

$\mathrm{H}$

$\mathrm{H}$

$\mathrm{H}$

$\mathrm{H}$

$\mathrm{H}$

$\mathrm{H}$

$\mathrm{H}$

$\mathrm{H}$

$\mathrm{H}$

$\mathrm{H}$

$\mathrm{H}$

$\mathrm{H}$

$\mathrm{H}$

$\mathrm{H}$

$\mathrm{H}$

$\mathrm{H}$

$\mathrm{H}$

$\mathrm{H}$

$\mathrm{H}$

\section{$\mathrm{x}$}

2.61138900

$-4.60359300$

$-5.11593300$

$-4.42658600$

$-3.20133100$

$-2.62131000$

$-3.34355600$

$-2.89479300$

$-1.75168700$

$-0.95252700$

$-1.35900100$

$-0.62105900$

0.41935900

1. 72343900

$-0.06338500$

2.86939100

4. 22711400

5.17970600

4.87696200

6.56812900

5.78749000

6.99570400

$-5.13925300$

$-6.68234400$

$-4.87818500$

$-2.66541100$

$-1.40661200$

$-0.01634000$

0.62041900

0.69169700

$-0.99429700$

$-0.22087400$

2. 03149300

1.53675100

2.88316500

2.69928400

4.65942900

4.06875800

4. 90680400

3. 84479100

7.21339300

6.72074100

5.39616500

6.80293600

5.84636000

8.05475900

6.84961200

6.42856200

$-0.15335900$
$Y$

$-0.40628000$

0.95004600

$-0.31812400$

$-1.38466600$

$-1.14883300$

0.14323300

1. 21064000

2. 49815800

2.73490300

1.75098100

0.43663900

$-0.60913600$

$-1.27566700$

$-0.46892100$

$-1.56317200$

$-1.12986000$

$-0.51611200$

$-0.53243800$

0.55961700

$-0.53298700$

0.61254200

0.66654000

1. 76904400

$-0.63863500$

$-2.36761100$

$-1.94320600$

3. 76517500

2. 03292100

$-2.23376400$

$-2.14579500$

$-2.12947600$

$-0.63396300$

$-0.31807400$

0.52767400

$-2.20404600$

$-1.03169600$

$-1.07341800$

0.51504400

1. 53839000

0.42370900

$-0.60316800$

$-1.45548700$

1.33174900

0.92787700

$-0.36446200$

0.58699600

1. 61074300

0.71203900

$-0.23728400$
Z

0.85547000

$-0.58931000$

$-0.54913200$

0.06695200

0.63078500

0.60224600

0.00538900

$-0.00641800$

0.59611400

1. 21570400

1.20307800

1. 80863800

0.97832000

0.98236400

$-0.43611400$

0.21837100

0.58479800

$-0.52167900$

$-1.44604200$

$-0.05724200$

$-2.66377100$

0.79104200

$-1.05177500$

$-1.25961400$

0.10369600

1. 13568000

0.59026800

1. 68266900

1.46890700

$-0.96488000$

$-0.43967800$

$-0.99118400$

2. 02523900

0.56335600

0.42982000

$-0.85641200$

1. 42113600

0.95224500

$-0.93011900$

$-1.77970600$

$-0.93744700$

0.51375200

$-3.38690800$

$-2.40503600$

$-3.15015600$

1. 04489100

0.25738200

1. 72415400

2.62845900 


\section{CQ-M15- Structure}

$\omega B 97 x-D / 6-31 G(d, p)$ - Water fully optimized XYZ coordinates

Atom

C

C

$\mathrm{C}$

$\mathrm{C}$

C

C

$\mathrm{N}$

C

$\mathrm{C}$

$\mathrm{C}$

$\mathrm{N}$

C

$\mathrm{C}$

C

C

C

$\mathrm{N}$

C

C

$\mathrm{C}$

C

$\mathrm{H}$

$\mathrm{Cl}$

$\mathrm{H}$

$\mathrm{H}$

$\mathrm{H}$

$\mathrm{H}$

$\mathrm{H}$

$\mathrm{H}$

$\mathrm{H}$

$\mathrm{H}$

$\mathrm{H}$

$\mathrm{H}$

$\mathrm{H}$

$\mathrm{H}$

$\mathrm{H}$

$\mathrm{H}$

$\mathrm{H}$

$\mathrm{H}$

$\mathrm{H}$

$\mathrm{H}$

$\mathrm{H}$

$\mathrm{H}$

$\mathrm{H}$

$\mathrm{H}$

$\mathrm{H}$

$\mathrm{H}$

$\mathrm{H}$

\section{$\mathrm{x}$}

2.61138900

2.95581600

2.04502900

0.77349900

0.34639900

1.30424500

1.06530100

$-0.14094800$

$-1.17366800$

$-0.97841400$

$-2.02731800$

$-2.38385300$

$-2.14730300$

$-3.84454800$

$-2.88849800$

$-2.19836900$

$-0.84127300$

$-0.79587100$

0.05832600

$-1.09981400$

1.50629400

3.31695300

4.57035000

2.35615800

0.09560000

$-0.33412300$

$-2.14478100$

$-1.78937800$

$-4.14436900$

$-3.99622000$

$-4.49239500$

$-2.43254100$

$-1.08087400$

$-3.92859700$

$-2.92065400$

$-2.81400700$

$-2.12926100$

0.19023800

$-1.50405400$

$-0.24114100$

$-0.01746300$

$-1.07607000$

$-0.36168600$

$-2.09086000$

2.16979000

1.66776200

1.78301800

$-2.85224700$
$\mathrm{Y}$

$-0.40628000$

$-0.86230300$

$-1.57629000$

$-1.78584000$

$-1.29515200$

$-0.63462500$

$-0.21334800$

$-0.46347300$

$-1.06845300$

$-1.47031800$

$-2.05060500$

$-1.94732800$

$-0.55009200$

$-2.37051700$

0.57597300

1.91894800

1.93388200

2.16235200

2.78793400

3.58890100

2.65995300

0.11834600

$-0.58144000$

$-1.96417800$

$-2.36232900$

$-0.14758200$

$-1.19224900$

$-2.66696400$

$-2.36332300$

$-3.37559700$

$-1.67695100$

$-0.57514200$

$-0.31319300$

0.63821400

0.36992400

2.74196100

2.07685600

1.86240600

1.47051000

3.85059400

2.47489900

3.62930300

4.30511000

3.92189100

3.15304600

3.12183500

1.60462400

$-2.08735800$
Z

0.85547000

$-0.38441500$

$-1.18765800$

$-0.72264800$

0.53704400

1. 36034700

2. 63258900

3.10481000

2.38694100

1.06865600

0.40227700

$-1.02289900$

$-1.60469300$

$-1.14566700$

$-0.88488200$

$-1.13141400$

$-0.59887600$

0.84355200

$-1.36601600$

1. 31225000

$-0.91368800$

1.48611000

$-0.99996100$

$-2.14835500$

$-1.33554100$

4.12650300

2. 85232200

$-1.59474100$

$-2.19371300$

$-0.74602200$

$-0.60027200$

$-2.66236800$

$-1.56784200$

$-1.21946800$

0.19278100

$-0.73317400$

$-2.21305600$

1. 21145000

1.30912400

$-1.33870100$

$-2.41227500$

2.40405000

0.93289100

0.98385700

$-1.62680200$

0.06346900

$-0.83745000$

0.98408100 


\section{CQ-M16- Structure}

$\omega B 97 x-D / 6-31 G(d, p)$ - Water fully optimized XYZ coordinates

Atom

C

C

$\mathrm{C}$

C

C

C

$\mathrm{N}$

C

C

C

$\mathrm{N}$

C

C

C

C

C

$\mathrm{N}$

C

C

C

C

$\mathrm{H}$

$\mathrm{Cl}$

$\mathrm{H}$

$\mathrm{H}$

$\mathrm{H}$

$\mathrm{H}$

$\mathrm{H}$

$\mathrm{H}$

$\mathrm{H}$

$\mathrm{H}$

$\mathrm{H}$

$\mathrm{H}$

$\mathrm{H}$

$\mathrm{H}$

$\mathrm{H}$

$\mathrm{H}$

$\mathrm{H}$

$\mathrm{H}$

$\mathrm{H}$

$\mathrm{H}$

$\mathrm{H}$

$\mathrm{H}$

$\mathrm{H}$

$\mathrm{H}$

$\mathrm{H}$

$\mathrm{H}$

$\mathrm{H}$ $\mathrm{x}$

4.32915000

4.48306600

3.54484600

2. 45477100

2. 25520300

3.20815300

3. 12416100

2.07628400

1.06906700

1.13599400

0.21866600

$-0.91270800$

$-2.05536700$

$-1.36058400$

$-2.67077200$

$-3.72380000$

$-4.32241700$

$-3.43047700$

$-5.62716900$

$-3.71018700$

$-6.73175100$

5.04841400

5.87190300

3.68509300

1.73874500

2.00178800

0.26236800

$-0.55454100$

$-2.18952700$

$-0.54340400$

$-1.70256400$

$-2.82450600$

$-1.68043200$

$-3.14330500$

$-1.87361900$

$-4.51252900$

$-3.28505000$

$-3.48534000$

$-2.40414900$

$-5.55833900$

$-5.90056100$

$-3.00603500$

$-4.71874300$

$-3.60023700$

$-7.68801000$

$-6.55535600$

$-6.81763200$

0.33995400
$Y$

0.36540200

1.15823100

1.15852900

0.32980800

$-0.51835100$

$-0.49569200$

$-1.26090800$

$-2.05721800$

$-2.17772600$

$-1.42345200$

$-1.52967500$

$-2.44924700$

$-1.95425300$

$-2.69141800$

$-0.62653200$

$-0.15863100$

1.12724700

2. 22270600

1.29157700

3. 51758800

0.47009600

0.36758900

2. 21800800

1.80546100

0.35317600

$-2.66627200$

$-2.87509800$

$-3.40288100$

$-3.40054600$

$-3.10145500$

$-1.76480400$

$-2.73453600$

$-1.85238500$

$-0.71580300$

0.11957300

$-0.91516900$

$-0.12201100$

2. 39260300

1. 91532700

1.05767900

2. 34731100

4.29214800

3. 89263500

3.36313600

0.68240000

$-0.60471900$

0.71456100

$-0.97920000$
Z

$-0.85105500$

0.25189300

1.30118900

1.21143300

0.09681300

$-0.95685300$

$-2.08268300$

$-2.15700300$

$-1.19355300$

$-0.02517900$

0.96302600

0.95234800

0.05408000

2.38815800

0.48547300

$-0.51343800$

$-0.16203400$

$-0.54841300$

$-0.80380000$

0.20242000

$-0.15533000$

$-1.65979600$

0.36517900

2.15662700

2.02481700

$-3.05414400$

$-1.36912000$

0.54724700

2.40007700

2.98382700

2.85674400

0.03082400

$-0.97046200$

1. 46829800

0.58237200

$-0.54860500$

$-1.52860500$

$-1.63930300$

$-0.33763700$

$-1.88159100$

$-0.73760800$

$-0.10926000$

0.01417100

1. 27784100

$-0.63787200$

$-0.24133000$

0.90528800

1.79589000 


\section{CQ-M17- Structure}

$\omega B 97 x-D / 6-31 G(d, p)$ - Water fully optimized XYZ coordinates

Atom

C

$\mathrm{C}$

C

C

C

C

$\mathrm{N}$

C

C

$\mathrm{C}$

$\mathrm{N}$

C

C

C

C

C

$\mathrm{N}$

C

C

C

C

$\mathrm{H}$

$\mathrm{Cl}$

$\mathrm{H}$

$\mathrm{H}$

$\mathrm{H}$

$\mathrm{H}$

$\mathrm{H}$

$\mathrm{H}$

$\mathrm{H}$

$\mathrm{H}$

$\mathrm{H}$

$\mathrm{H}$

$\mathrm{H}$

$\mathrm{H}$

$\mathrm{H}$

$\mathrm{H}$

$\mathrm{H}$

$\mathrm{H}$

$\mathrm{H}$

$\mathrm{H}$

$\mathrm{H}$

$\mathrm{H}$

$\mathrm{H}$

$\mathrm{H}$

$\mathrm{H}$

$\mathrm{H}$

H $\mathrm{x}$

$-3.52105300$

$-3.48327400$

$-2.32278600$

$-1.19448700$

$-1.17000400$

$-2.36074100$

$-2.46994900$

$-1.37905200$

$-0.13519500$

0.01845400

1.19820200

2.43488200

3. 63869700

2. 53921900

3. 80332900

3.28870700

1.83071100

1. 39339500

1.14806100

2.02592200

1. 53408500

$-4.41855300$

$-4.91639100$

$-2.32900000$

$-0.30767300$

$-1.46713200$

0.69429300

2.45850000

3. 31136900

2. 81139500

1.59960100

4.52275800

3. 59567700

4.86784100

3.34001100

3. 71492500

3.68361400

1.60055000

0.30504100

0.07436500

1. 31263100

1.65560500

3.11390300

1.78340200

0.99004200

1.28144300

2.60179200

1. 32054600
$\mathbf{Y}$

0.57029100

$-0.62320700$

$-1.07031400$

$-0.29256000$

0.92607900

1.38302700

2.57846900

3. 31807100

2.96465600

1.73365900

1. 28203500

2.02659900

1. 10020400

3.20657300

0.00352600

$-1.38131000$

$-1.49489600$

$-2.60223200$

$-1.40778400$

$-3.97876000$

$-2.41864700$

0.92334000

$-1.62887200$

$-2.00507500$

$-0.62390500$

4.27863400

3.64722100

2.40989300

3. 89754500

2. 85212700

3.75643100

1.74280200

0.65172300

$-0.11672400$

0.32344000

$-2.12605800$

$-1.62130300$

$-2.29721500$

$-2.68165800$

$-1.47899800$

$-0.39614500$

$-4.67275700$

$-3.93300400$

$-4.39329200$

$-2.19065500$

$-3.44116300$

$-2.38098500$

0.26012100 z

0.33940200

$-0.32729700$

$-0.98831700$

$-0.93472900$

$-0.21942600$

0.39862900

1. 04638400

1.07177700

0.53293400

$-0.09915000$

$-0.60205400$

$-0.40594500$

$-0.57454100$

$-1.37440000$

0.49063400

0.09182700

0.01961000

$-0.84195200$

1. 31766900

$-0.61899100$

2.39976600

0.83039600

$-0.37705500$

$-1.53306100$

$-1.46124300$

1.57415200

0.64652400

0.62487100

$-1.03296500$

$-2.37063800$

$-1.44973400$

$-0.56676100$

$-1.57514900$

0.70801100

1. 42911600

0.78085200

$-0.90232000$

$-1.87421100$

$-0.74931500$

1.11193900

1.70890200

$-1.37780500$

$-0.71509100$

0.35909300

3. 31928400

2.11076600

2.63336500

$-0.61397100$ 


\section{CQ-M18- Structure}

$\omega B 97 x-D / 6-31 G(d, p)$ - Water fully optimized XYZ coordinates

Atom

C

C

C

$\mathrm{C}$

C

C

$\mathrm{N}$

C

C

C

$\mathrm{N}$

C

C

C

C

C

$\mathrm{N}$

C

C

C

C

$\mathrm{H}$

$\mathrm{Cl}$

$\mathrm{H}$

$\mathrm{H}$

$\mathrm{H}$

$\mathrm{H}$

$\mathrm{H}$

$\mathrm{H}$

$\mathrm{H}$

$\mathrm{H}$

$\mathrm{H}$

$\mathrm{H}$

$\mathrm{H}$

$\mathrm{H}$

$\mathrm{H}$

$\mathrm{H}$

$\mathrm{H}$

$\mathrm{H}$

$\mathrm{H}$

$\mathrm{H}$

$\mathrm{H}$

$\mathrm{H}$

$\mathrm{H}$

$\mathrm{H}$

$\mathrm{H}$

$\mathrm{H}$

$\mathrm{H}$ $\mathrm{x}$

$$
-4.82354700
$$

$-4.79045300$

$-3.60635900$

$-2.46210400$

$-2.44544700$

$-3.64399900$

$-3.74240000$

$-2.63371000$

$-1.39936300$

$-1.26628100$

$-0.09570000$

1. 11788600

2. 29070500

0.98954600

3. 65309400

4.78211900

4.81725700

5.15701400

5.68308200

3. 97306200

5.46451300

$-5.72931600$

$-6.24792000$

$-3.60799600$

$-1.56028400$

$-2.70784700$

$-0.56395100$

1. 27422500

1.83743400

0.96019300

0.07488000

2.15008000

2.29111100

3.73511700

3.76783300

5.75126400

4.62737900

5.44683600

6.02967200

5.46561200

6.74966600

4.23983400

3.13644000

3.62859600

6.02612400

4.40550900

5.80247000

$-0.10888600$
$Y$

0.05914900

$-1.21707100$

$-1.78377700$

$-1.02875600$

0.29936300

0.84607800

2.09674100

2.81122800

2.40571600

1.13373800

0.67103900

1.46572300

0.50526300

2. 47056000

1. 18886100

0.16262000

$-0.70016700$

$-2.07899000$

$-0.12450100$

$-2.80924800$

$-0.70879100$

0.49446700

$-2.18836800$

$-2.79970900$

$-1.48625400$

3.80552600

3.09008500

2. 00743100

3.15903900

1. 93800400

3.05793400

$-0.04811900$

$-0.23250900$

1. 84821500

1.82478800

0.66745900

$-0.45853800$

$-2.60143300$

$-2.12197900$

0.94631900

$-0.20915800$

$-3.83728000$

$-2.82442000$

$-2.31394100$

$-0.13210900$

$-0.68084000$

$-1.74477300$

$-0.20223600$ z

0.51658900

0.02413400

$-0.48211500$

$-0.49478000$

$-0.00716400$

0.52381700

1.05863300

1.05623300

0.53665300

$-0.01435400$

$-0.51308400$

$-0.66289400$

$-0.85209600$

$-1.80977200$

$-0.88091700$

$-0.94124900$

0.24056700

$-0.10136900$

1.26374000

$-0.72620300$

2. 65093800

0.91512300

0.02924200

$-0.85327200$

$-0.88302500$

1. 48974200

0.57407600

0.27786300

$-1.82755000$

$-2.76551100$

$-1.71496600$

$-1.79143400$

$-0.04416500$

$-1.75209500$

0.00720100

$-1.08243500$

$-1.82827300$

0.81391900

$-0.77661600$

1. 30064700

0.98033300

$-0.97960400$

$-0.02447900$

$-1.63955800$

3. 38778300

2.91093800

2.72647000

$-1.01618900$ 


\section{CQ-M19- Structure}

$\omega B 97 x-D / 6-31 G(d, p)$ - Water fully optimized XYZ coordinates

\section{Atom}

C

$\mathrm{C}$

C

$\mathrm{C}$

$\mathrm{C}$

C

$\mathrm{N}$

C

$\mathrm{C}$

C

$\mathrm{N}$

C

C

C

C

C

$\mathrm{N}$

C

C

C

C

$\mathrm{H}$

$\mathrm{Cl}$

$\mathrm{H}$

$\mathrm{H}$

$\mathrm{H}$

$\mathrm{H}$

$\mathrm{H}$

$\mathrm{H}$

$\mathrm{H}$

$\mathrm{H}$

$\mathrm{H}$

$\mathrm{H}$

$\mathrm{H}$

$\mathrm{H}$

$\mathrm{H}$

$\mathrm{H}$

$\mathrm{H}$

$\mathrm{H}$

$\mathrm{H}$

$\mathrm{H}$

$\mathrm{H}$

$\mathrm{H}$

$\mathrm{H}$

$\mathrm{H}$

$\mathrm{H}$

$\mathrm{H}$

$\mathrm{H}$ $\mathrm{x}$

2.80198800

3.01538100

2.38238900

1.50130200

1.23345200

1.90407900

1.71722500

0.88006100

0.13965100

0.25839200

$-0.60389100$

$-1.96680400$

$-2.60203200$

$-1.98156000$

$-2.10526500$

$-2.75380000$

$-2.19912100$

$-3.02574800$

$-0.76433500$

$-3.23705500$

$-0.19074900$

3.30017600

4.10350200

2.58425600

0.99138600

0.75454900

$-0.56257800$

$-2.60344500$

$-2.98357800$

$-1.30606500$

$-1.69535600$

$-2.55557300$

$-3.66650300$

$-2.31504800$

$-1.02152300$

$-2.68752700$

$-3.82258900$

$-4.00756400$

$-2.58862800$

$-0.52074300$

$-0.22870400$

$-3.94597500$

$-3.64508300$

$-2.30728900$

0.89291600

$-0.61535000$

$-0.37464100$

$-0.16461600$
$\mathrm{Y}$

$-0.15891000$

$-0.79547900$

$-0.38266100$

0.66502700

1.34867000

0.93575400

1.52511000

2.53916900

3.01095000

2.39041200

2.74911000

2.13674100

1.86828500

0.93238700

0.74089200

$-0.64062700$

$-1.48307400$

$-2.64844300$

$-1.73682000$

$-3.67184900$

$-2.16592300$

$-0.46827400$

$-2.16229800$

$-0.90132500$

0.95756400

3.02092000

3.82509300

2.90402000

0.49409400

0.14864800

1.23998300

2.81004800

1.69429800

1.07745800

0.62627500

$-1.12901500$

$-0.52089000$

$-2.27411800$

$-3.15765500$

$-2.49287400$

$-0.83106000$

$-4.43696100$

$-3.20259600$

$-4.17360600$

$-2.27985200$

$-3.11329300$

$-1.41051400$

2.60924400
Z

$-0.79884300$

0.39047200

1.58307200

1.53786100

0.32840500

$-0.85539200$

$-2.06946600$

$-2.11435500$

$-1.01735000$

0.20896100

1.25290200

1.30615700

$-0.06712100$

2.24194100

$-1.00232000$

$-0.86893400$

0.17751400

0.46408500

0.07591300

$-0.66090800$

$-1.28115100$

$-1.70653200$

0.44927100

2. 51533000

2. 44617200

$-3.07787200$

$-1.13958600$

1. 75620200

2.28367300

1. 90598800

3. 25165300

$-0.62407800$

0.13043100

$-2.02271100$

$-0.96585000$

$-1.85438500$

$-0.66183900$

0.78218100

1. 32894700

0.83055600

0.38029600

$-0.33802300$

$-1.55850000$

$-0.93462400$

$-1.18822800$

$-1.62844800$

$-2.05455600$

2.15659300 


\section{CQ-M20- Structure}

$\omega \mathrm{B} 97 \mathrm{x}-\mathrm{D} / 6-31 \mathrm{G}(\mathrm{d}, \mathrm{p})$ - Water fully optimized XYZ coordinates

Atom

$\mathrm{C}$

C

C

$\mathrm{C}$

C

C

$\mathrm{N}$

C

C

C

$\mathrm{N}$

C

C

C

C

C

$\mathrm{N}$

C

C

C

C

$\mathrm{H}$

$\mathrm{Cl}$

$\mathrm{H}$

$\mathrm{H}$

$\mathrm{H}$

$\mathrm{H}$

$\mathrm{H}$

$\mathrm{H}$

$\mathrm{H}$

$\mathrm{H}$

$\mathrm{H}$

$\mathrm{H}$

$\mathrm{H}$

$\mathrm{H}$

$\mathrm{H}$

$\mathrm{H}$

$\mathrm{H}$

$\mathrm{H}$

$\mathrm{H}$

$\mathrm{H}$

$\mathrm{H}$

$\mathrm{H}$

$\mathrm{H}$

$\mathrm{H}$

$\mathrm{H}$

$\mathrm{H}$

$\mathrm{H}$
3.69736600

4.58514800

4.25099800

2. 99751200

2.04281000

2.40045000

1. 57122200

0.38209200

$-0.08493200$

0.71936600

0.32133200

$-0.96930700$

$-1.96161000$

$-0.73126600$

$-2.45473200$

$-3.52266000$

$-4.04531900$

$-3.05106600$

$-4.73568000$

$-3.63549800$

$-6.11541400$

3. 95526200

6.18608700

4.97612900

2.76614300

$-0.29078000$

$-1.08646100$

$-1.36539500$

$-1.66189500$

0.00096500

$-0.35727100$

$-1.47346500$

$-2.82551300$

$-2.88791700$

$-1.61009700$

$-4.36572000$

$-3.13162200$

$-2.60846700$

$-2.22075700$

$-4.12536600$

$-4.85599500$

$-2.87200500$

$-4.47427700$

$-3.99298700$

$-6.61184500$

$-6.04899400$

$-6.73664800$

0.92938300
$-1.42841300$

$-0.60655700$

0.71376700

1.18247800

0.37290100

$-0.95616100$

$-1.82489800$

$-1.35100200$

$-0.06283700$

0.83508200

2.08545500

2. 70499300

2.52802000

4.18383000

1.09835900

0.67947400

$-0.67718100$

$-1.68923700$

$-0.92796900$

$-3.08731300$

$-1.54606900$

$-2.44317400$

$-1.19783500$

1.34299100

2. 20465100

$-2.03700900$

0.20077800

2.24089500

4. 67050400

4.30852000

4.68743400

2. 90992800

3.17766000

1.03097800

0.40279100

1. 37519900

0.80244600

$-1.40020900$

$-1.71860300$

$-1.56521100$

0.02183300

$-3.77496400$

$-3.08492400$

$-3.47420000$

$-1.69612000$

$-2.51066200$

$-0.88708700$

2.63505200
0.22151000

$-0.41565400$

$-0.76977600$

$-0.46786400$

0.19163400

0.54646300

1.19344900

1.50944700

1.23395200

0.53988500

0.21032600

0.49646000

$-0.66336200$

0.78476800

$-0.89695200$

0.12636100

0.01699900

0.35694200

$-1.25200400$

0.49533800

$-1.05504300$

0.49405500

$-0.80417000$

$-1.26819600$

$-0.74324100$

2.02073000

1.53993600

1.40330000

1.07971400

1.58388900

$-0.11219600$

$-1.56635800$

$-0.47705900$

$-1.89999900$

$-0.87485300$

0.04200000

1.14365000

1. 31645400

$-0.37236800$

$-1.90915200$

$-1.78139500$

0.86609000

1.19526600

$-0.46239100$

$-2.01777400$

$-0.54791400$

$-0.44430100$

$-0.37504500$ 


\section{CQ-M21- Structure}

$\omega B 97 x-D / 6-31 G(d, p)$ - Water fully optimized XYZ coordinates

\begin{tabular}{|c|c|c|c|}
\hline Atom & $\mathrm{x}$ & $\mathbf{Y}$ & $\mathbf{z}$ \\
\hline C & 3.09818100 & -1.46948000 & 0.54944100 \\
\hline C & 2.30865500 & -2.21873000 & -0.27790100 \\
\hline C & 1.33808500 & -1.63321800 & -1.12016800 \\
\hline $\mathrm{C}$ & 1.17772700 & -0.27468500 & -1.10367100 \\
\hline C & 1.95042400 & 0.54323500 & -0.24308900 \\
\hline C & 2.93914500 & -0.06045000 & 0.57838400 \\
\hline $\mathrm{N}$ & 3.77099900 & 0.65045600 & 1.39281200 \\
\hline C & 3.63913900 & 1.95684800 & 1.3820210 \\
\hline C & 2.68134400 & 2.65704600 & 0.6196860 \\
\hline $\mathrm{C}$ & 1.81329300 & 1.96196000 & -0.1893260 \\
\hline $\mathrm{N}$ & 0.83807500 & 2.61628700 & -0.9827320 \\
\hline C & -0.45479800 & 2.89310300 & -0.3108870 \\
\hline C & -1.20690100 & 1.59774300 & -0.0112260 \\
\hline $\mathrm{C}$ & -1.25754900 & 3.82145900 & -1.2135920 \\
\hline $\mathrm{C}$ & -2.61476300 & 1.82055000 & 0.5337200 \\
\hline $\mathrm{C}$ & -3.22313400 & 0.56077400 & 1.1580230 \\
\hline $\mathrm{N}$ & -2.94969400 & -0.66083600 & 0.40965000 \\
\hline C & -3.44147200 & -0.66647900 & -0.96370400 \\
\hline C & -3.31718100 & -1.85845600 & 1.16172700 \\
\hline C & -2.59570900 & -1.57303800 & -1.85344900 \\
\hline C & -4.79930100 & -1.99455900 & 1.53201600 \\
\hline $\mathrm{H}$ & 3.85489200 & -1.91674300 & 1.18052300 \\
\hline $\mathrm{Cl}$ & 2.51361900 & -3.95488600 & -0.33505100 \\
\hline $\mathrm{H}$ & 0.74108800 & -2.25624100 & -1.77447000 \\
\hline $\mathrm{H}$ & 0.46647200 & 0.20117500 & -1.76504800 \\
\hline $\mathrm{H}$ & 4.31195500 & 2.51779300 & 2.02517400 \\
\hline $\mathrm{H}$ & 2.62436500 & 3.73615700 & 0.68540200 \\
\hline $\mathrm{H}$ & -0.27005100 & 3.40720400 & 0.6460490 \\
\hline $\mathrm{H}$ & -2.16596500 & 4.15047500 & -0.7083960 \\
\hline $\mathrm{H}$ & -0.68251400 & 4.71155900 & -1.4784710 \\
\hline $\mathrm{H}$ & -1.54074200 & 3.30410200 & -2.1323420 \\
\hline $\mathrm{H}$ & -0.63053700 & 0.98703100 & 0.6921640 \\
\hline $\mathrm{H}$ & -1.27850500 & 1.01888200 & -0.9366820 \\
\hline $\mathrm{H}$ & -2.60365300 & 2.60911300 & 1.2952500 \\
\hline $\mathrm{H}$ & -3.25176000 & 2.18470300 & -0.2763040 \\
\hline $\mathrm{H}$ & -4.30126500 & 0.73579900 & 1.3190780 \\
\hline $\mathrm{H}$ & -2.77968400 & 0.41575100 & 2.1496490 \\
\hline $\mathrm{H}$ & -3.37758000 & 0.35261100 & -1.3531520 \\
\hline $\mathrm{H}$ & -4.50483200 & -0.95063700 & -1.03421500 \\
\hline $\mathrm{H}$ & -2.72249900 & -1.86904100 & 2.08072100 \\
\hline $\mathrm{H}$ & -3.00740800 & -2.73423000 & 0.58317500 \\
\hline $\mathrm{H}$ & -2.93736000 & -1.52366500 & -2.88911100 \\
\hline $\mathrm{H}$ & -1.54828100 & -1.25965800 & -1.80961600 \\
\hline $\mathrm{H}$ & -2.64789400 & -2.61690100 & -1.5340310 \\
\hline $\mathrm{H}$ & -4.97416600 & -2.94343300 & 2.04542800 \\
\hline $\mathrm{H}$ & -5.11926500 & -1.18689400 & 2.19648100 \\
\hline $\mathrm{H}$ & -5.43826100 & -1.97440900 & 0.6462300 \\
\hline $\mathrm{H}$ & 122515200 & 350948000 & $-1,2637560$ \\
\hline
\end{tabular}




\section{CQ-M22- Structure}

$\omega B 97 x-D / 6-31 G(d, p)$ - Water fully optimized XYZ coordinates

Atom

C

C

C

$\mathrm{C}$

$\mathrm{C}$

C

$\mathrm{N}$

C

C

C

$\mathrm{N}$

C

$\mathrm{C}$

$\mathrm{C}$

C

C

$\mathrm{N}$

C

C

C

C

$\mathrm{H}$

$\mathrm{Cl}$

$\mathrm{H}$

$\mathrm{H}$

$\mathrm{H}$

$\mathrm{H}$

$\mathrm{H}$

$\mathrm{H}$

$\mathrm{H}$

$\mathrm{H}$

$\mathrm{H}$

$\mathrm{H}$

$\mathrm{H}$

$\mathrm{H}$

$\mathrm{H}$

$\mathrm{H}$

$\mathrm{H}$

$\mathrm{H}$

$\mathrm{H}$

$\mathrm{H}$

$\mathrm{H}$

$\mathrm{H}$

$\mathrm{H}$

$\mathrm{H}$

$\mathrm{H}$

$\mathrm{H}$

$\mathrm{H}$

\section{$\mathrm{x}$}

3.50071000

3.49388700

2. 35823600

1.22020500

1.16526400

2. 33193800

2.40118700

1.29351200

0.06548000

$-0.04219700$

$-1.18184500$

$-2.47091800$

$-3.19704300$

$-3.31010900$

$-3.74419700$

$-2.74556700$

$-1.77776900$

$-2.38708600$

$-0.70956400$

$-3.20449300$

0.09115800

4.38163100

4.93936900

2.39542500

0.34812200

1.34972000

$-0.78909300$

$-2.28394800$

$-4.29649200$

$-2.81956100$

$-3.45330200$

$-4.03729900$

$-2.51529700$

$-4.27125800$

$-4.51105700$

$-2.17725600$

$-3.30261800$

$-1.56946800$

$-3.01771500$

$-0.03685100$

$-1.11154200$

$-3.55588100$

$-2.60828800$

$-4.08054600$

1.05336000

0.28239000

$-0.41670100$

$-1.27203800$
Y

0.57790800

$-0.64437300$

$-1.13234700$

$-0.36797900$

0.88352100

1.38092200

2.60300200

3. 31787500

2. 91743100

1.66925300

1.14117800

1.81719900

1.54635500

1.45682100

0.12940100

$-0.90839100$

$-1.41653600$

$-2.17833300$

$-2.18913600$

$-3.41072600$

$-1.38994500$

0.95812200

$-1.63424400$

$-2.08990100$

$-0.73638800$

4.29372500

3.57201100

2. 89316400

1.92264900

1.80314200

0.37670200

2.24645800

1.81279800

$-0.23952600$

0.20983700

$-0.43759500$

$-1.74152400$

$-2.47559300$

$-1.49886000$

$-2.52248800$

$-3.09398500$

$-3.91538300$

$-4.12396500$

$-3.14170800$

$-1.87514600$

$-0.38145900$

$-1.31371100$

0.13451900
Z

0.24935400

$-0.36496300$

$-1.03941700$

$-1.05547500$

$-0.39839600$

0.23499400

0.83754600

0.81820000

0.27153700

$-0.32984200$

$-0.86099700$

$-0.77621700$

0.55185700

$-1.99884700$

0.79284200

1. 31043300

0.33329000

$-0.76376900$

0.98052500

$-0.37511200$

2. 00183700

0.74928700

$-0.34262000$

$-1.54246400$

$-1.58287000$

1.29595800

0.37275000

$-0.82683100$

$-1.93151100$

$-2.90913400$

$-2.08629400$

0.60249200

1. 36872400

$-0.09334400$

1. 56853900

2.11573200

1.76651000

$-1.42861200$

$-1.34339200$

0.18403100

1. 46316400

$-1.27683000$

0.19843400

0.21988300

2.17297000

1.63009900

2.96542900

$-0.65430300$ 


\section{CQ-M23- Structure}

$\omega B 97 x-D / 6-31 G(d, p)$ - Water fully optimized XYZ coordinates

Atom

C

C

$\mathrm{C}$

$\mathrm{C}$

C

C

$\mathrm{N}$

C

C

C

$\mathrm{N}$

C

$\mathrm{C}$

C

C

C

$\mathrm{N}$

C

$\mathrm{C}$

C

C

$\mathrm{H}$

$\mathrm{Cl}$

$\mathrm{H}$

$\mathrm{H}$

$\mathrm{H}$

$\mathrm{H}$

$\mathrm{H}$

$\mathrm{H}$

$\mathrm{H}$

$\mathrm{H}$

$\mathrm{H}$

$\mathrm{H}$

$\mathrm{H}$

$\mathrm{H}$

$\mathrm{H}$

$\mathrm{H}$

$\mathrm{H}$

$\mathrm{H}$

$\mathrm{H}$

$\mathrm{H}$

$\mathrm{H}$

$\mathrm{H}$

$\mathrm{H}$

$\mathrm{H}$

$\mathrm{H}$

$\mathrm{H}$

$\mathrm{H}$

\section{$\mathrm{x}$}

4.77342400

5.14985700

4. 20863000

2.87879400

2. 43836400

3. 40290700

3. 09785300

1.81768400

0.76899600

1. 04934900

0.08718100

$-1.34062900$

$-1.90603200$

$-2.03387000$

$-3.01000200$

$-4.44168200$

$-4.82219200$

$-5.55792600$

$-5.52886400$

$-4.77071900$

$-4.62142400$

5.49609000

6.84356400

4.53623100

2.17049000

1.57366500

$-0.24772700$

$-1.46913300$

$-3.11005900$

$-1.65263700$

$-1.86038300$

$-1.07438500$

$-2.26415300$

$-2.91124500$

$-2.85296900$

$-4.53444900$

$-5.14766200$

$-6.51690200$

$-5.80481300$

$-5.92799900$

$-6.39745600$

$-5.30944900$

$-4.62513600$

$-3.78666300$

$-5.17172300$

$-3.77266500$

$-4.22483500$

0.34433800
$Y$

0.69396500

$-0.59445700$

$-1.59021900$

$-1.25593400$

0.06081700

1. 05518900

2.35963200

2.67293800

1.79039800

0.45103900

$-0.44999000$

$-0.15782500$

0.09776700

$-1.29884400$

1.16762700

0.63034300

$-0.29171800$

0.35143000

$-1.46912000$

1.44960400

$-2.41574300$

1.46133000

$-1.03438500$

$-2.59985800$

$-2.03624400$

3. 71283800

2.16075900

0.75924400

$-1.11877800$

$-1.39859800$

$-2.24160300$

0.41959200

$-0.85038300$

1.84133600

1.78850600

0.10812800

1. 47597100

0.76885600

$-0.42488600$

$-2.00067300$

$-1.18587300$

1.78219600

2.31907500

1.08198000

$-3.30900000$

$-2.71752200$

$-1.95155200$

$-1.41961600$
Z

0.22001900

$-0.04231700$

$-0.36408600$

$-0.40248300$

$-0.13148400$

0.17889500

0.43870400

0.38389000

0.09953900

$-0.16277100$

$-0.45901900$

$-0.48977400$

0.92784400

$-1.21986800$

0.99873300

1. 08889400

0.02062200

$-1.06080800$

0.51741200

$-1.76337300$

1.29328300

0.46303300

0.02201100

$-0.57324600$

$-0.65439200$

0.58790800

0.09906300

$-1.08088400$

$-1.22424600$

$-2.23781300$

$-0.68787900$

1.55922400

1. 34336400

0.14180900

1.88621600

2.04541900

1.14035600

$-0.70002500$

$-1.79136500$

$-0.35149900$

1.13849000

$-2.65268100$

$-1.11859800$

$-2.06894600$

1.59481700

0.67473800

2. 20048700

$-0.53895600$ 


\section{CQ-M24- Structure}

$\omega B 97 x-D / 6-31 G(d, p)$ - Water fully optimized XYZ coordinates

Atom

C

C

$\mathrm{C}$

$\mathrm{C}$

C

C

$\mathrm{N}$

C

C

C

$\mathrm{N}$

C

C

C

C

C

$\mathrm{N}$

C

C

C

$\mathrm{C}$

$\mathrm{H}$

$\mathrm{Cl}$

$\mathrm{H}$

$\mathrm{H}$

$\mathrm{H}$

$\mathrm{H}$

$\mathrm{H}$

$\mathrm{H}$

$\mathrm{H}$

$\mathrm{H}$

$\mathrm{H}$

$\mathrm{H}$

$\mathrm{H}$

$\mathrm{H}$

$\mathrm{H}$

$\mathrm{H}$

$\mathrm{H}$

$\mathrm{H}$

$\mathrm{H}$

$\mathrm{H}$

$\mathrm{H}$

$\mathrm{H}$

$\mathrm{H}$

$\mathrm{H}$

$\mathrm{H}$

$\mathrm{H}$

$\mathrm{H}$ $\mathrm{x}$

$-3.77281900$

$-3.57488400$

$-2.28564200$

$-1.20471800$

$-1.35706400$

$-2.66400000$

$-2.93134600$

$-1.87752500$

$-0.54322800$

$-0.23535500$

1. 02050500

2.19714900

3.36686500

2. 56842700

4.05696500

3.46045500

2.14720900

2.14478200

1. 62120100

3. 11716700

1. 12344500

$-4.76487700$

$-4.95659100$

$-2.15545200$

$-0.21434100$

$-2.08309200$

0.22931000

1. 95071000

1. 72373600

2.84697200

3. 40939100

4. 12030100

3. 03150100

4.16535900

5.07853900

3. 35229900

4.18356900

1. 11964900

2. 34067200

0.78873400

2.37692800

2. 97505500

2. 95345800

4.15655300

0.69324300

0.35122700

1. 92721300

1. 20348100
$Y$

0.45459000

$-0.89904200$

$-1.45990300$

$-0.61705300$

0.78728200

1.33596700

2. 67309400

3. 46543100

3. 05524100

1. 69388000

1. 22169700

2. 07776500

1. 38481100

2. 59208600

0.19433800

$-1.18478500$

$-1.44644600$

$-1.35933600$

$-2.73635000$

$-2.27750000$

$-2.68608800$

0.88445400

$-1.97433600$

$-2.53397200$

$-1.05010100$

4. 53353700

3.81081300

2. 94629500

3. 11507300

1.77262500

3. 28629400

2.16240400

1.07878100

0.36565000

0.14878000

$-1.29317700$

$-1.95176000$

$-1.56789100$

$-0.31988400$

$-3.01151600$

$-3.53011900$

$-2.17207000$

$-3.32591700$

$-2.02513900$

$-3.65004500$

$-1.92054200$

$-2.46799200$

0.21271200
Z

0.09212500

0.08969800

0.03151000

$-0.04057600$

$-0.05727000$

0.02275900

0.04055600

$-0.01522900$

$-0.10362500$

$-0.15211600$

$-0.29569400$

$-0.33137000$

$-1.04293900$

1.06125300

$-0.35756700$

$-0.63268800$

$-0.03537500$

1.43195100

$-0.50317600$

2.16777300

$-1.94158300$

0.14462300

0.15805600

0.04109400

$-0.08065100$

0.00847100

$-0.14081900$

$-0.95323700$

1.51379900

1.72781500

0.99871900

$-1.19579100$

$-2.03986800$

0.71855000

$-0.74333300$

$-1.71530700$

$-0.31349000$

1.75272300

1. 70940400

0.15325000

$-0.39722300$

3. 24512500

1. 90679800

1. 94210600

$-2.21818600$

$-2.05406700$

$-2.64731500$

$-0.33011600$ 


\section{CQ-M25- Structure}

$\omega B 97 x-D / 6-31 G(d, p)$ - Water fully optimized XYZ coordinates

Atom

$\mathrm{C}$

$\mathrm{C}$

C

$\mathrm{C}$

C

C

$\mathrm{N}$

C

C

C

$\mathrm{N}$

C

$\mathrm{C}$

C

C

C

$\mathrm{N}$

C

C

C

C

$\mathrm{H}$

$\mathrm{Cl}$

$\mathrm{H}$

$\mathrm{H}$

$\mathrm{H}$

$\mathrm{H}$

$\mathrm{H}$

$\mathrm{H}$

$\mathrm{H}$

$\mathrm{H}$

$\mathrm{H}$

$\mathrm{H}$

$\mathrm{H}$

$\mathrm{H}$

$\mathrm{H}$

$\mathrm{H}$

$\mathrm{H}$

$\mathrm{H}$

$\mathrm{H}$

$\mathrm{H}$

$\mathrm{H}$

$\mathrm{H}$

$\mathrm{H}$

$\mathrm{H}$

$\mathrm{H}$

$\mathrm{H}$

$\mathrm{H}$ $\mathrm{x}$

$-4.25467800$

$-4.47300200$

$-3.63040400$

$-2.54628600$

$-2.25963900$

$-3.15241300$

$-3.04359600$

$-2.06173200$

$-1.11360000$

$-1.16155000$

$-0.24513000$

0.56034300

1. 96349300

0.62563700

1. 99593000

3. 40658000

4. 34299500

5.40594300

4.82617400

5.59778600

5.65476700

$-4.91405000$

$-5.85425300$

$-3.85107100$

$-1.92283700$

$-1.98134200$

$-0.31365200$

0.06719200

1.22604200

$-0.37123300$

1. 08974200

2.44779400

2.56404700

1.33915500

1.61636600

3. 38170600

3. 75406700

6.35735700

5. 14491400

3. 94530300

5.41829800

6.39316400

5.85830500

4.67531100

5.88027600

5.11594300

6.60534300

0.31617400
$Y$

0.40177300

$-0.90616100$

$-1.59356700$

$-0.93984200$

0.40779100

1.09937900

2. 42490100

3.07872900

2. 50432300

1. 14810900

0.56250200

$-0.61790000$

$-0.19437000$

$-1.59247800$

0.68442600

1. 17897300

0.07690200

$-0.04853700$

0.10266900

$-1.47680300$

$-1.10948600$

0.94155900

$-1.75714100$

$-2.61799300$

$-1.46170600$

4.13896700

3.11216000

$-1.09788600$

$-2.46524500$

$-1.92678400$

$-1.11160800$

0.33495800

$-1.09194100$

1.55092100

0.11923900

1.85750000

1.78249400

0.34637700

0.57310700

0.15923200

1.01970000

$-1.51569400$

$-2.15673500$

$-1.83872300$

$-1.05833800$

$-2.04036100$

$-1.14361200$

1.24950500
Z

0.95670900

0.62432400

$-0.27238900$

$-0.80250600$

$-0.46971800$

0.39445000

0.69480300

0.10477100

$-0.75235700$

$-1.03052100$

$-1.87639400$

$-1.51140800$

$-1.07167700$

$-2.68141300$

0.17359800

0.47654000

0.72195300

$-0.27854700$

2.09901800

$-0.77407200$

2. 50150000

1. 62403600

1.29165200

$-0.54442900$

$-1.51769800$

0.33364900

$-1.15866800$

$-0.66075900$

$-2.41530200$

$-2.97572900$

$-3.54693200$

$-1.90585100$

$-0.89037700$

0.04478100

1. 03167900

1.33990200

$-0.37255200$

0.11093400

$-1.13877600$

2. 74319400

2.29022400

$-1.52440800$

0.03805300

$-1.23314800$

3. 57040400

2.30244600

1. 96549500

$-2.36858900$ 


\section{CQ-M26- Structure}

$\omega B 97 x-D / 6-31 G(d, p)$ - Water fully optimized XYZ coordinates

Atom

C

C

C

$\mathrm{C}$

C

C

$\mathrm{N}$

C

C

C

$\mathrm{N}$

C

$\mathrm{C}$

$\mathrm{C}$

C

C

$\mathrm{N}$

C

$\mathrm{C}$

C

C

$\mathrm{H}$

$\mathrm{Cl}$

$\mathrm{H}$

$\mathrm{H}$

$\mathrm{H}$

$\mathrm{H}$

$\mathrm{H}$

$\mathrm{H}$

$\mathrm{H}$

$\mathrm{H}$

$\mathrm{H}$

$\mathrm{H}$

$\mathrm{H}$

$\mathrm{H}$

$\mathrm{H}$

$\mathrm{H}$

$\mathrm{H}$

$\mathrm{H}$

$\mathrm{H}$

$\mathrm{H}$

$\mathrm{H}$

$\mathrm{H}$

$\mathrm{H}$

$\mathrm{H}$

$\mathrm{H}$

$\mathrm{H}$

$\mathrm{H}$ $\mathrm{x}$

$-3.53559000$

$-4.06290400$

$-3.39101600$

$-2.17375200$

$-1.58494600$

$-2.28026700$

$-1.82469200$

$-0.67247700$

0.10820100

$-0.31615700$

0.38771600

1. 73642600

2.77349200

2.05589700

2.93996600

3. 74454700

3. 06556300

3. 68841800

2.83491500

2.67346200

1.76103600

$-4.05478400$

$-5.61639400$

$-3.83614300$

$-1.67758300$

$-0.30423200$

1.03669600

1.72747700

3.06666000

1. 35181500

2. 00806100

3.73356100

2. 47319900

3. 42312200

1. 95482400

3. 88820600

4.75066300

4. 50509100

4. 14405900

3. 77147100

2.50918100

3.15399100

2.20521100

1.88298400

1. 62051700

2.02975100

0.81318600

0.10610400
$Y$

0.59976900

0.34395300

$-0.46252800$

$-0.99723900$

$-0.76160300$

0.04989800

0.33181200

$-0.21821500$

$-1.03844400$

$-1.32199800$

$-2.11356000$

$-2.60426300$

$-1.47408000$

$-3.69771400$

$-0.77582600$

0.51692500

1. 48710100

1.64254300

2.76891500

2.02371100

2. 67489900

1. 21096900

1.02015800

$-0.65182500$

$-1.62531500$

$-0.00406700$

$-1.43476200$

$-3.05425300$

$-4.07286000$

$-4.52767600$

$-3.30712000$

$-1.88586800$

$-0.72565800$

$-1.44345200$

$-0.51483400$

0.94456300

0.29744800

2. 38383400

0.68705300

3. 17057800

3. 48667500

2.10288400

2. 98788700

1. 26731200

3. 64383200

1. 94717200

2. 36033100

$-2.13521800$
Z

0.79501000

$-0.44122200$

$-1.38187000$

$-1.04429000$

0.22158100

1. 15869900

2.41408400

2.74006500

1.91614800

0.62199900

$-0.22363300$

0.04047500

0.03449000

$-0.96993400$

$-1.31024000$

$-1.18527300$

$-0.32245200$

0.99096300

$-0.98065200$

2.05926900

$-2.05747800$

1.52254200

$-0.87913700$

$-2.35098200$

$-1.77593300$

3. 74169400

2.30433600

1.03876900

$-0.80057900$

$-0.88307200$

$-1.99225800$

0.36470100

0.77336700

$-2.03245700$

$-1.71376800$

$-2.18310200$

$-0.80031000$

0.96155900

1. 26564100

$-1.41021400$

$-0.22251800$

3.03790200

1.84175100

2.11328800

$-2.54410300$

$-2.82797000$

$-1.61556900$

$-1.18981300$ 


\section{CQ-M27- Structure}

$\omega B 97 x-D / 6-31 G(d, p)$ - Water fully optimized XYZ coordinates

Atom

C

C

$\mathrm{C}$

$\mathrm{C}$

C

C

$\mathrm{N}$

C

$\mathrm{C}$

$\mathrm{C}$

$\mathrm{N}$

C

$\mathrm{C}$

C

C

C

$\mathrm{N}$

C

C

C

C

$\mathrm{H}$

$\mathrm{Cl}$

$\mathrm{H}$

$\mathrm{H}$

$\mathrm{H}$

$\mathrm{H}$

$\mathrm{H}$

$\mathrm{H}$

$\mathrm{H}$

$\mathrm{H}$

$\mathrm{H}$

$\mathrm{H}$

$\mathrm{H}$

$\mathrm{H}$

$\mathrm{H}$

$\mathrm{H}$

$\mathrm{H}$

$\mathrm{H}$

$\mathrm{H}$

$\mathrm{H}$

$\mathrm{H}$

$\mathrm{H}$

$\mathrm{H}$

$\mathrm{H}$

$\mathrm{H}$

$\mathrm{H}$

$\mathrm{H}$

\section{$\mathrm{x}$}

3.80545400

2.92927200

1.57744500

1.12034300

1.97499100

3.34782900

4.27420800

3.84061900

2.50183900

1.53819800

0.19794300

$-0.78340700$

$-2.18909400$

$-0.68024200$

$-2.45328200$

$-2.52162400$

$-3.79712600$

$-4.88706600$

$-4.23206400$

$-4.55116500$

$-3.20109400$

4.85082600

3.49221800

0.92331400

0.09711600

4.58378100

2.22916200

$-0.53800800$

$-1.34745300$

0.34093900

$-0.96867800$

$-2.33132500$

$-2.91617500$

$-3.38368700$

$-1.65760100$

$-1.76190300$

$-2.24623200$

$-5.22869600$

$-5.73747200$

$-5.12993200$

$-4.55047100$

$-5.45581700$

$-3.83471000$

$-4.12330700$

$-3.66053600$

$-2.81878300$

$-2.35178800$

0.10283800
$\mathrm{Y}$

$-1.00773000$

$-2.05483500$

$-1.87281800$

$-0.60478700$

0.51896000

0.30928600

1.30996900

2.51947900

2.84996300

1.86314800

2.13613400

1.96243100

1.87456200

3.09456500

0.59596900

$-0.70360200$

$-1.05364000$

$-1.23987700$

$-0.18991300$

$-2.20228000$

$-0.03197100$

$-1.15196500$

$-3.67850700$

$-2.72912900$

$-0.46086600$

3.31306400

3.88235200

1.02395300

2.90935100

3.18988300

4.04397700

2.74829400

1.97880100

0.70492800

0.49568800

$-0.69339400$

$-1.53719500$

$-0.28085400$

$-1.63410100$

$-0.65233100$

0.80559600

$-2.45325000$

$-1.77054100$

$-3.12472300$

0.44301300

$-1.00939000$

0.58794000

3.07497900
Z

0.21606300

0.14769200

$-0.21096200$

$-0.45606000$

$-0.34208300$

$-0.04611900$

$-0.01794000$

$-0.30200100$

$-0.57907500$

$-0.57743100$

$-0.84490000$

0.24768700

$-0.34847200$

1.26564100

$-1.14564400$

$-0.31819000$

0.30286800

$-0.64728600$

1.39506900

$-1.77757400$

2.50400000

0.45499500

0.47691600

$-0.30526800$

$-0.77615700$

$-0.29218200$

$-0.76336000$

0.75510400

2.11105500

1.64213200

0.80414800

$-0.99451400$

0.46091000

$-1.71242400$

$-1.89197700$

0.47027900

$-0.97019700$

$-1.07852100$

$-0.08094100$

1. 81924400

1.03636500

$-2.33407000$

$-2.48121300$

$-1.37716600$

3.37219000

2.80595300

2. 20577100

$-1.21944300$ 


\section{CQ-M28- Structure}

$\omega B 97 x-D / 6-31 G(d, p)$ - Water fully optimized XYZ coordinates

Atom

C

C

C

$\mathrm{C}$

C

C

N

C

$\mathrm{C}$

C

$\mathrm{N}$

C

$\mathrm{C}$

C

C

C

$\mathrm{N}$

C

C

C

C

$\mathrm{H}$

$\mathrm{Cl}$

$\mathrm{H}$

$\mathrm{H}$

$\mathrm{H}$

$\mathrm{H}$

$\mathrm{H}$

$\mathrm{H}$

$\mathrm{H}$

$\mathrm{H}$

$\mathrm{H}$

$\mathrm{H}$

$\mathrm{H}$

$\mathrm{H}$

$\mathrm{H}$

$\mathrm{H}$

$\mathrm{H}$

$\mathrm{H}$

$\mathrm{H}$

$\mathrm{H}$

$\mathrm{H}$

$\mathrm{H}$

$\mathrm{H}$

$\mathrm{H}$

$\mathrm{H}$

$\mathrm{H}$

$\mathrm{H}$ $\mathrm{x}$

4.45768900

4. 10581900

2.77146500

1.81184100

2. 11861700

3. 47396500

3. 91215200

2.99395600

1. 64484700

1. 16022000

$-0.18102300$

$-0.90317600$

$-2.36804500$

$-0.32100000$

$-3.08234700$

$-3.36281500$

$-4.51779300$

$-5.78466900$

$-4.40646300$

$-6.03642400$

$-3.14549000$

5.47830500

5.32439400

2. 51348100

0.79409000

3. 33586400

0.96933300

$-0.87528200$

$-0.84535500$

0.74193100

$-0.44516900$

$-2.39604000$

$-2.88899400$

$-4.02578100$

$-2.47566700$

$-2.47915100$

$-3.51282400$

$-5.86942700$

$-6.57714900$

$-5.27722100$

$-4.49217800$

$-7.06723900$

$-5.38080700$

$-5.87373800$

$-3.26816900$

$-2.95416800$

$-2.26298300$

$-0.64162500$
$Y$

$$
\begin{array}{r}
-0.02757100 \\
-1.34336000 \\
-1.73365400 \\
-0.76761400 \\
0.61724400 \\
0.98797200 \\
2.26699600 \\
3.20875300 \\
2.97958700 \\
1.68453300 \\
1.53345500 \\
0.47422800 \\
0.90642300 \\
0.17960800 \\
1.09454500
\end{array}
$$

$-0.18791400$

$-1.00297400$

$-0.27870400$

$-1.76788100$

0.50413900

$-2.61342600$

0.28044300

$-2.58432800$

$-2.78238400$

$-1.09274200$

4. 23286800

3. 82285600

$-0.43619500$

$-0.66842300$

$-0.06366900$

1. 04848100

1.84411500

0.16848100

1.62353500

1.75430400

$-0.83352000$

0.10175200

0.40219800

$-1.02513900$

$-2.43205600$

$-1.12484200$

0.86353500

1. 37398700

$-0.12770000$

$-3.36724900$

$-3.12200300$

$-2.01966300$

2. 42881400
Z

$-0.41014200$

$-0.33017700$

$-0.10106700$

0.04491400

$-0.01383600$

$-0.27166900$

$-0.41036600$

$-0.29979100$

$-0.03434600$

0.13449000

0.36098600

1. 06984100

1.18308800

2. 44930000

$-0.15805500$

$-0.96345600$

$-0.58815600$

$-0.59699400$

0.64929300

$-1.87673700$

0.74855000

$-0.59676000$

$-0.52458900$

$-0.05580300$

0.20230100

$-0.41906500$

0.05396600

0.46463700

2.89603600

2.39683900

3. 10016000

1.75053100

1.79699600

0.01086500

$-0.78840900$

$-0.96763900$

$-2.00672300$

0.26959000

$-0.47275700$

0.67887100

1. 54376600

$-1.89638100$

$-1.95930100$

$-2.75174400$

1.52912900

$-0.20072300$

1. 00543400

0.45771700 


\section{CQ-M29- Structure}

$\omega B 97 x-D / 6-31 G(d, p)$ - Water fully optimized XYZ coordinates

Atom

C

C

$\mathrm{C}$

$\mathrm{C}$

$\mathrm{C}$

C

$\mathrm{N}$

C

$\mathrm{C}$

C

$\mathrm{N}$

C

$\mathrm{C}$

$\mathrm{C}$

C

C

$\mathrm{N}$

C

C

C

C

$\mathrm{H}$

$\mathrm{Cl}$

$\mathrm{H}$

$\mathrm{H}$

$\mathrm{H}$

$\mathrm{H}$

$\mathrm{H}$

$\mathrm{H}$

$\mathrm{H}$

$\mathrm{H}$

$\mathrm{H}$

$\mathrm{H}$

$\mathrm{H}$

$\mathrm{H}$

$\mathrm{H}$

$\mathrm{H}$

$\mathrm{H}$

$\mathrm{H}$

$\mathrm{H}$

$\mathrm{H}$

$\mathrm{H}$

$\mathrm{H}$

$\mathrm{H}$

$\mathrm{H}$

$\mathrm{H}$

$\mathrm{H}$

$\mathrm{H}$

\section{$\mathrm{x}$}

3.86139000

4.66915900

4. 26784700

3. 02158300

2. 13288100

2.57419100

1.83587400

0.64912700

0.08819800

0.79903400

0.33343300

$-0.89285400$

$-2.07909000$

$-1.23094800$

$-2.54367300$

$-3.65920600$

$-4.35666900$

$-3.52357300$

$-5.25416700$

$-2.76424500$

$-6.22312000$

4. 17519800

6.25293300

4. 93852500

2. 74812900

0.05337800

$-0.91658400$

$-0.64040000$

$-1.89039500$

$-0.32562800$

$-1.74858100$

$-2.91230800$

$-1.79989600$

$-2.89672100$

$-1.69655300$

$-4.41472900$

$-3.23202900$

$-4.16177200$

$-2.81396500$

$-4.72058700$

$-5.82192300$

$-2.23339100$

$-3.45350200$

$-2.03310100$

$-6.97115800$

$-5.71019800$

$-6.73449700$

0.86916400
$Y$

1. 23205500

0.55202800

$-0.66618400$

$-1.15932200$

$-0.47592700$

0.72934300

1. 44956400

0.95379500

$-0.20739500$

$-0.95097700$

$-2.05798400$

$-2.83735900$

$-2.31139800$

$-3.08528100$

$-0.89787100$

$-0.44295400$

0.79072300

1. 98958500

0.67393000

2. 32755800

1. 84575300

2.15712400

1. 18408100

$-1.20379900$

$-2.11217100$

1.51864500

$-0.47190100$

$-3.81521500$

$-3.95156700$

$-3.29634000$

$-2.24890700$

$-3.01219900$

$-2.35029100$

$-0.83407900$

$-0.21279000$

$-1.23634500$

$-0.34056800$

2.84564400

1.88451100

0.56828200

$-0.25180300$

3.27370800

2.44969300

1.56424300

1.65367100

2.77580700

1. 99376400

$-2.36888000$
Z

0.46494400

$-0.40352300$

$-0.98255000$

$-0.69040700$

0.17358400

0.78235500

1. 67434400

1. 96085300

1. 41709400

0.47893500

$-0.15053400$

0.05710700

$-0.76687300$

1.52681600

$-0.43745700$

$-1.38653900$

$-1.06334100$

$-1.06316500$

0.07556000

0.22679500

0.17160200

0.93020400

$-0.79855100$

$-1.63905100$

$-1.12716700$

2.67383000

1.70535000

$-0.36289600$

1. 59079800

2.09837800

1.99598100

$-0.63845700$

$-1.82599700$

0.59903500

$-0.52855300$

$-1.44547600$

$-2.39213300$

$-1.31076900$

$-1.88870900$

1.03638100

$-0.06299800$

0.09733900

1. 06654900

0.50051000

0.94309200

0.43077500

$-0.78240100$

$-0.94232100$ 


\section{CQ-M30- Structure}

$\omega B 97 x-D / 6-31 G(d, p)$ - Water fully optimized XYZ coordinates

Atom

C

$\mathrm{C}$

C

C

C

C

$\mathrm{N}$

C

C

C

$\mathrm{N}$

C

$\mathrm{C}$

C

C

C

$\mathrm{N}$

C

C

C

C

$\mathrm{H}$

$\mathrm{Cl}$

$\mathrm{H}$

$\mathrm{H}$

$\mathrm{H}$

$\mathrm{H}$

$\mathrm{H}$

$\mathrm{H}$

$\mathrm{H}$

$\mathrm{H}$

$\mathrm{H}$

$\mathrm{H}$

$\mathrm{H}$

$\mathrm{H}$

$\mathrm{H}$

$\mathrm{H}$

$\mathrm{H}$

$\mathrm{H}$

$\mathrm{H}$

$\mathrm{H}$

$\mathrm{H}$

$\mathrm{H}$

$\mathrm{H}$

$\mathrm{H}$

$\mathrm{H}$

$\mathrm{H}$

$\mathrm{H}$

\section{$\mathrm{x}$}

3.51165800

3.04018200

1.81506200

1.10119100

1.56503700

2.77027600

3.25672700

2.51846000

1.32270900

0.83754200

$-0.26355700$

$-1.52249300$

$-1.98672100$

$-1.50047900$

$-1.99551300$

$-2.79953000$

$-2.90465100$

$-3.96037400$

$-3.12940600$

$-3.80050600$

$-1.87672200$

4.43957900

3.96635500

1.43723500

0.14331800

2.89547800

0.82534200

$-2.24547600$

$-2.52301600$

$-1.06403900$

$-0.93572700$

$-1.34129300$

$-2.99230500$

$-0.97417600$

$-2.40368700$

$-2.30576100$

$-3.79811200$

$-3.92609100$

$-4.95586400$

$-3.46047700$

$-3.94834500$

$-4.59157100$

$-2.83603400$

$-3.85564400$

$-2.04878700$

$-1.04249900$

$-1.58327100$

$-0.41868700$
$\mathrm{Y}$

$-0.54783800$

$-1.66655100$

$-1.66478700$

$-0.49766800$

0.69719200

0.66009000

1.72238300

2.81374200

2.97993700

1.93889800

2.04933000

2.70904800

2.29832500

4.22626900

0.78995700

0.00285800

$-1.41282200$

$-1.62304300$

$-2.23839900$

$-2.92418200$

$-2.41424600$

$-0.55619500$

$-3.14898200$

$-2.57555200$

$-0.52289100$

3.65678000

3.93904300

2. 32246200

4.60780700

4.50254600

4.71605300

2.77996900

2.70713400

0.39505700

0.60964500

0.08167000

0.45915200

$-0.79449000$

$-1.57878100$

$-3.22529800$

$-1.82800900$

$-3.01903800$

$-2.94347700$

$-3.79839600$

$-3.15984000$

$-2.74571700$

$-1.48837400$

1.24158000
Z

$-0.51870300$

0.11413000

0.80870800

0.88545900

0.28158200

$-0.46484600$

$-1.16715100$

$-1.14829200$

$-0.43418200$

0.34117100

1.15041600

0.76818300

$-0.63549600$

0.93448600

$-0.88245900$

0.14801800

$-0.19583800$

$-1.18774300$

0.99029000

$-1.96011300$

1.83953500

$-1.07288100$

0.05065800

1. 25736900

1. 38882100

$-1.72144900$

$-0.45929000$

1. 49219200

0.89054200

1.89492800

0.13772200

$-1.37780600$

$-0.78233100$

$-0.89333900$

$-1.88078200$

1.12173500

0.27889700

$-1.89889400$

$-0.71110500$

0.65577100

1.60744500

$-2.70724100$

$-2.47119200$

$-1.30786600$

2.61831300

1.21503000

2.34287400

1.73102800 


\section{CQ-MD1- Structure}

$\omega B 97 x-D / 6-31 G(d, p)$ - Water fully optimized XYZ coordinates

\section{Atom}

C

C

$\mathrm{C}$

$\mathrm{C}$

$\mathrm{C}$

C

$\mathrm{N}$

C

C

C

$\mathrm{N}$

C

$\mathrm{C}$

$\mathrm{C}$

$\mathrm{C}$

C

$\mathrm{N}$

C

C

C

C

$\mathrm{H}$

$\mathrm{Cl}$

$\mathrm{H}$

$\mathrm{H}$

$\mathrm{H}$

$\mathrm{H}$

$\mathrm{H}$

$\mathrm{H}$

$\mathrm{H}$

$\mathrm{H}$

$\mathrm{H}$

$\mathrm{H}$

$\mathrm{H}$

$\mathrm{H}$

$\mathrm{H}$

$\mathrm{H}$

$\mathrm{H}$

$\mathrm{H}$

$\mathrm{H}$

$\mathrm{H}$

$\mathrm{H}$

$\mathrm{H}$

$\mathrm{H}$

$\mathrm{H}$

$\mathrm{H}$

$\mathrm{H}$

$\mathrm{H}$

\section{$\mathrm{x}$}

3.92762100

4.26449300

3.45402200

2.30809400

1.92020200

2.73740400

2.45189200

1.32567000

0.43923500

0.72623400

$-0.03351400$

$-1.28729400$

$-2.45706900$

$-1.19033100$

$-2.63149800$

$-3.78451300$

$-4.02198000$

$-4.51844600$

$-2.85934000$

$-5.89867500$

$-2.28963700$

4.54104800

5.73561400

3.73569500

1.70143200

1.08328000

$-0.44538700$

$-1.44902900$

$-2.12755100$

$-0.37829000$

$-1.00639200$

$-2.30198100$

$-3.37288600$

$-1.69722200$

$-2.80974000$

$-3.60272600$

$-4.71038700$

$-3.81013200$

$-4.57441200$

$-3.18239700$

$-2.05870000$

$-6.26932700$

$-5.88679300$

$-6.60186000$

$-1.54448900$

$-3.07957900$

$-1.79977500$

0.25033700

\section{Y}

$-0.41803500$

$-0.86304100$

$-0.60167100$

0.13382700

0.62698900

0.33115500

0.71969900

1.38745300

1.74747200

1.41267900

1.79291300

2.53509000

1.63933200

3.76959600

0.41110200

$-0.46333100$

$-1.72098700$

$-1.53823800$

$-2.60590600$

$-0.89496200$

$-2.88530900$

$-0.62719900$

$-1.78728800$

$-0.97639300$

0.32157100

1.68363500

2.30802200

2.85749400

4.32867200

4.42139700

3.47860400

1.32346100

2.24119000

$-0.16256600$

0.72275400

$-0.68989100$

0.11936700

$-0.95937600$

$-2.53538800$

$-3.54744000$

$-2.21085800$

$-0.92082000$

0.14911200

$-1.43901700$

$-3.68111700$

$-3.19914600$

$-2.00419300$

1.49543900
Z

0.99463400

$-0.25353700$

$-1.37440100$

$-1.20752300$

0.06043700

1. 18415600

2.45935200

2. 61302000

1.59399700

0.27347200

$-0.77871100$

$-0.73741800$

$-1.14957800$

$-1.62733000$

$-0.26588100$

$-0.77390500$

$-0.06949500$

1. 29213800

$-0.05569500$

1.35804700

$-1.43872800$

1.86126300

$-0.47978400$

$-2.34908500$

$-2.08527200$

3. 63042400

1.85551500

0.29168200

$-1.59500100$

$-1.30157100$

$-2.66581900$

$-2.18983400$

$-1.14110600$

$-0.26537400$

0.76997300

$-1.83037800$

$-0.75414400$

1. 91324400

1. 74560000

0.40002400

0.59383000

2.38494400

1.03759500

0.72352200

$-1.38205700$

$-2.12534600$

$-1.86104500$

$-1.69869500$ 


\section{CQ-MD2- Structure}

$\omega B 97 x-D / 6-31 G(d, p)$ - Water fully optimized XYZ coordinates

\begin{tabular}{|c|c|c|c|}
\hline Atom & $\mathrm{x}$ & $\mathbf{Y}$ & $\mathbf{z}$ \\
\hline $\mathrm{C}$ & 3.38680500 & -0.84840700 & 0.69847400 \\
\hline C & 3.92330200 & -0.53453300 & -0.51885400 \\
\hline $\mathrm{C}$ & 3.30005000 & 0.37668700 & -1.39219800 \\
\hline $\mathrm{C}$ & 2.13907600 & 0.98671500 & -0.99166500 \\
\hline $\mathrm{C}$ & 1.56098600 & 0.72308100 & 0.27312200 \\
\hline $\mathrm{C}$ & 2.17879300 & -0.23743000 & 1.11911000 \\
\hline $\mathrm{N}$ & 1.67657900 & -0.62555400 & 2.32585800 \\
\hline C & 0.55321600 & -0.04104300 & 2.69321100 \\
\hline $\mathrm{C}$ & -0.13235500 & 0.94231600 & 1.97221700 \\
\hline $\mathrm{C}$ & 0.35961600 & 1.36838000 & 0.74315800 \\
\hline $\mathrm{N}$ & -0.22106300 & 2.35454100 & 0.01132400 \\
\hline $\mathrm{C}$ & -1.62618700 & 2.74438100 & 0.05499300 \\
\hline $\mathrm{C}$ & -2.38228600 & 2.16394700 & -1.15262300 \\
\hline $\mathrm{C}$ & -1.74445400 & 4.26287200 & 0.09736800 \\
\hline C & -2.16869900 & 0.66874000 & -1.37791000 \\
\hline $\mathrm{C}$ & -2.65923200 & -0.19508600 & -0.21801200 \\
\hline $\mathrm{N}$ & -2.25669300 & -1.58366900 & -0.35657000 \\
\hline $\mathrm{C}$ & -2.84257700 & -2.28458700 & -1.49494300 \\
\hline $\mathrm{C}$ & -2.25101300 & -2.31489100 & 0.90293800 \\
\hline C & -4.35127000 & -2.12890000 & -1.72175100 \\
\hline $\mathrm{C}$ & -3.60288900 & -2.54621500 & 1.58724200 \\
\hline $\mathrm{H}$ & 3.85727900 & -1.56087800 & 1.36311700 \\
\hline $\mathrm{Cl}$ & 5.41851800 & -1.28735000 & -1.02809400 \\
\hline $\mathrm{H}$ & 3.73387000 & 0.58003800 & -2.36170800 \\
\hline $\mathrm{H}$ & 1.66514700 & 1.67174300 & -1.68429000 \\
\hline $\mathrm{H}$ & 0.14204200 & -0.34777800 & 3.65159200 \\
\hline $\mathrm{H}$ & -1.02213600 & 1.38013200 & 2.40450300 \\
\hline $\mathrm{H}$ & -2.05291300 & 2.33604200 & 0.97189600 \\
\hline $\mathrm{H}$ & -2.79250400 & 4.55946000 & 0.07351300 \\
\hline $\mathrm{H}$ & -1.28515900 & 4.66730400 & 0.99949000 \\
\hline $\mathrm{H}$ & -1.24954500 & 4.70324600 & -0.77067800 \\
\hline $\mathrm{H}$ & -2.06267900 & 2.71108300 & -2.04701900 \\
\hline $\mathrm{H}$ & -3.44724700 & 2.38022500 & -1.01665000 \\
\hline $\mathrm{H}$ & -2.68059500 & 0.37899500 & -2.30174700 \\
\hline $\mathrm{H}$ & -1.10157100 & 0.46725000 & -1.53101700 \\
\hline $\mathrm{H}$ & -3.75165800 & -0.06239900 & -0.10843100 \\
\hline $\mathrm{H}$ & -2.21527000 & 0.16416200 & 0.71472100 \\
\hline $\mathrm{H}$ & -2.60405200 & -3.34343200 & -1.37888300 \\
\hline $\mathrm{H}$ & -2.33263100 & -1.95068100 & -2.40348000 \\
\hline $\mathrm{H}$ & -1.59707700 & -1.76021900 & 1.58848600 \\
\hline $\mathrm{H}$ & -1.76935400 & -3.28194500 & 0.72443300 \\
\hline $\mathrm{H}$ & -4.65350500 & -2.71185000 & -2.59552500 \\
\hline $\mathrm{H}$ & -4.93303600 & -2.47163500 & -0.86454900 \\
\hline $\mathrm{H}$ & -3.45563700 & -3.01300200 & 2.56539000 \\
\hline $\mathrm{H}$ & -4.13297700 & -1.60147800 & 1.73901000 \\
\hline $\mathrm{H}$ & -4.24265200 & -3.20409300 & 0.99422300 \\
\hline $\mathrm{H}$ & 0.24805900 & 2.62619200 & -0.83960200 \\
\hline $\mathrm{H}$ & -4.61606200 & -1.08491500 & -1.91674400 \\
\hline
\end{tabular}




\section{HYDROXYCHLOROQUINE}

\section{HCQ-M1-Structure}

WB97x-D/6-31G(d,p)- Water fully optimized XYZ coordinates

Atom

C

C

C

C

C

C

$\mathrm{N}$

C

C

$\mathrm{C}$

$\mathrm{N}$

C

C

C

C

C

$\mathrm{N}$

C

C

C

C

$\mathrm{H}$

$\mathrm{Cl}$

$\mathrm{H}$

$\mathrm{H}$

$\mathrm{H}$

$\mathrm{H}$

$\mathrm{H}$

$\mathrm{H}$

$\mathrm{H}$

$\mathrm{H}$

$\mathrm{H}$

$\mathrm{H}$

$\mathrm{H}$

$\mathrm{H}$

$\mathrm{H}$

$\mathrm{H}$

$\mathrm{H}$

$\mathrm{H}$

$\mathrm{H}$

$\mathrm{H}$

$\mathrm{H}$

$\mathrm{H}$

$\mathrm{H}$

$\mathrm{H}$

$\mathrm{H}$

$\mathrm{H}$

O

$\mathrm{H}$ $\mathrm{x}$

3.71412000

4.52925900

4.15940900

2.93382500

2. 03656200

2.45450300

1. 72143500

0.58438200

0.05907100

0.75323500

0.25125400

$-0.02772800$

$-0.83941300$

$-0.71159500$

$-2.21197600$

$-2.88310600$

$-3.79148900$

$-3.04960000$

$-4.82950000$

$-3.55931700$

$-5.89230900$

4.00547000

6.08361100

4.84711200

2.66674900

0.00636000

$-0.91975900$

0.92495700

$-0.95266300$

$-0.06481900$

$-1.64491800$

$-0.93188300$

$-0.25591300$

$-2.87539600$

$-2.11600100$

$-3.44307700$

$-2.11106700$

$-3.08142900$

$-1.99239000$

$-4.39201100$

$-5.31514500$

$-3.07915000$

$-4.64433700$

$-6.66643700$

$-5.48088600$

$-6.35851100$

$-0.57874200$

$-3.24868500$

$-3.56536700$
$\mathbf{Y}$

$-1.39533200$

$-0.32931300$

0.98783800

1.20760300

0.14102500

$-1.18547600$

$-2.29502400$

$-2.08724700$

$-0.82604300$

0.31709000

1.58367700

2.53173100

1.88524000

3.74739200

1. 37458700

0.49854300

$-0.46105700$

$-1.56817400$

$-0.95499900$

$-1.93004500$

0.08478500

$-2.41146300$

$-0.58913500$

1.80490600

2. 21090300

$-2.97295700$

$-0.76121600$

2.85261700

4.47698000

4.21948900

3.45869000

2.60974700

1.04383800

2.20744400

0.78162900

1.12878600

$-0.01877200$

$-2.46381200$

$-1.29980900$

$-1.35577600$

$-1.79923200$

$-2.86207700$

$-2.10501400$

$-0.35155900$

0.94576500

0.44611300

1. 49994900

$-0.86817100$

$-1.11290600$ z

0.33382900

0.59477300

0.25363300

$-0.31799700$

$-0.57401600$

$-0.28101400$

$-0.58287800$

$-1.21153600$

$-1.53868200$

$-1.18687200$

$-1.47011300$

$-0.36657800$

0.76564700

$-0.97766500$

0.34093000

1. 40381900

0.78292600

0.17719000

1. 68162100

$-1.20375700$

2.00359000

0.56511600

1. 35775200

0.42856200

$-0.62276200$

$-1.46344800$

$-2.00744400$

0.05893400

$-0.20332000$

$-1.71899400$

$-1.47079500$

1. 58164400

1.16000800

0.08688900

$-0.57355600$

2. 09903300

2.00373700

0.81660500

0.08112500

2.61464400

1.18112500

$-1.52936900$

$-1.15724700$

2. 63813400

2.53541200

1.08446800

$-2.04586600$

$-2.09342400$

$-2.96804700$ 


\begin{tabular}{|c|c|c|c|}
\hline Atom & $\mathrm{x}$ & $\mathbf{Y}$ & $\mathbf{z}$ \\
\hline $\mathrm{C}$ & -5.36286600 & 0.19314500 & 0.06287500 \\
\hline $\mathrm{C}$ & -5.27642800 & -1.16362700 & 0.21329500 \\
\hline $\mathrm{C}$ & -4.04000400 & -1.83587700 & 0.17205100 \\
\hline $\mathrm{C}$ & -2.89322800 & -1.10241200 & -0.00008500 \\
\hline $\mathrm{C}$ & -2.92807000 & 0.30560400 & -0.13757000 \\
\hline $\mathrm{C}$ & -4.18679500 & 0.96241000 & -0.12248000 \\
\hline $\mathrm{N}$ & -4.34612300 & 2.30774500 & -0.28405300 \\
\hline C & -3.23910000 & 2.99932500 & -0.46825200 \\
\hline C & -1.93926900 & 2.47722100 & -0.48423100 \\
\hline $\mathrm{C}$ & -1.74405300 & 1.11191500 & -0.30616300 \\
\hline $\mathrm{N}$ & -0.51766700 & 0.53035900 & -0.32111100 \\
\hline $\mathrm{C}$ & 0.73828700 & 1.27118800 & -0.35470400 \\
\hline C & 1.86683000 & 0.35294100 & -0.82805200 \\
\hline C & 1.04631400 & 1.93091800 & 0.99069400 \\
\hline C & 2.11043500 & -0.88672300 & 0.03144800 \\
\hline $\mathrm{C}$ & 3.34147700 & -1.66173400 & -0.43776300 \\
\hline $\mathrm{N}$ & 4.57330400 & -0.87575000 & -0.39186100 \\
\hline $\mathrm{C}$ & 5.02783500 & -0.69927900 & 0.97922700 \\
\hline C & 5.62178300 & -1.41298100 & -1.26625100 \\
\hline $\mathrm{C}$ & 6.18155700 & 0.28921200 & 1.13141600 \\
\hline $\mathrm{C}$ & 6.30877700 & -0.32787900 & -2.08956400 \\
\hline $\mathrm{H}$ & -6.31255600 & 0.71136700 & 0.08059400 \\
\hline $\mathrm{Cl}$ & -6.73498000 & -2.10001100 & 0.45867700 \\
\hline $\mathrm{H}$ & -4.00281700 & -2.91216300 & 0.27611300 \\
\hline $\mathrm{H}$ & -1.95336000 & -1.63996000 & -0.05032200 \\
\hline $\mathrm{H}$ & -3.36374800 & 4.07106900 & -0.60779500 \\
\hline $\mathrm{H}$ & -1.10467600 & 3.15160700 & -0.61659400 \\
\hline $\mathrm{H}$ & 0.62756200 & 2.05376600 & -1.11222600 \\
\hline $\mathrm{H}$ & 1.99715000 & 2.46596700 & 0.93627300 \\
\hline $\mathrm{H}$ & 0.26374600 & 2.64036500 & 1.26647200 \\
\hline $\mathrm{H}$ & 1.11933400 & 1.18169400 & 1.78205000 \\
\hline $\mathrm{H}$ & 2.79075600 & 0.93795300 & -0.85354300 \\
\hline $\mathrm{H}$ & 1.66278100 & 0.03432300 & -1.85603300 \\
\hline $\mathrm{H}$ & 2.20641500 & -0.61182100 & 1.08554100 \\
\hline $\mathrm{H}$ & 1.26128700 & -1.57531400 & -0.02602800 \\
\hline $\mathrm{H}$ & 3.17083900 & -1.94802700 & -1.48017600 \\
\hline $\mathrm{H}$ & 3.43525600 & -2.59667400 & 0.14335000 \\
\hline $\mathrm{H}$ & 5.33015700 & -1.66157300 & 1.43383400 \\
\hline $\mathrm{H}$ & 4.19216500 & -0.31406000 & 1.57229800 \\
\hline $\mathrm{H}$ & 5.16973000 & -2.13474300 & -1.95220000 \\
\hline $\mathrm{H}$ & 6.36379500 & -1.97794300 & -0.67954300 \\
\hline $\mathrm{H}$ & 7.08704400 & -0.09241900 & 0.65378000 \\
\hline $\mathrm{H}$ & 5.92102500 & 1.23720800 & 0.63916200 \\
\hline $\mathrm{H}$ & 7.05871800 & -0.76629800 & -2.75251000 \\
\hline $\mathrm{H}$ & 5.57239900 & 0.20197500 & -2.69849400 \\
\hline $\mathrm{H}$ & 6.80690000 & 0.40872300 & -1.45486900 \\
\hline $\mathrm{H}$ & -0.45344600 & -0.39978900 & 0.06026600 \\
\hline 0 & 6.51559500 & 0.47500400 & 2.49398100 \\
\hline $\mathrm{H}$ & 5.75595700 & 0.87823600 & 2.92506600 \\
\hline
\end{tabular}


Atom

$\mathrm{C}$

$\mathrm{C}$

C

C

C

C

$\mathrm{N}$

C

C

C

$\mathrm{N}$

C

C

C

C

C

$\mathrm{N}$

C

C

C

C

$\mathrm{H}$

CI

$\mathrm{H}$

$\mathrm{H}$

$\mathrm{H}$

$\mathrm{H}$

$\mathrm{H}$

$\mathrm{H}$

$\mathrm{H}$

$\mathrm{H}$

$\mathrm{H}$

$\mathrm{H}$

$\mathrm{H}$

$\mathrm{H}$

$\mathrm{H}$

$\mathrm{H}$

$\mathrm{H}$

$\mathrm{H}$

$\mathrm{H}$

$\mathrm{H}$

$\mathrm{H}$

$\mathrm{H}$

$\mathrm{H}$

$\mathrm{H}$

$\mathrm{H}$

$\mathrm{H}$

O

$\mathrm{H}$ $\mathrm{x}$

$$
\begin{aligned}
& -4.80650300 \\
& -5.28970500 \\
& -4.48896900 \\
& -3.19072300 \\
& -2.64178000 \\
& -3.46724600 \\
& -3.05894200 \\
& -1.80955100 \\
& -0.89451800
\end{aligned}
$$

$-1.28163700$

$-0.45587300$

0.88996300

1.97546300

1.05503100

1.94359300

3.03273700

4.37894900

5.05337400

5.20306200

6.02962700

4.73599600

$-5.42193300$

$-6.94724900$

$-4.89772300$

$-2.59357900$

$-1.47461400$

0.11157700

0.97302500

2.04441900

0.29997300

0.96161200

1.86803600

2.95413900

0.96489000

2.07953700

2.91427300

2.88882400

5.59013000

4.30034900

5.22849200

6.23157600

6.61265500

6.73846100

5.34912000

3.69767000

4.79940000

$-0.80130000$

5.30157200

5.93343600
Y

0.95227300

$-0.10242600$

$-1.21829000$

$-1.24101100$

$-0.17472300$

0.94196600

2.02358500

1.98984500

0.95805200

$-0.16516700$

$-1.20753600$

$-1.39998800$

$-0.97179700$

$-2.86546800$

0.50537600

0.88137900

0.66225400

$-0.48987800$

1.86372400

$-1.15543600$

2.90413200

1.81015700

$-0.07829000$

$-2.04055800$

$-2.11179800$

2.85289000

1.06168100

$-0.79343400$

$-3.02981200$

$-3.16725200$

$-3.50121900$

$-1.58026200$

$-1.21685700$

0.76271800

1.11600700

1.93470500

0.31018700

$-0.22556600$

$-1.23556600$

2.30316600

1.57900200

$-1.89560800$

$-0.41496500$

3.80456700

3.19273900

2. 50119700

$-1.96609400$

$-1.77042900$

$-2.17916300$
Z

0.10625700

$-0.61686200$

$-0.92475500$

$-0.48085100$

0.26968100

0.57176500

1.29411800

1.71574100

1.48679600

0.75863800

0.51244800

1. 04563200

0.05596700

1. 43224000

$-0.31811600$

$-1.31868400$

$-0.78454700$

$-1.36768100$

$-0.81741600$

$-0.41547800$

0.18943000

0.34335300

$-1.17890700$

$-1.49649300$

$-0.72325100$

2.28587900

1.86746400

1.95149500

1. 86128300

2.15915800

0.54660000

$-0.85263700$

0.48308900

$-0.73706800$

0.58294600

$-1.59186400$

$-2.24616300$

$-2.29530900$

$-1.63595400$

$-1.83113800$

$-0.57887600$

$-0.97919800$

$-0.02110400$

0.12935900

0.00816500

1. 20125100

$-0.05246900$

0.63096100

1. 22725100 


\section{HCQ-M4-Structure}

wB97x-D/6-31G(d,p) - Water fully optimized XYZ coordinates

\section{Atom}

C

C

C

C

C

C

$\mathrm{N}$

C

C

C

$\mathrm{N}$

C

C

C

C

C

$\mathrm{N}$

C

C

C

C

$\mathrm{H}$

Cl

$\mathrm{H}$

$\mathrm{H}$

$\mathrm{H}$

$\mathrm{H}$

$\mathrm{H}$

$\mathrm{H}$

$\mathrm{H}$

$\mathrm{H}$

$\mathrm{H}$

$\mathrm{H}$

$\mathrm{H}$

$\mathrm{H}$

$\mathrm{H}$

$\mathrm{H}$

$\mathrm{H}$

$\mathrm{H}$

$\mathrm{H}$

$\mathrm{H}$

$\mathrm{H}$

$\mathrm{H}$

$\mathrm{H}$

$\mathrm{H}$

$\mathrm{H}$

$\mathrm{H}$

O

$\mathrm{H}$ $\mathrm{x}$

2.54061800

3.03454100

2.27323300

0.98260600

0.39725500

1.21090700

0.80033600

$-0.43244100$

$-1.34008700$

$-0.96660900$

$-1.88222500$

$-2.12369300$

$-1.98591300$

$-3.50406400$

$-2.92559700$

$-2.54717900$

$-1.25378200$

$-0.18528300$

$-1.34809800$

1.11069300

$-2.07331500$

3.13195600

4.64486400

2.70713900

0.40734200

$-0.76228400$

$-2.35386400$

$-1.39961300$

$-3.70019600$

$-3.56775200$

$-4.28262100$

$-2.15289800$

$-0.95566700$

$-3.96185500$

$-2.86725700$

$-3.32689900$

$-2.51882500$

$-0.04250300$

$-0.43044000$

$-1.84244300$

$-0.32929600$

1.34520300

0.95230200

$-2.08952600$

$-3.10938300$

$-1.57496000$

$-2.75667500$

2.15094000

2.95504900
Y

$-0.52748500$

$-0.77530600$

$-1.44219200$

$-1.79648100$

$-1.50606800$

$-0.90368800$

$-0.66104600$

$-1.02716600$

$-1.58033800$

$-1.80371800$

$-2.33031800$

$-1.84938700$

$-0.32981500$

$-2.34524800$

0.49744700

1.97969800

2.22193100

2.70285000

2.96522600

1.98697600

2.18754800

$-0.03643600$

$-0.22903500$

$-1.66045200$

$-2.31937400$

$-0.84806600$

$-1.79080000$

$-2.31720200$

$-2.06940200$

$-3.43127800$

$-1.89822600$

$-0.07818800$

$-0.04139500$

0.37058300

0.15947500

2.57703600

2.30958300

3.79333300

2. 47851200

3.94162800

3.17840700

2.12546100

0.91450100

2.75670200

1.97761100

1.23214600

$-2.52730500$

2.48420700

2.01200400
Z

1.10508400

$-0.14343400$

$-1.12451200$

$-0.83179600$

0.42705800

1.42836800

2.70305600

2.99348700

2.08455700

0.76668500

$-0.11739200$

$-1.49212500$

$-1.63318800$

$-1.90702000$

$-0.75913300$

$-0.80127200$

$-0.16377000$

$-1.02697500$

1.08479800

$-0.68080300$

2.17278700

1.86651500

$-0.56094800$

$-2.09079100$

$-1.58238900$

4.01296900

2.40423200

$-2.16541200$

$-2.94413400$

$-1.81650400$

$-1.28164000$

$-2.68673200$

$-1.40450200$

$-1.08897500$

0.28201200

$-0.31107100$

$-1.84673400$

$-0.95365600$

$-2.06856300$

0.93038200

1. 42321700

0.38449200

$-0.83993800$

3.10364400

1.89317900

2.34826400

0.35060200

$-1.50532000$

$-1.26844800$ 


\section{HCQ-M5-Structure}

wB97x-D/6-31G(d,p) - Water fully optimized XYZ coordinates

\section{Atom}

C

C

C

C

C

C

$\mathrm{N}$

C

C

C

$\mathrm{N}$

C

C

C

C

C

$\mathrm{N}$

C

C

C

C

$\mathrm{H}$

$\mathrm{Cl}$

$\mathrm{H}$

$\mathrm{H}$

$\mathrm{H}$

$\mathrm{H}$

$\mathrm{H}$

$\mathrm{H}$

$\mathrm{H}$

$\mathrm{H}$

$\mathrm{H}$

$\mathrm{H}$

$\mathrm{H}$

$\mathrm{H}$

$\mathrm{H}$

$\mathrm{H}$

$\mathrm{H}$

$\mathrm{H}$

$\mathrm{H}$

$\mathrm{H}$

$\mathrm{H}$

$\mathrm{H}$

$\mathrm{H}$

$\mathrm{H}$

$\mathrm{H}$

$\mathrm{H}$

O

$\mathrm{H}$
$\mathrm{X}$

$-2.96683800$

$-3.03492300$

$-2.42690300$

$-1.72347800$

$-1.58872300$

$-2.25142700$

$-2.25024000$

$-1.61340300$

$-0.89983700$

$-0.82962800$

$-0.06703100$

0.95993000

1. 56574500

2.02333300

2. 20972300

1.87472600

2.05356800

3. 43824300

1. 48379000

3. 51787700

$-0.03890900$

$-3.44864500$

$-3.92597600$

$-2.52681200$

$-1.27505000$

$-1.62610700$

$-0.35225100$

0.50033700

2. 74930600

1.57673800

2.55917200

2.30204300

0.80112300

3. 29021700

1.84281800

2.45173000

0.81786600

4. 00863400

3. 93221600

1.85377600

1.84751800

3. 04052200

2. 95982700

$-0.40021000$

$-0.47708900$

$-0.41387400$

0.30134100

4.85295500

5. 25295400
$\mathbf{Y}$

$-0.78029200$

$-1.17371500$

$-0.43073400$

0.69930200

1. 12997500

0.39517800

0.75237900

1. 86572800

2. 65470800

2.28094700

3. 01247700

2.39677100

1. 12320700

3. 45427400

1. 32600100

0.18434200

$-1.13608800$

$-1.42150200$

$-2.18456600$

$-2.38153000$

$-2.20686900$

$-1.33882300$

$-2.61817400$

$-0.75147900$

1. 28119600

2.16024500

3. 52000600

2.13858600

3. 07527500

4. 36436800

3. 70829000

0.72710600

0.34766400

1. 45783700

2. 24785200

0.29468400

0.28088100

$-1.82511900$

$-0.49279000$

$-2.09371200$

$-3.14659000$

$-3.33421900$

$-1.93882700$

$-3.08475900$

$-1.32466600$

$-2.25521800$

3. 84187500

$-2.68373000$

$-1.86959000$
Z

0.50449300

$-0.80129900$

$-1.83456000$

$-1.51265400$

$-0.16942400$

0.85122800

2.16516200

2.46707700

1. 55427000

0.22277200

$-0.67193200$

$-1.54634700$

$-0.94720100$

$-1.81646200$

0.43226400

1.39045100

0.79992400

0.45368200

1.64350400

$-0.72974100$

1.63177300

1.29621800

$-1.22619400$

$-2.86261600$

$-2.30659300$

3. 51248100

1. 90860600

$-2.50478000$

$-2.53728100$

$-2.22149400$

$-0.89661900$

$-1.65441200$

$-0.86769300$

0.33025400

0.89599200

2.32572100

1. 65798400

1. 30668000

0.15407700

2.68110700

1.27211500

$-0.48639300$

$-1.56556900$

2.17272200

2.10519900

0.60547400

$-0.22415000$

$-1.08287900$

$-1.40371600$ 


\section{HCQ-M6-Structure}

wB97x-D/6-31G(d,p) - Water fully optimized XYZ coordinates

\section{Atom}

C

C

C

C

C

C

$\mathrm{N}$

C

C

C

$\mathrm{N}$

C

C

C

C

C

$\mathrm{N}$

C

C

C

C

$\mathrm{H}$

Cl

$\mathrm{H}$

$\mathrm{H}$

$\mathrm{H}$

$\mathrm{H}$

$\mathrm{H}$

$\mathrm{H}$

$\mathrm{H}$

$\mathrm{H}$

$\mathrm{H}$

$\mathrm{H}$

$\mathrm{H}$

$\mathrm{H}$

$\mathrm{H}$

$\mathrm{H}$

$\mathrm{H}$

$\mathrm{H}$

$\mathrm{H}$

$\mathrm{H}$

$\mathrm{H}$

$\mathrm{H}$

$\mathrm{H}$

$\mathrm{H}$

$\mathrm{H}$

$\mathrm{H}$

O

$\mathrm{H}$

\section{$\mathrm{x}$}

$-2.71983700$

$-3.38893500$

$-3.16922500$

$-2.22704100$

$-1.47410100$

$-1.76592200$

$-1.17252100$

$-0.31402500$

0.07355600

$-0.44001400$

0.04008000

0.43218700

1. 05618100

1. 37551800

2.23492000

2.75391900

2.83010800

1. 49296800

3. 67625800

1. 45378000

5.16198500

$-2.90347900$

$-4.56840700$

$-3.74351900$

$-2.07550800$

0.14777600

0.83813200

$-0.45311700$

1. 61880300

0.91106600

2. 30838200

1. 37038100

0.28887900

3. 04425400

1. 93376100

3. 75163700

2. 11319500

1. 09878200

0.80781800

3.40695700

3. 47517800

2. 21722400

1. 70166800

5.75050700

5.44597500

5.42774100

0.72795800

0.21307600

$-0.40884300$
Y

$-1.15979900$

$-0.58257400$

0.76048300

1. 48971500

0.92636500

$-0.40360100$

$-1.01498800$

$-0.29087800$

1.00963600

1.62696000

2.85396300

3. 19215100

2. 00972600

4.38615200

1. 33544500

0.12014700

$-1.09652600$

$-1.63418500$

$-2.09296300$

$-2.58383800$

$-1.79868500$

$-2.18246500$

$-1.51311700$

1. 20129500

2. 52526800

$-0.78359400$

1. 50138900

3. 51797600

4. 72153700

5.21357500

4. 12217200

2. 37812800

1. 25160100

2.05333100

0.99618100

0.33247200

$-0.05479600$

$-2.16036300$

$-0.80325000$

$-2.18535000$

$-3.06588900$

$-3.36032500$

$-2.01461500$

$-2.59395000$

$-0.85807000$

$-1.73665800$

3.19792600

$-3.24225200$

$-2.61121300$
Z

0.30205200

$-0.74027100$

$-1.11030200$

$-0.43582900$

0.62414800

1.02816200

2.09206900

2.78932900

2.46663300

1. 33215800

0.94296800

$-0.44266500$

$-1.19728400$

$-0.36903300$

$-0.49524100$

$-1.28066400$

$-0.46951500$

$-0.22220800$

$-1.13076300$

0.97621800

$-0.98468700$

0.60443800

$-1.63264100$

$-1.91402400$

$-0.70527800$

3.63969200

3.05567400

$-0.99530100$

$-1.37773000$

0.17049600

0.13666300

$-2.17948400$

$-1.39097500$

$-0.33995700$

0.49956800

$-1.66774300$

$-2.16576800$

$-1.10922400$

$-0.02027900$

$-2.19805500$

$-0.67860400$

0.86902500

1.88354200

$-1.44655100$

$-1.46197700$

0.07157400

1.60000600

1.09456500

1.50146600 


\section{Atom}

C

C

C

C

C

C

$\mathrm{N}$

C

C

C

$\mathrm{N}$

C

C

C

C

C

$\mathrm{N}$

C

C

C

C

$\mathrm{H}$

$\mathrm{Cl}$

$\mathrm{H}$

$\mathrm{H}$

$\mathrm{H}$

$\mathrm{H}$

$\mathrm{H}$

$\mathrm{H}$

$\mathrm{H}$

$\mathrm{H}$

$\mathrm{H}$

$\mathrm{H}$

$\mathrm{H}$

$\mathrm{H}$

$\mathrm{H}$

$\mathrm{H}$

$\mathrm{H}$

$\mathrm{H}$

$\mathrm{H}$

$\mathrm{H}$

$\mathrm{H}$

$\mathrm{H}$

$\mathrm{H}$

$\mathrm{H}$

$\mathrm{H}$

$\mathrm{H}$

O

$\mathrm{H}$

\section{$\mathrm{x}$}

$-3.71725200$

$-4.47588800$

$-4.04524400$

$-2.81883300$

$-1.98123600$

$-2.45590800$

$-1.77181900$

$-0.64351900$

$-0.08659000$

$-0.70496400$

$-0.12796500$

0.21758700

0.96623100

0.99790500

2.28711900

2.94264700

4.03402900

3.61830800

5.00320600

2.64695900

5.73621100

$-4.05810500$

$-6.03614500$

$-4.68803800$

$-2.50290400$

$-0.08115900$

0.86957700

$-0.71107900$

1.27417200

0.40042500

1.91839800

1.13676000

0.30545600

2.97530800

2.11178100

3.30062300

2. 17568600

4.51486200

3.14885100

5.72803800

4.54589300

3.06158400

1.70582000

6.51057700

6.20359700

5.05718000

0.69105700

2.40583600

1.91325800
Y

$-1.21144000$

$-0.11537900$

1.19292200

1.37023200

0.26961800

$-1.04467500$

$-2.17779200$

$-2.00671700$

$-0.75798900$

0.40440300

1.65105000

2.50780500

1.74452000

3.70251700

1.11334700

0.34808900

$-0.56523200$

$-1.93161900$

$-0.03324100$

$-2.12262800$

1.18075700

$-2.21792900$

$-0.32483500$

2.03641900

2. 36775200

$-2.90333500$

$-0.71923100$

2.87865700

4.36640100

4.26352300

3.37965100

2.43547000

0.95544500

1.88374500

0.43776600

1.08831300

$-0.24163700$

$-2.52969300$

$-2.33951600$

$-0.82642200$

0.22854900

$-1.68061700$

$-1.60584700$

1.50552800

0.93633800

2.02266300

1.54907300

$-3.48929800$

$-3.85378400$
Z

$-0.23670700$

$-0.54107000$

$-0.23949700$

0.34543200

0.65010800

0.38839300

0.71999300

1. 37438600

1.70063500

1.28725500

1.50874500

0.35073400

$-0.75304000$

0.88018500

$-0.32145400$

$-1.48418200$

$-1.15335100$

$-0.87775100$

$-0.20495200$

0.29027300

$-0.75982400$

$-0.43950900$

$-1.30605000$

$-0.45400700$

0.62065100

1.62580900

2. 21117100

$-0.08775600$

0.05951000

1.60011100

1.37539200

$-1.58641100$

$-1.13211900$

0.04286000

0.52088400

$-2.21051800$

$-1.99820900$

$-0.68577300$

$-1.78226600$

$-0.00045400$

0.76377300

1.20172000

0.07127400

$-0.06258100$

$-1.71627100$

$-0.91748300$

2.09784200

0.57835800

$-0.16290100$ 


\section{HCQ-M8-Structure}

wB97x-D/6-31G(d,p) - Water fully optimized XYZ coordinates

\section{Atom}

C

C

C

C

C

C

$\mathrm{N}$

C

C

C

$\mathrm{N}$

C

C

C

C

C

$\mathrm{N}$

C

C

C

C

$\mathrm{H}$

Cl

$\mathrm{H}$

$\mathrm{H}$

$\mathrm{H}$

$\mathrm{H}$

$\mathrm{H}$

$\mathrm{H}$

$\mathrm{H}$

$\mathrm{H}$

$\mathrm{H}$

$\mathrm{H}$

$\mathrm{H}$

$\mathrm{H}$

$\mathrm{H}$

$\mathrm{H}$

$\mathrm{H}$

$\mathrm{H}$

$\mathrm{H}$

$\mathrm{H}$

$\mathrm{H}$

$\mathrm{H}$

$\mathrm{H}$

$\mathrm{H}$

$\mathrm{H}$

$\mathrm{H}$

O

$\mathrm{H}$ $\mathrm{x}$

$-5.22963300$

$-4.80214300$

$-3.43371800$

$-2.50797700$

$-2.89946200$

$-4.28392300$

$-4.77687300$

$-3.87800500$

$-2.48964700$

$-1.95779500$

$-0.62596100$

0.35824400

1. 56154600

0.75571600

2. 47516000

3. 46003200

4. 66631800

4.35596800

5.66526000

5.55194900

7.05433900

$-6.28100800$

$-5.97507300$

$-3.12702800$

$-1.45878300$

$-4.26537800$

$-1.85394500$

$-0.10441200$

1. 47401000

$-0.11376800$

1. 21472900

2. 15056700

1.19179700

3. 04407600

1.87849900

3. 77745500

2. 93295100

3.87771800

3. 63928100

5.35842800

5.69329100

5.19681500

6.29024300

7. 79887800

7. 05726100

7.34049500

$-0.34336600$

6.12002100

6.86742500
$Y$

$-0.43903700$

$-1.73547400$

$-2.06605400$

$-1.05499400$

0.30306800

0.61557100

1.88431000

2.84665600

2.67529500

1.39555300

1.16070400

2.21837100

1. 64883900

2.89041300

0.73169200

0.00978600

$-0.45274800$

$-1.42367900$

$-0.94942300$

$-1.88363100$

$-0.38622600$

$-0.18515800$

$-3.03142200$

$-3.10215900$

$-1.32513900$

3. 85868400

3.54855100

2. 96965100

3. 69052700

3. 31956500

2.16693600

2.49149800

1. 11179500

1.31691600

0.01172700

0.71106500

$-0.81631100$

$-2.32776300$

$-0.97339800$

$-0.66626200$

$-2.04905800$

$-2.63549500$

$-2.37383400$

$-0.83575900$

0.69395300

$-0.56179700$

0.23445900

$-0.77168200$

$-1.09177800$
Z

0.03601900

$-0.04368200$

$-0.05758700$

$-0.01447700$

0.04725500

0.09233600

0.18860600

0.24091300

0.17993400

0.05851100

$-0.04895700$

$-0.26723000$

$-1.02210600$

1.04545700

$-0.20640300$

$-1.12451800$

$-0.44966300$

0.59124400

$-1.40266400$

1.40414200

$-1.12496500$

0.05741100

$-0.13783100$

$-0.10417900$

$-0.01197400$

0.32911800

0.21171900

$-0.91855500$

0.85355400

1.54629000

1.72180800

$-1.39536200$

$-1.90351200$

0.52298800

0.36584700

$-1.90350800$

$-1.64024300$

0.16772900

1.28342600

$-2.41328600$

$-1.39670000$

2. 12031000

0.75554800

$-1.78618800$

$-1.28878300$

$-0.08645600$

$-0.32604300$

2.07200300

2. 58317100 
Atom

C

C

C

C

C

C

$\mathrm{N}$

C

C

C

$\mathrm{N}$

C

C

C

C

C

$\mathrm{N}$

C

C

C

C

$\mathrm{H}$

$\mathrm{Cl}$

$\mathrm{H}$

$\mathrm{H}$

$\mathrm{H}$

$\mathrm{H}$

$\mathrm{H}$

$\mathrm{H}$

$\mathrm{H}$

$\mathrm{H}$

$\mathrm{H}$

$\mathrm{H}$

$\mathrm{H}$

$\mathrm{H}$

$\mathrm{H}$

$\mathrm{H}$

$\mathrm{H}$

$\mathrm{H}$

$\mathrm{H}$

$\mathrm{H}$

$\mathrm{H}$

$\mathrm{H}$

$\mathrm{H}$

$\mathrm{H}$

$\mathrm{H}$

$\mathrm{H}$

O

$\mathrm{H}$ $\mathrm{x}$

$-5.16625300$

$-5.14419800$

$-3.95493500$

$-2.78486700$

$-2.75125500$

$-3.96637000$

$-4.06002800$

$-2.92629800$

$-1.66201800$

$-1.53429300$

$-0.33952200$

0.94990700

2. 00005500

1.37041300

2.44743100

3. 53468800

4.67929100

5.50288000

5.47339700

5.88337800

4.79685500

$-6.07916200$

$-6.62384900$

$-3.97070700$

$-1.87883200$

$-2.99580500$

$-0.80231500$

0.83307100

2.40662100

0.72482900

1. 30427800

2.88797300

1.61807500

2.83047000

1.61289000

3. 11390000

3.83391800

6.42036200

4. 95709800

5.73562200

6.41989900

6.67065900

6.29221200

5.44684700

3.85625400

4.57971600

$-0.31280600$

4. 72815400

4. 90584100
$\mathbf{Y}$

0.19035200

$-1.09729300$

$-1.70842100$

$-0.99538400$

0.33332000

0.94106800

2. 21552500

2. 88100100

2.39549200

1.10035600

0.56102400

1. 17360800

0.08510500

2.18511600

$-0.66354700$

$-1.68726600$

$-1.07177300$

$-0.27327200$

$-2.00364800$

1.06432300

$-2.39267500$

0.66344900

$-2.03431400$

$-2.72457400$

$-1.48324600$

3. 89439800

3.03725900

1.69734300

2. 48955100

3. 06734700

1. 74053800

0.55707600

$-0.63172600$

0.06344300

$-1.18433500$

$-2.43773800$

$-2.21901300$

$-0.80898500$

$-0.07602500$

$-2.91030000$

$-1.50532700$

1.52977400

0.90162500

$-3.04618700$

$-2.92365600$

$-1.49677100$

$-0.41737200$

1.88581100

2.61745700
Z

-0.29260800
0.16301700
0.59939400
0.53598800
0.05195600
-0.35904700
-0.83556700
-0.91289700
-0.55928200
-0.07028400
0.26397400

$-0.04665600$

$-0.27021500$

1.02088700

0.98349900

0.65915000

$-0.00082400$

0.90089600

$-0.79299800$

0.29392100

$-2.10075100$

$-0.62825300$

0.18956500

0.97112100

0.87679100

$-1.30285700$

$-0.67934700$

$-1.00407700$

0.84996300

1.00596000

2.01473500

$-0.69443100$

$-1.00659300$

1.71156600

1.47126300

$-0.01762800$

1. 57874500

1.18935800

1.82815300

$-0.21479300$

$-1.03103000$

0.90271400

$-0.71230200$

$-2.68624900$

$-1.93586300$

$-2.68730500$

0.49971900

0.25085100

$-0.34566100$ 


\section{HCQ-M10-Structure}

WB97x-D/6-31G(d,p) - Water fully optimized XYZ coordinates

\section{Atom}

C

C

C

C

C

C

$\mathrm{N}$

C

C

C

$\mathrm{N}$

C

C

C

C

C

$\mathrm{N}$

C

C

C

C

$\mathrm{H}$

Cl

$\mathrm{H}$

$\mathrm{H}$

$\mathrm{H}$

$\mathrm{H}$

$\mathrm{H}$

$\mathrm{H}$

$\mathrm{H}$

$\mathrm{H}$

$\mathrm{H}$

$\mathrm{H}$

$\mathrm{H}$

$\mathrm{H}$

$\mathrm{H}$

$\mathrm{H}$

$\mathrm{H}$

$\mathrm{H}$

$\mathrm{H}$

$\mathrm{H}$

$\mathrm{H}$

$\mathrm{H}$

$\mathrm{H}$

$\mathrm{H}$

$\mathrm{H}$

$\mathrm{H}$

O

$\mathrm{H}$ $\mathrm{x}$

2.76432500

2.95743700

2.08648200

0.99282100

0.72683400

1.65352200

1.52692200

0.50958000

$-0.46729400$

$-0.43635200$

$-1.49390300$

$-2.14616700$

$-2.08573600$

$-3.57991400$

$-2.53906100$

$-2.60694500$

$-1.40424900$

$-0.20928800$

$-1.09110400$

$-0.36330200$

$-2.26927000$

3.43985600

4.32612300

2.28518600

0.33157200

0.41939500

$-1.30956900$

$-1.65179800$

$-4.08322300$

$-3.59591600$

$-4.14298900$

$-2.70281500$

$-1.06318700$

$-3.53551800$

$-1.87292600$

$-2.91011700$

$-3.40792600$

0.58151300

0.16347200

$-0.64615300$

$-0.31131900$

$-0.87880600$

$-0.97655400$

$-1.91041300$

$-2.99034400$

$-2.78832000$

$-2.18669600$

0.89386700

1.26775700
Y

0.28691800

0.06158100

0.58904200

1.30671300

1.53953100

1.05633500

1.26158600

2.01538700

2.52990400

2.22620600

2.57687700

1.68687100

0.19446400

2.18033200

$-0.11872400$

$-1.60862000$

$-2.42722700$

$-1.86567300$

$-2.92905900$

$-1.50345200$

$-3.56384800$

$-0.10244900$

$-0.89029100$

0.42333600

1.72003800

2.19038800

3.06923700

1.80936800

1.61155500

3.23679500

2.05746900

$-0.35344900$

$-0.16155100$

0.30242700

0.38282600

$-1.66350700$

$-2.08718200$

$-2.62211800$

$-0.98477200$

$-2.15547500$

$-3.68717800$

$-2.32185600$

$-0.59869900$

$-4.10184400$

$-2.81782400$

$-4.27124800$

3.09078500

$-1.33983400$

$-0.49356100$
Z

$-0.31111200$

1.02269700

1.99868900

1.59310000

0.22142200

$-0.74133000$

$-2.08208600$

$-2.46752200$

$-1.61666400$

$-0.25940500$

0.54227800

1. 53189700

1.15636900

1.69196600

$-0.26804500$

$-0.63542100$

$-0.49263800$

$-1.10375600$

0.84408700

$-2.58123100$

1.56830700

$-1.06132600$

1.54866900

3.04946600

2. 34269400

$-3.53559200$

$-2.03214900$

2. 50046400

2.47441700

1.96588700

0.76229200

1.87818900

1.29656700

$-0.44222100$

$-0.97452000$

$-1.68709200$

$-0.06794600$

$-1.04903200$

$-0.55289000$

1.49288100

0.71004600

$-3.09226800$

$-2.70228000$

2.44736500

1.91083900

0.91674000

0.01421900

$-3.20360900$

$-2.90041700$ 


\section{Atom}

C

C

C

C

C

C

$\mathrm{N}$

C

C

C

$\mathrm{N}$

C

C

C

C

C

$\mathrm{N}$

C

C

C

C

$\mathrm{H}$

$\mathrm{Cl}$

$\mathrm{H}$

$\mathrm{H}$

$\mathrm{H}$

$\mathrm{H}$

$\mathrm{H}$

$\mathrm{H}$

$\mathrm{H}$

$\mathrm{H}$

$\mathrm{H}$

$\mathrm{H}$

$\mathrm{H}$

$\mathrm{H}$

$\mathrm{H}$

$\mathrm{H}$

$\mathrm{H}$

$\mathrm{H}$

$\mathrm{H}$

$\mathrm{H}$

$\mathrm{H}$

$\mathrm{H}$

$\mathrm{H}$

$\mathrm{H}$

$\mathrm{H}$

$\mathrm{H}$

O

$\mathrm{H}$ $\mathrm{x}$

$-4.45568900$

$-4.42591200$

$-3.28557100$

$-2.17290800$

$-2.14496800$

$-3.31405200$

$-3.41909700$

$-2.35038700$

$-1.13549800$

$-0.98772300$

0.17652000

1. 35627900

2. 11213300

2. 23042700

3.17689400

2. 66118400

3. 23640800

4. 55091000

2.38032100

5.62980700

1. 05057400

$-5.33656000$

$-5.84174200$

$-3.29509800$

$-1.31259400$

$-2.43469300$

$-0.32278700$

1. 00404100

3.12891900

1.69122200

2.55124900

2.60208400

1.37554800

3. 67451200

3. 94392300

2.83684200

1.57911300

4.46663500

4. 90891000

2.93598800

2.19738800

6.52266900

5. 33842000

0.55409600

1. 20950400

0.37169100

0.18695600

5.89443500

6.56440900
$\mathbf{Y}$

0.22816200

$-1.04592800$

$-1.56192800$

$-0.76779700$

0.55022900

1. 06192000

2. 32432600

3. 08524100

2. 70085800

1.41047300

0.96047700

1. 79264900

2.12126800

1. 10844600

1. 12357500

$-0.28555700$

$-1.35775400$

$-1.78566700$

$-2.54514400$

$-0.72578700$

$-2.34635600$

0.63167300

$-2.06543500$

$-2.56714800$

$-1.18479900$

4. 09564000

3. 41300200

2.74322800

1.70122600

0.99967700

0.12150400

3. 08883700

2.26947200

1.56571500

1.02289700

$-0.49081300$

$-0.30336600$

$-2.23858900$

$-2.57095000$

$-3.33010200$

$-2.92364000$

$-1.21217600$

0.06281800

$-3.30915000$

$-1.90213400$

$-1.71148000$

0.05371400

$-0.19945900$

0.48172900
Z

$-0.50032100$

$-0.00489900$

0.63787400

0.75284600

0.23816700

$-0.38685800$

$-0.89372100$

$-0.76499300$

$-0.18602300$

0.31551300

0.83901400

1.06368000

$-0.24223100$

2.10685300

$-0.70460100$

$-1.07072500$

$-0.24995100$

$-0.73041000$

$-0.22281400$

$-0.81929200$

0.48709800

$-0.98229000$

$-0.14485500$

1.03721100

1. 26268300

$-1.15752400$

$-0.15480600$

1. 48408800

2. 28701100

3. 05043500

1.75598500

$-0.09463300$

$-1.03806600$

$-1.57284900$

0.06709300

$-2.14120800$

$-0.93220000$

$-1.73766600$

$-0.05717500$

0.29852900

$-1.24462500$

$-1.23280100$

$-1.52308000$

0.62210300

1.47497600

$-0.08617800$

1. 27390800

0.46908000

0.37039900 


\section{HCQ-M12-Structure}

WB97x-D/6-31G(d,p) - Water fully optimized XYZ coordinates

\section{Atom}

C

C

C

C

C

C

$\mathrm{N}$

C

C

C

$\mathrm{N}$

C

C

C

C

C

$\mathrm{N}$

C

C

C

C

$\mathrm{H}$

Cl

$\mathrm{H}$

$\mathrm{H}$

$\mathrm{H}$

$\mathrm{H}$

$\mathrm{H}$

$\mathrm{H}$

$\mathrm{H}$

$\mathrm{H}$

$\mathrm{H}$

$\mathrm{H}$

$\mathrm{H}$

$\mathrm{H}$

$\mathrm{H}$

$\mathrm{H}$

$\mathrm{H}$

$\mathrm{H}$

$\mathrm{H}$

$\mathrm{H}$

$\mathrm{H}$

$\mathrm{H}$

$\mathrm{H}$

$\mathrm{H}$

$\mathrm{H}$

$\mathrm{H}$

O

$\mathrm{H}$ $\mathrm{x}$

4.80414200

4.96118600

3.94022400

2.75447400

2.54084400

3.58352800

3.48794600

2.33985500

1.23745700

1.30304400

0.26393200

$-1.00120800$

$-2.03292800$

$-0.87376400$

$-2.39460400$

$-3.16806900$

$-4.62235700$

$-5.27920000$

$-5.14322000$

$-4.78964700$

$-4.62136400$

5.58683000

6.46496900

4.09202100

1.97929200

2.26067100

0.34941900

$-1.32240800$

$-1.80959200$

$-0.06732000$

$-0.67120100$

$-1.62749800$

$-2.92411900$

$-2.97719000$

$-1.46967800$

$-2.79714300$

$-2.95957500$

$-5.17362500$

$-6.35199100$

$-6.23160300$

$-4.96962200$

$-5.41972600$

$-3.76220300$

$-5.19142100$

$-4.73037400$

$-3.56882900$

0.42948000

$-4.86661800$

$-4.51527100$
Y

0.06271400

1.04578600

1.36878900

0.68203700

$-0.34093400$

$-0.65501500$

$-1.61299800$

$-2.25811300$

$-2.04642600$

$-1.07492100$

$-0.79269200$

$-1.51460100$

$-0.66453500$

$-2.89640500$

0.64547700

0.49445400

0.38256100

1.53767600

$-0.83554200$

2.88346600

$-2.11876200$

$-0.18535200$

1.93829400

2.14955900

0.95395700

$-3.02395800$

$-2.64628200$

$-1.63539900$

$-3.44762700$

$-3.47397900$

$-2.79616500$

$-0.45464800$

$-1.27583500$

1.26788000

1.19619600

$-0.37186800$

1.35814600

1.55518500

1.44794200

$-0.80582800$

$-0.85644300$

3.65635400

3.05880100

$-2.97095500$

$-2.07769700$

$-2.30729800$

$-0.17473200$

2.92580800

3.77360600
Z

$-0.75411200$

0.18353200

1.09735800

1.03315900

0.07989200

$-0.83181700$

$-1.79864800$

$-1.85683000$

$-1.02058900$

$-0.02696300$

0.79431500

0.81697700

1. 56058900

1.45635600

0.85653100

$-0.46828000$

$-0.39223500$

0.20287800

0.21731200

$-0.29904000$

$-0.41280900$

$-1.45876000$

0.25751900

1.83049300

1.73979500

$-2.62533300$

$-1.16066500$

$-0.22503700$

1. 33151200

1.00120800

2.52603200

2.55804300

1. 72179900

1.54332300

0.65633000

$-1.02396800$

$-1.10306000$

1.30427500

$-0.00208500$

0.09147400

1.30911400

0.15986100

0.04595200

$-0.03976400$

$-1.49874200$

$-0.18415600$

1.57189900

$-1.71042500$

$-1.99284300$ 


\section{Atom}

C

C

C

$\mathrm{C}$

C

C

$\mathrm{N}$

C

C

C

$\mathrm{N}$

C

C

C

C

C

$\mathrm{N}$

C

C

C

C

$\mathrm{H}$

Cl

$\mathrm{H}$

$\mathrm{H}$

$\mathrm{H}$

$\mathrm{H}$

$\mathrm{H}$

$\mathrm{H}$

$\mathrm{H}$

$\mathrm{H}$

$\mathrm{H}$

$\mathrm{H}$

$\mathrm{H}$

$\mathrm{H}$

$\mathrm{H}$

$\mathrm{H}$

$\mathrm{H}$

$\mathrm{H}$

$\mathrm{H}$

$\mathrm{H}$

$\mathrm{H}$

$\mathrm{H}$

$\mathrm{H}$

$\mathrm{H}$

$\mathrm{H}$

$\mathrm{H}$

O

$\mathrm{H}$

\section{$\mathrm{x}$}

$-4.82727400$

$-4.88738400$

$-3.82953300$

$-2.70718800$

$-2.59318900$

$-3.67641800$

$-3.69381500$

$-2.61597400$

$-1.48237100$

$-1.42966600$

$-0.38691300$

0.91140100

1. 69853000

0.82157000

2. 03796900

3. 04975600

4. 47329600

4.85681800

4. 96440500

5.11677100

4.81009400

$-5.63682700$

$-6.30321300$

$-3.90595400$

$-1.91357400$

$-2.62056000$

$-0.66488200$

1.45932500

1.83066300

0.24809800

0.35957000

1. 12205800

2.60303900

2. 41020400

1.10333700

2.88948900

2.84163200

4. 11040200

5.78514200

6.03144900

4. 50078500

5.15637700

4. 28692200

5.29981700

5.27672300

3.76532600

$-0.41251600$

6.34094700

6.44310500
Y

0.26321200

1. 27865100

1. 53135700

0.74547500

$-0.31593000$

$-0.55978400$

$-1.55369000$

$-2.30915900$

$-2.17205800$

$-1.17029700$

$-0.98339400$

$-1.66056600$

$-1.40678200$

$-3.14505900$

0.06966300

0.69724800

0.56238200

1. 34222200

$-0.81169300$

2. 80495900

$-1.57077900$

0.06046600

2. 30445500

2. 33305600

0.95791300

$-3.10241700$

$-2.86143500$

$-1.18732200$

$-3.53279300$

$-3.28197600$

$-3.73904000$

$-1.77106300$

$-2.01910700$

0.19372600

0.63836300

0.30303600

1. 76850100

1.28599300

0.92973700

$-0.75422400$

$-1.38301900$

3. 36875400

3. 21722800

$-2.54491000$

$-1.01591200$

$-1.75249300$

$-0.15137700$

2. 90118300

3. 81395500
Z

$-0.65090500$

0.26200700

1.15527400

1.09268200

0.16400800

$-0.72169400$

$-1.65444800$

$-1.70182200$

$-0.89538300$

0.06979600

0.91730600

0.90004500

$-0.39302900$

1.25241700

$-0.62137500$

0.36114500

0.04443400

$-1.13040000$

$-0.02161200$

$-0.81333500$

1.29204900

$-1.33936200$

0.32627000

1.87777700

1.79865300

$-2.44802800$

$-1.04016300$

1.72099000

1.41096800

2.16969200

0.46303700

$-1.24739600$

$-0.35740100$

$-1.64246200$

$-0.56906100$

1.37019400

0.43174600

$-1.94034900$

$-1.53702400$

$-0.25729600$

$-0.84511400$

$-1.75711000$

$-0.22286100$

1.20820700

2.11026100

1. 56127500

1. 48012800

$-0.10885600$

0.17470800 


\section{Atom}

C

C

C

C

C

C

$\mathrm{N}$

C

C

C

$\mathrm{N}$

C

C

C

C

C

$\mathrm{N}$

C

C

C

C

$\mathrm{H}$

$\mathrm{Cl}$

$\mathrm{H}$

$\mathrm{H}$

$\mathrm{H}$

$\mathrm{H}$

$\mathrm{H}$

$\mathrm{H}$

$\mathrm{H}$

$\mathrm{H}$

$\mathrm{H}$

$\mathrm{H}$

$\mathrm{H}$

$\mathrm{H}$

$\mathrm{H}$

$\mathrm{H}$

$\mathrm{H}$

$\mathrm{H}$

$\mathrm{H}$

$\mathrm{H}$

$\mathrm{H}$

$\mathrm{H}$

$\mathrm{H}$

$\mathrm{H}$

$\mathrm{H}$

$\mathrm{H}$

O

$\mathrm{H}$

\section{$\mathrm{x}$}

$-4.88161100$

$-5.27175400$

$-4.58532400$

$-3.47045400$

$-2.99974800$

$-3.74070600$

$-3.42421600$

$-2.38252800$

$-1.55448700$

$-1.83024100$

$-1.03665400$

0.09306100

1. 21730300

$-0.36283700$

2.47263800

3. 69686200

4. 90575800

4. 93712100

6.12264600

6.18916300

6.48074000

$-5.42163400$

$-6.67777800$

$-4.95041000$

$-2.94304500$

$-2.14848300$

$-0.68709000$

0.49163100

0.48785500

$-1.12401900$

$-0.77328300$

1. 47573900

0.84482100

2.67859600

2.30639000

3. 86710200

3. 47953200

4.83628900

4.07488200

6.94712300

6.00016200

6.07204700

7.06494500

7.39069700

6.65316800

5.68185600

$-0.63018400$

6.35401200

7. 12215900
$Y$

$-0.12673900$

0.89995000

1.20877700

0.48765100

$-0.55524300$

$-0.89610000$

$-1.94922500$

$-2.67220900$

$-2.39826600$

$-1.31957400$

$-0.98256900$

$-0.04394400$

$-0.69704200$

1. 27164700

0.16781300

$-0.64683400$

0.16260500

0.88851900

$-0.59708300$

1. 71364300

$-1.68818600$

$-0.38286900$

1.85524200

1.99855900

0.68686500

$-3.52098200$

$-3.01826200$

0.16542900

1. 94886600

1. 75499300

1.11755000

$-1.64922100$

$-0.94206800$

0.63882000

0.97826400

$-1.41746000$

$-1.18284600$

0.20537100

1. 56180500

0.11722200

$-1.04970500$

2. 22302600

1.05605000

$-2.20633500$

$-1.27166000$

$-2.42950000$

$-1.83893000$

2.64259400

3.18080400
Z

1. 10235800

0.28811000

$-0.90608300$

$-1.23894600$

$-0.40484400$

0.75698400

1.56319300

1.21492100

0.10697400

$-0.70233000$

$-1.82312600$

$-1.59280300$

$-0.77587000$

$-0.97976500$

$-0.67404200$

$-0.26625700$

$-0.16119100$

1.10369900

$-0.43767600$

1.33428500

0.57527000

2.00431500

0.70654200

$-1.54854900$

$-2.16272000$

1.85330300

$-0.08647300$

$-2.59106600$

$-0.89621300$

$-1.59215600$

0.02158900

$-1.25634700$

0.22564900

$-1.64185700$

0.04305100

$-1.02741100$

0.67680000

1. 96866500

1.13213600

$-0.52425000$

$-1.42716200$

2. 30004700

1. 42063600

0.26584300

1. 57131400

0.65522300

$-2.18759700$

0.27843400

0.48849700 


\section{HCQ-M15-Structure}

WB97x-D/6-31G(d,p) - Water fully optimized XYZ coordinates

\section{Atom}

C

C

C

C

C

C

$\mathrm{N}$

C

C

C

$\mathrm{N}$

C

C

C

C

C

$\mathrm{N}$

C

C

C

C

$\mathrm{H}$

Cl

$\mathrm{H}$

$\mathrm{H}$

$\mathrm{H}$

$\mathrm{H}$

$\mathrm{H}$

$\mathrm{H}$

$\mathrm{H}$

$\mathrm{H}$

$\mathrm{H}$

$\mathrm{H}$

$\mathrm{H}$

$\mathrm{H}$

$\mathrm{H}$

$\mathrm{H}$

$\mathrm{H}$

$\mathrm{H}$

$\mathrm{H}$

$\mathrm{H}$

$\mathrm{H}$

$\mathrm{H}$

$\mathrm{H}$

$\mathrm{H}$

$\mathrm{H}$

$\mathrm{H}$

O

$\mathrm{H}$ $\mathrm{x}$

2.74411800

3. 21658200

2.49990000

1. 28506800

0.72689700

1. 49879300

1. 13477500

$-0.00790700$

$-0.86584200$

$-0.54727300$

$-1.42636300$

$-1.74024000$

$-1.79788700$

$-3.06004400$

$-2.81021300$

$-2.43162200$

$-1.11639800$

$-1.10077700$

$-0.38872700$

$-1.55699700$

1.07099400

3. 29972100

4. 75591700

2.91453500

0.76021400

$-0.29649200$

$-1.80366000$

$-0.97560100$

$-3.30115300$

$-3.00092100$

$-3.87263600$

$-2.00975000$

$-0.81789500$

$-3.81964500$

$-2.84980100$

$-3.21068500$

$-2.36624900$

$-0.08248200$

$-1.71183400$

$-0.86256400$

$-0.42741200$

$-1.35066700$

$-0.96864900$

1. 62277200

1.17939400

1. 52833700

$-2.24565700$

$-2.94308900$

$-3.19013100$
$\mathbf{Y}$

0.08623200

$-0.38336500$

$-1.33168000$

$-1.76719500$

$-1.28610400$

$-0.37504400$

0.08706100

$-0.37042800$

$-1.23786400$

$-1.69661000$

$-2.54167100$

$-2.64506800$

$-1.28795300$

$-3.39977800$

$-0.29916700$

1.13688200

1.49423700

1.81030500

2.43825100

3. 20189600

2.57341300

0.79890600

0.18339000

$-1.71582000$

$-2.51375400$

$-0.01294600$

$-1.52662300$

$-3.25861900$

$-3.56959400$

$-4.36666800$

$-2.82084400$

$-1.47138600$

$-0.80688600$

$-0.51038800$

$-0.40128300$

1.83957100

1.19562200

1.67741100

1.06887400

3.43727800

2.05616600

3. 31530100

3. 96686100

3. 14853400

3.08732600

1.58352300

$-2.70735000$

3. 35716900

4. 25407200
Z

0.91682000

$-0.27504900$

$-1.03131600$

$-0.57150900$

0.64024800

1.41807600

2.64566000

3.12041100

2.44284700

1.16745200

0.54042800

$-0.89404700$

$-1.60181400$

$-1.00465400$

$-1.02561800$

$-1.39612300$

$-0.86647800$

0.55491100

$-1.69989700$

0.97636800

$-1.28344900$

1.51219600

$-0.88428100$

$-1.95380400$

$-1.14996800$

4.10554000

2.90282500

$-1.38061500$

$-2.05501100$

$-0.49976700$

$-0.55572200$

$-2.66086200$

$-1.55088700$

$-1.39106100$

0.06501600

$-1.07765900$

$-2.48790500$

0.93477400

1.07605400

$-1.72465300$

$-2.72455100$

2.05087300

0.45072800

$-2.02931000$

$-0.32388300$

$-1.18824500$

1.10795300

0.70862800

0.95247300 
Atom

$\mathrm{C}$

C

C

C

C

C

$\mathrm{N}$

C

C

C

$\mathrm{N}$

C

C

C

C

C

$\mathrm{N}$

C

C

C

C

$\mathrm{H}$

$\mathrm{Cl}$

$\mathrm{H}$

$\mathrm{H}$

$\mathrm{H}$

$\mathrm{H}$

$\mathrm{H}$

$\mathrm{H}$

$\mathrm{H}$

$\mathrm{H}$

$\mathrm{H}$

$\mathrm{H}$

$\mathrm{H}$

$\mathrm{H}$

$\mathrm{H}$

$\mathrm{H}$

$\mathrm{H}$

$\mathrm{H}$

$\mathrm{H}$

$\mathrm{H}$

$\mathrm{H}$

$\mathrm{H}$

$\mathrm{H}$

$\mathrm{H}$

$\mathrm{H}$

$\mathrm{H}$

O

$\mathrm{H}$

\section{$\mathrm{x}$}

4.49094800

4. 54121900

3.57835500

2. 56190200

2. 46318500

3.44439800

3. 45284100

2.46523000

1. 43482200

1.40608000

0.45638100

$-0.66199900$

$-1.76046900$

$-1.18275900$

$-2.44148800$

$-3.44448600$

$-4.20136400$

$-3.40263600$

$-5.44254400$

$-3.83863100$

$-6.53284800$

5.23050900

5.83330200

3. 64344000

1.82813100

2.46515300

0.67530500

$-0.27240300$

$-2.04389500$

$-0.41178000$

$-1.50030400$

$-2.50561100$

$-1.32344800$

$-2.97468500$

$-1.67710500$

$-4.15306500$

$-2.92718800$

$-3.42985400$

$-2.35782200$

$-5.25152100$

$-5.80415200$

$-3.30051800$

$-4.91209500$

$-7.44441400$

$-6.23695700$

$-6.75810600$

0.47886700

$-3.54750900$

$-3.84719600$
$Y$

0.31344700

1. 30267900

1. 40170500

0.48155600

$-0.55924000$

$-0.64228200$

$-1.59582600$

$-2.46771800$

$-2.49316500$

$-1.54227400$

$-1.53111600$

$-2.46278900$

$-2.14466500$

$-2.46822400$

$-0.79499400$

$-0.51192800$

0.71978400

1.89903500

0.71497500

3.13275400

$-0.13975500$

0.23421000

2.48327200

2.19341600

0.57865200

$-3.22944500$

$-3.25924900$

$-3.46207700$

$-3.13276300$

$-2.80959900$

$-1.46710500$

$-2.94710600$

$-2.17879100$

$-0.76995900$

$-0.01062100$

$-1.34484700$

$-0.50563400$

2.11813300

1.70620200

0.39275800

1. 74466500

4. 00231000

3. 31520100

$-0.09514700$

$-1.18843100$

0.22299800

$-0.80045100$

2. 93698300

3.71659600
Z

$-0.88348400$

0.05917700

1. 08111300

1.12241400

0.16971700

$-0.85370300$

$-1.82938800$

$-1.78664700$

$-0.84060700$

0.17602500

1.13988200

1. 22287700

0.19703000

2.65496100

0.40066700

$-0.71321700$

$-0.50989900$

$-0.83155900$

$-1.28369700$

$-0.06363200$

$-0.65365700$

$-1.66992400$

0.01054300

1. 81594400

1.91381900

$-2.56394300$

$-0.91535300$

1.00138100

2.73614600

3.34736500

2. 96034500

0.24028500

$-0.80713100$

1.35604300

0.44391600

$-0.74822700$

$-1.69086900$

$-1.91341000$

$-0.57616600$

$-2.32319900$

$-1.34570200$

$-0.46386000$

$-0.21747800$

$-1.25296000$

$-0.57647400$

0.35116100

1. 83127900

1.30737600

1. 78194100 
Atom

C

$\mathrm{C}$

C

$\mathrm{C}$

C

C

$\mathrm{N}$

C

C

C

$\mathrm{N}$

C

$\mathrm{C}$

C

C

C

$\mathrm{N}$

C

$\mathrm{C}$

C

C

$\mathrm{H}$

$\mathrm{Cl}$

$\mathrm{H}$

$\mathrm{H}$

$\mathrm{H}$

$\mathrm{H}$

$\mathrm{H}$

$\mathrm{H}$

$\mathrm{H}$

$\mathrm{H}$

$\mathrm{H}$

$\mathrm{H}$

$\mathrm{H}$

$\mathrm{H}$

$\mathrm{H}$

$\mathrm{H}$

$\mathrm{H}$

$\mathrm{H}$

$\mathrm{H}$

$\mathrm{H}$

$\mathrm{H}$

$\mathrm{H}$

$\mathrm{H}$

$\mathrm{H}$

$\mathrm{H}$

$\mathrm{H}$

$\mathrm{O}$

$\mathrm{H}$

\section{$\mathrm{x}$}

3.62896800

3.53286300

2.35542500

1.27437400

1.31444000

2.51953400

2.68776000

1.64149800

0.39124900

0.18333700

$-0.99715300$

$-2.19429000$

$-3.44159000$

$-2.12139200$

$-3.85715200$

$-3.32019700$

$-1.87956200$

$-1.36002200$

$-1.41100200$

$-1.95784000$

$-2.12514400$

4.53967300

4.91327600

2.31650700

0.38013600

1.77254400

$-0.39632700$

$-2.28232400$

$-2.95195100$

$-2.18332200$

$-1.18761900$

$-4.26216300$

$-3.31228400$

$-4.94532000$

$-3.61549800$

$-3.89392200$

$-3.52684000$

$-1.51705400$

$-0.27742800$

$-0.34079200$

$-1.49075500$

$-3.04387600$

$-1.73721300$

$-1.69738500$

$-2.02022200$

$-3.19194600$

$-1.19197900$

$-1.48982300$

$-0.54541700$
Y

$-0.48503000$

0.69560800

1.07114700

0.22662700

$-0.99017100$

$-1.36662200$

$-2.54386000$

$-3.34508500$

$-3.07944000$

$-1.88141300$

$-1.52449000$

$-2.34845500$

$-1.51600500$

$-3.54321800$

$-0.45596900$

0.95636800

1.10487000

2.35466000

0.76085200

3.67279300

1.41175200

$-0.78070000$

1.77154000

2.00016300

0.49665800

$-4.28830000$

$-3.81131100$

$-2.71549000$

$-4.22740300$

$-3.19181600$

$-4.09585700$

$-2.22969900$

$-1.04706200$

$-0.36538600$

$-0.79592200$

1.64268800

1.24751900

2.33766900

2.37330400

0.98829500

$-0.32706400$

3.66200300

3.82679000

1.03318700

2.49764400

1.17320500

$-0.51599600$

4.75852400

4.84247600
Z

0.38765100

$-0.29704900$

$-0.97195000$

$-0.92947600$

$-0.21081900$

0.43619000

1. 10489900

1. 12586200

0.55095100

$-0.12449500$

$-0.70033800$

$-0.57946900$

$-0.88777600$

$-1.53178100$

0.14353300

$-0.08266100$

0.11959000

$-0.42755400$

1. 46680800

0.08211600

2. 65065500

0.89174400

$-0.36052000$

$-1.52550500$

$-1.47751500$

1.65145600

0.65897700

0.45538700

$-1.34652400$

$-2.56477500$

$-1.41601200$

$-0.99526600$

$-1.87140300$

0.11451700

1. 15709100

0.55741400

$-1.11805100$

$-1.51238000$

$-0.25995400$

1. 49487200

1. 57560200

$-0.03045600$

1. 14470600

3.58111700

2.64993400

2.65434400

$-0.70268900$

$-0.69664100$

$-0.53814400$ 
Atom

$\mathrm{C}$

$\mathrm{C}$

C

C

C

C

$\mathrm{N}$

C

C

C

$\mathrm{N}$

C

C

C

C

C

$\mathrm{N}$

C

C

C

C

$\mathrm{H}$

$\mathrm{Cl}$

$\mathrm{H}$

$\mathrm{H}$

$\mathrm{H}$

$\mathrm{H}$

$\mathrm{H}$

$\mathrm{H}$

$\mathrm{H}$

$\mathrm{H}$

$\mathrm{H}$

$\mathrm{H}$

$\mathrm{H}$

$\mathrm{H}$

$\mathrm{H}$

$\mathrm{H}$

$\mathrm{H}$

$\mathrm{H}$

$\mathrm{H}$

$\mathrm{H}$

$\mathrm{H}$

$\mathrm{H}$

$\mathrm{H}$

$\mathrm{H}$

$\mathrm{H}$

$\mathrm{H}$

$\mathrm{O}$

$\mathrm{H}$

\section{$\mathrm{x}$}

5.04307500

4.96318600

3.76248700

2.64879000

2.68030600

3.89863300

4.04751200

2.97394500

1.72380000

1.53499100

0.33935700

$-0.82175600$

$-2.04854900$

$-0.61744600$

$-3.36603200$

$-4.55114300$

$-4.70860800$

$-5.14463400$

$-5.55191200$

$-3.95021300$

$-5.36430900$

5.96557200

6.38512000

3.72782700

1.73482600

3.08986500

0.92091600

$-0.95279400$

$-1.43639700$

$-0.57710200$

0.31668600

$-1.91737400$

$-2.11578200$

$-3.38046900$

$-3.47124300$

$-5.47656900$

$-4.38386500$

$-5.62055000$

$-5.89689600$

$-5.28174500$

$-6.61565200$

$-3.28906400$

$-3.37182500$

$-5.98186500$

$-4.31913900$

$-5.64737400$

0.31996700

$-4.33298700$

$-4.85024900$
$\mathrm{Y}$

$-0.00306800$

1.28383000

1.81942700

1.01969700

$-0.32107800$

$-0.84002000$

$-2.10972800$

$-2.87283600$

$-2.49374700$

$-1.19909100$

$-0.75069900$

$-1.59788100$

$-0.69413400$

$-2.46181700$

$-1.44352800$

$-0.48185400$

0.18846800

1.56888100

$-0.58822300$

2.47661300

$-0.20693300$

$-0.41466100$

2.30454900

2.84204500

1.45304000

$-3.88482200$

$-3.21770400$

$-2.24661100$

$-3.17318800$

$-1.82526900$

$-3.02367900$

$-0.01112400$

$-0.07337100$

$-1.99739100$

$-2.18341600$

$-1.00839100$

0.27652300

1.90818700

1.67795100

$-1.64118400$

$-0.50499800$

2.46016400

2.08954900

$-0.84236500$

$-0.33073800$

0.83048000

0.14179600

3.82331300

3.85363200
Z

0.54374800

0.08947900

$-0.41260300$

$-0.45592700$

$-0.00490800$

0.50902000

0.98365200

0.93142300

0.42764300

$-0.04685600$

$-0.50089700$

$-0.75053400$

$-0.85351300$

$-1.99500900$

$-1.01786100$

$-1.00127400$

0.28911100

0.13733000

1.19373000

$-0.14184600$

2.65446000

0.93169200

0.12343900

$-0.76320400$

$-0.84382400$

1. 31112500

0.41956100

0.12281100

$-2.11642900$

$-2.88260700$

$-1.92893300$

$-1.70553700$

0.04483700

$-1.96141300$

$-0.21508700$

$-1.28310900$

$-1.77330700$

1.05958800

$-0.66467000$

1.07039100

0.91215900

0.72688000

$-0.99245700$

3.29276000

2. 94233200

2.84846200

$-0.96748400$

$-0.34613200$

$-1.15601700$ 
Atom

C

C

C

$\mathrm{C}$

$\mathrm{C}$

C

$\mathrm{N}$

C

C

C

$\mathrm{N}$

C

C

C

C

C

N

C

C

C

C

$\mathrm{H}$

$\mathrm{Cl}$

$\mathrm{H}$

H

H

$\mathrm{H}$

H

H

$\mathrm{H}$

$\mathrm{H}$

$\mathrm{H}$

$\mathrm{H}$

$\mathrm{H}$

$\mathrm{H}$

$\mathrm{H}$

$\mathrm{H}$

$\mathrm{H}$

$\mathrm{H}$

$\mathrm{H}$

$\mathrm{H}$

$\mathrm{H}$

$\mathrm{H}$

$\mathrm{H}$

$\mathrm{H}$

$\mathrm{H}$

$\mathrm{H}$

$\mathrm{O}$

$\mathrm{H}$ $\mathrm{x}$

$-2.66067500$

$-2.64533200$

$-2.23468400$

$-1.81954500$

$-1.80587300$

$-2.24629200$

$-2.27423000$

$-1.87771000$

$-1.39183100$

$-1.29903300$

$-0.68341700$

0.80078100

1.57069500

1.21216400

1.59406300

2.69228300

2.37829700

3.53806200

1.16995200

4.17498200

0.94154600

$-2.98196100$

$-3.14028900$

$-2.23836300$

$-1.47435700$

$-1.92761300$

$-1.04637400$

1.07406000

2.29082900

0.98972300

0.70684800

1.20538100

2.60909000

1.74700700

0.62621700

2.92842700

3.60580100

4.30517400

3.26757300

1.19792000

0.29701700

5.20309100

4.22741500

$-0.00972500$

1.74401600

0.87290300

$-1.09433300$

3.40824500

3.76732400
$\mathrm{Y}$

1.09604700

1.71980900

1.05607000

$-0.24637100$

$-0.93706300$

$-0.25715300$

$-0.82785000$

$-2.07942500$

$-2.83682800$

$-2.26785100$

$-2.97694800$

$-2.90470900$

$-2.91913600$

$-1.76564900$

$-1.69543200$

$-0.64687500$

0.38416300

1.14076800

1.16512300

2.12086600

1.65227800

1.60297800

3.39233600

1.58218800

$-0.73828700$

$-2.53487800$

$-3.85002800$

$-3.84313500$

$-1.79858700$

$-0.79077500$

$-1.86128000$

$-3.77997800$

$-3.15480500$

$-2.09777300$

$-1.19533500$

$-0.21112700$

$-1.15025500$

0.41837900

1.71237300

2.02878800

0.57567200

2.32936600

1.66859200

2.19179800

2.32306900

0.81788600

$-2.72972300$

3.31403200

3.88055100
Z

$-0.78658300$

0.42890100

1. 60382400

1.52218700

0.28395800

$-0.88397600$

$-2.12066400$

$-2.20595600$

$-1.12527100$

0.12566000

1.17028600

1. 31745000

$-0.01107300$

2. 24505200

$-0.95654800$

$-0.74307700$

0.23346600

0.66625900

$-0.03076800$

$-0.31796400$

$-1.46242400$

$-1.68697600$

0.54977500

2.54930300

2. 42295100

$-3.19152200$

$-1.28227100$

1.81265900

2.41865400

1.81070400

3. 21141600

$-0.57925100$

0.24894600

$-1.96239500$

$-1.00124600$

$-1.72939300$

$-0.40585100$

0.96586000

1. 56115100

0.63807700

0.26830600

0.00917100

$-1.31848300$

$-1.50571700$

$-1.77749400$

$-2.16591800$

2. 06283000

$-0.33683800$

$-1.02449800$ 
Atom

C

C

$\mathrm{C}$

$\mathrm{C}$

$\mathrm{C}$

C

N

C

$\mathrm{C}$

C

$\mathrm{N}$

C

C

C

C

C

$\mathrm{N}$

C

C

C

C

$\mathrm{H}$

$\mathrm{Cl}$

$\mathrm{H}$

$\mathrm{H}$

$\mathrm{H}$

$\mathrm{H}$

$\mathrm{H}$

$\mathrm{H}$

$\mathrm{H}$

$\mathrm{H}$

$\mathrm{H}$

$\mathrm{H}$

$\mathrm{H}$

$\mathrm{H}$

$\mathrm{H}$

$\mathrm{H}$

$\mathrm{H}$

$\mathrm{H}$

$\mathrm{H}$

$\mathrm{H}$

$\mathrm{H}$

$\mathrm{H}$

$\mathrm{H}$

$\mathrm{H}$

$\mathrm{H}$

$\mathrm{H}$

$\mathrm{O}$

$\mathrm{H}$

\section{$\mathrm{x}$}

3.71832800

4.62271200

4.36182400

3.16670400

2.19654800

2.47926200

1.62825400

0.48800400

0.09595000

0.92913900

0.60610300

$-0.63025900$

$-1.65483200$

$-0.29243500$

$-2.20520100$

$-3.28175200$

$-3.85477600$

$-2.89408600$

$-4.53657900$

$-3.47355000$

$-5.89456400$

3.91338600

6.15100400

5.09692300

2.98971300

$-0.20359100$

$-0.87141300$

$-1.03988700$

$-1.19151700$

0.44775600

0.10945500

$-1.17239100$

$-2.48654600$

$-2.64820600$

$-1.38665500$

$-4.09717100$

$-2.88093300$

$-2.47058500$

$-2.04557900$

$-3.90859700$

$-4.69068800$

$-2.78251300$

$-4.42945200$

$-6.39428100$

$-5.78732600$

$-6.52977000$

1.23155300

$-3.63685600$

$-4.07224000$
$\mathrm{Y}$

$-1.47756300$

$-0.79993900$

0.49814100

1.09183700

0.43347000

$-0.87617200$

$-1.61394900$

$-1.02895000$

0.25521500

1.02596700

2.26879800

2.99773300

2.80979100

4.47376900

1.39239200

1.05668800

$-0.28411900$

$-1.30324800$

$-0.54293200$

$-2.69043000$

$-1.21202900$

$-2.47384200$

$-1.54928100$

1.01304800

2.09372300

$-1.61319800$

0.61889800

2.62636100

5.03531200

4.60706700

4.89086700

3.13011800

3.50283700

1.29291100

0.66725600

1.78236600

1.19542600

$-0.98949000$

$-1.39859600$

$-1.15214200$

0.41648600

$-3.26248600$

$-2.60199500$

$-1.31230500$

$-2.21010100$

$-0.61137900$

2.72035500

$-3.33405800$

$-4.17482300$
Z

0.33831900

$-0.43131900$

$-0.90944300$

$-0.59148000$

0.20040800

0.67382600

1. 44264200

1.75221500

1.36531100

0.56095200

0.13519300

0.40082100

$-0.72797700$

0.58314000

$-0.88677900$

0.15763100

0.08908500

0.48967500

$-1.18520000$

0.69585700

$-1.00855100$

0.71124800

$-0.83979000$

$-1.51336300$

$-0.96382700$

2. 35626200

1.67911300

1. 34351400

0.84114100

1. 37333700

$-0.34563500$

$-1.65750100$

$-0.55340400$

$-1.88271000$

$-0.83796600$

0.05319900

1.16939400

1.44963300

$-0.21150000$

$-1.84857100$

$-1.68775500$

1.32739200

1.22749500

$-1.97629400$

$-0.58201300$

$-0.35328800$

$-0.51209700$

$-0.55792600$

$-0.39884300$ 
Atom

$\mathrm{C}$

C

$\mathrm{C}$

C

C

C

$\mathrm{N}$

C

C

C

$\mathrm{N}$

C

C

C

C

C

$\mathrm{N}$

C

C

C

C

$\mathrm{H}$

$\mathrm{Cl}$

$\mathrm{H}$

$\mathrm{H}$

$\mathrm{H}$

$\mathrm{H}$

$\mathrm{H}$

$\mathrm{H}$

$\mathrm{H}$

$\mathrm{H}$

$\mathrm{H}$

$\mathrm{H}$

$\mathrm{H}$

$\mathrm{H}$

$\mathrm{H}$

$\mathrm{H}$

$\mathrm{H}$

$\mathrm{H}$

$\mathrm{H}$

$\mathrm{H}$

$\mathrm{H}$

$\mathrm{H}$

$\mathrm{H}$

$\mathrm{H}$

$\mathrm{H}$

$\mathrm{H}$

O

$\mathrm{H}$ $\mathrm{x}$

$-2.63461800$

$-1.57757700$

$-0.78372400$

$-1.07140900$

$-2.11220800$

$-2.93209400$

$-4.01383300$

$-4.28108900$

$-3.50496700$

$-2.39685000$

$-1.58433800$

$-0.42937900$

0.60231400

0.15439900

1. 86327800

2.67225900

2.70647500

3. 38414900

3. 12398500

2. 78710600

4.53968600

$-3.25667100$

$-1.20477700$

0.04117200

$-0.50612300$

$-5.15323000$

$-3.77151200$

$-0.78497200$

0.94394500

$-0.61359500$

0.57705300

0.15310900

0.89253400

1. 60767600

2.47583600

3.67784300

2.19163600

3.27716100

4. 46589400

2. 40520400

3. 02642200

3. 21662400

1.70270600

4.75400200

4.65605800

5.29616800

$-2.16927900$

3. 06852300

2. 64964500
$Y$

2.09471900

2.58097600

1. 74927700

0.41162500

$-0.15314900$

0.70767700

0.27373500

$-1.01234500$

$-1.96141800$

$-1.55336000$

$-2.46782000$

$-3.04338200$

$-1.97644100$

$-4.14874100$

$-2.49953900$

$-1.36256000$

$-0.15515400$

$-0.31480300$

1. 02289000

0.53120300

0.97762900

2.73957700

4.29007700

2.16775200

$-0.24011800$

$-1.34988100$

$-3.01049000$

$-3.48780300$

$-4.68010200$

$-4.87609000$

$-3.72659000$

$-1.20962600$

$-1.47302300$

$-3.22315100$

$-3.04647300$

$-1.73605300$

$-1.07850100$

$-1.35504400$

$-0.12179400$

1.15115900

1.88540600

0.19810800

0.35664600

1. 90850100

0.15240200

0.86105900

$-3.25349700$

1. 90656800

2. 40689700
Z

0.51388100

$-0.20396900$

$-1.02311600$

$-1.08083600$

$-0.30467900$

0.47181200

1. 17923400

1. 12234400

0.42822900

$-0.27852400$

$-0.98781400$

$-0.24741000$

0.09150400

$-1.11520600$

0.77128000

1. 40764900

0.58977800

$-0.68902300$

1. 35013400

$-1.80436400$

1.93739400

1. 12049500

$-0.14101600$

$-1.58839100$

$-1.73291400$

1.67510200

0.47185000

0.69630200

$-0.58174500$

$-1.39059300$

$-2.03043000$

0.72965900

$-0.83399500$

1.55330800

0.04606500

1. 66647200

2. 35092800

$-1.00839400$

$-0.62945300$

2.16728700

0.68977800

$-2.76107000$

$-1.83310300$

2.46936200

2.64416700

1.15662800

$-1.25557600$

$-1.58215500$

$-2.28822000$ 
Atom

$\mathrm{C}$

C

C

C

C

C

$\mathrm{N}$

C

C

C

$\mathrm{N}$

C

C

C

C

C

$\mathrm{N}$

C

C

C

C

$\mathrm{H}$

$\mathrm{Cl}$

$\mathrm{H}$

$\mathrm{H}$

$\mathrm{H}$

$\mathrm{H}$

$\mathrm{H}$

$\mathrm{H}$

$\mathrm{H}$

$\mathrm{H}$

$\mathrm{H}$

$\mathrm{H}$

$\mathrm{H}$

$\mathrm{H}$

$\mathrm{H}$

$\mathrm{H}$

$\mathrm{H}$

$\mathrm{H}$

$\mathrm{H}$

$\mathrm{H}$

$\mathrm{H}$

$\mathrm{H}$

$\mathrm{H}$

$\mathrm{H}$

$\mathrm{H}$

$\mathrm{H}$

$\mathrm{O}$

$\mathrm{H}$

\section{$\mathrm{x}$}

3.83296900

3. 65148100

2.43656400

1. 40522300

1. 53150900

2. 77389600

3. 01900800

2.01193000

0.72959800

0.44440400

$-0.76087800$

$-1.93071500$

$-2.70073100$

$-2.80490300$

$-3.47859800$

$-2.67663900$

$-1.81348900$

$-2.53225600$

$-0.90329700$

$-3.57111700$

0.02932900

4. 77306300

4. 97310900

2. 33218300

0.47359500

2. 20362800

$-0.02684500$

$-1.57524100$

$-3.73651600$

$-2.28285700$

$-3.06091100$

$-3.41589200$

$-1.99247500$

$-4.06393500$

$-4.21656800$

$-2.02987100$

$-3.37524100$

$-1.77248600$

$-3.03724900$

$-0.30142200$

$-1.46251400$

$-3.76068300$

$-3.18659300$

0.83691900

0.47343200

$-0.47906000$

$-1.00719600$

$-4.75683200$

$-5.40182800$
$Y$

0.21183900

$-1.02003500$

$-1.38902800$

$-0.48487800$

0.78724900

1. 15420400

2.37441600

3. 22517300

2. 97252700

1. 73469300

1. 36998200

2. 23954000

2.10570300

1. 98820100

0.80153800

$-0.39856100$

$-1.05819600$

$-1.68300500$

$-2.02138300$

$-2.75027900$

$-1.40913700$

0.49884600

$-2.16938100$

$-2.36152300$

$-0.76084100$

4. 19890100

3.73981200

3.27029900

2. 55391300

2.28848400

0.93095100

2. 93313200

2.26700500

0.52558800

1. 00927500

$-0.04199500$

$-1.12635600$

$-2.12435600$

$-0.90796000$

$-2.45802300$

$-2.84749400$

$-3.32508600$

$-3.45113800$

$-2.11148100$

$-0.48392300$

$-1.19351600$

0.38989400

$-2.11527800$

$-2.80030000$
Z

0.23013400

$-0.33617300$

$-0.94643400$

$-0.95818700$

$-0.35200000$

0.22583800

0.78468900

0.76995000

0.26423100

$-0.30007400$

$-0.81949100$

$-0.76260800$

0.56071100

$-1.98766400$

0.80034100

1.30671200

0.31755500

$-0.79710600$

0.94975800

$-0.47243900$

1. 98689300

0.68272400

$-0.34227800$

$-1.40978500$

$-1.43930900$

1. 21438900

0.35655000

$-0.83003900$

$-1.91512100$

$-2.89688900$

$-2.08444200$

0.59595600

1. 38275800

$-0.08192300$

1. 58033000

2.11242300

1.74145500

$-1.44989000$

$-1.38101100$

0.14680100

1. 41494600

$-1.38836900$

0.28081900

2. 20181700

1.61277100

2.92786100

$-0.61061700$

$-0.02323800$

0.16986000 
Atom

C

C

C

C

C

C

$\mathrm{N}$

C

C

C

$\mathrm{N}$

C

C

C

C

C

$\mathrm{N}$

C

C

C

C

$\mathrm{H}$

$\mathrm{Cl}$

$\mathrm{H}$

$\mathrm{H}$

$\mathrm{H}$

$\mathrm{H}$

$\mathrm{H}$

$\mathrm{H}$

$\mathrm{H}$

$\mathrm{H}$

$\mathrm{H}$

$\mathrm{H}$

$\mathrm{H}$

$\mathrm{H}$

$\mathrm{H}$

$\mathrm{H}$

$\mathrm{H}$

$\mathrm{H}$

$\mathrm{H}$

$\mathrm{H}$

$\mathrm{H}$

$\mathrm{H}$

$\mathrm{H}$

$\mathrm{H}$

$\mathrm{H}$

$\mathrm{H}$

$\mathrm{O}$

$\mathrm{H}$

\section{$\mathrm{x}$}

$-4.92295100$

$-5.34560800$

$-4.43742300$

$-3.09445600$

$-2.60701600$

$-3.53802300$

$-3.18700800$

$-1.89262200$

$-0.87466700$

$-1.20226000$

$-0.26809600$

1. 17098900

1.68903300

1.85976000

2.77828600

4. 20815700

4. 65950200

5.43865200

5.39794600

4. 71497800

4.51090800

$-5.62040400$

$-7.05861700$

$-4.80036900$

$-2.41140600$

$-1.61016900$

0.15548000

1. 36250900

2. 92888200

1. 46444500

1. 70712400

0.83390900

2.04690900

2.69907100

2.59069300

4. 25417400

4. 90085400

6.36997100

5. 73123700

5.86986900

6.21786900

5. 40989100

4.45918100

5. 10901600

3.73162300

4.02163300

$-0.55741300$

3. 55994000

3. 09951000
$Y$

0.72400400
-0.52545100
-1.53912400
-1.25910100
0.01706800
1.02752500
2.29287900
2.54802700
1.64589700
0.35016300

$-0.55271900$

$-0.30975000$

$-0.23169200$

$-1.38297300$

0.82489700

0.28127900

$-0.38036100$

0.49668400

$-1.61079700$

1.74702700

$-2.74649700$

1. 50338200

$-0.88843700$

$-2.51866300$

$-2.05259600$

3. 55525300

1.97064500

0.65219900

$-1.16791500$

$-1.40136200$

$-2.36931900$

0.00142700

$-1.22165600$

1. 60957300

1. 30848200

$-0.44086500$

1. 09351000

0.82657000

$-0.07354000$

$-1.92263000$

$-1.43308400$

2.32366900

2.37507600

$-3.64675700$

$-2.96510800$

$-2.50425600$

$-1.49460900$

1. 38092800

2. 18686700
Z

$-0.25984900$

0.10138300

0.46135400

0.44810200

0.08124400

$-0.27879700$

$-0.64947400$

$-0.66557500$

$-0.33834500$

0.05857900

0.42524100

0.38355700

$-1.07321000$

1.21030900

$-1.32332900$

$-1.37908000$

$-0.15661600$

0.70552900

$-0.42796900$

1.16432800

$-0.92145400$

$-0.53749300$

0.12278600

0.74342700

0.72793400

$-0.96327500$

$-0.39557000$

0.87279600

1. 22502500

2. 22854600

0.75687900

$-1.71271200$

$-1.37991600$

$-0.56434800$

$-2.28756900$

$-2.19944500$

$-1.65610100$

0.20807800

1. 59226400

0.50819600

$-1.14690300$

1.78769400

0.30082900

$-1.07840800$

$-0.18944700$

$-1.86835800$

0.62921700

1.89434000

2. 14185100 
Atom

$\mathrm{C}$

C

C

C

C

C

$\mathrm{N}$

C

C

C

$\mathrm{N}$

C

C

C

C

C

$\mathrm{N}$

C

C

C

C

$\mathrm{H}$

$\mathrm{Cl}$

$\mathrm{H}$

$\mathrm{H}$

$\mathrm{H}$

$\mathrm{H}$

$\mathrm{H}$

$\mathrm{H}$

$\mathrm{H}$

$\mathrm{H}$

$\mathrm{H}$

$\mathrm{H}$

$\mathrm{H}$

$\mathrm{H}$

$\mathrm{H}$

$\mathrm{H}$

$\mathrm{H}$

$\mathrm{H}$

$\mathrm{H}$

$\mathrm{H}$

$\mathrm{H}$

$\mathrm{H}$

$\mathrm{H}$

$\mathrm{H}$

$\mathrm{H}$

$\mathrm{H}$

$\mathrm{O}$

$\mathrm{H}$

\section{$\mathrm{x}$}

3.94686700

3. 66776500

2.36405100

1.35294300

1.58670200

2.90814400

3. 25030400

2. 25804800

0.91704200

0.53178500

$-0.73576100$

$-1.86111800$

$-3.03627500$

$-2.26577600$

$-3.80744600$

$-3.30382200$

$-1.99632000$

$-1.94725000$

$-1.53791600$

$-2.88262600$

$-1.10584100$

4. 95093600

4. 95495100

2.16644700

0.35195500

2. 52267900

0.19908500

$-1.54541300$

$-1.43094300$

$-2.58035500$

$-3.09198600$

$-3.74612000$

$-2.67502000$

$-3.92762200$

$-4.82325900$

$-3.21687900$

$-4.06341600$

$-0.91710800$

$-2.13481100$

$-0.68190000$

$-2.31212600$

$-2.50621400$

$-2.86536200$

$-0.69240100$

$-0.33515900$

$-1.93674500$

$-0.97678800$

$-4.19120300$

$-4.77524900$
$Y$

0.26589600

$-1.06308900$

$-1.51853500$

$-0.59589300$

0.78533900

1. 22846700

2. 53645500

3. 40197800

3.09684400

1.76875400

1. 39210300

2. 31368900

1.67544300

2.83066400

0.52525500

$-0.89136900$

$-1.22924800$

$-1.14162900$

$-2.54233700$

$-2.03941800$

$-2.53951200$

0.61432400

$-2.24341300$

$-2.57399400$

$-0.94243000$

4. 44664500

3. 90371300

3.17487900

3. 33912000

2. 01453100

3.53965200

2.48639800

1.34654100

0.69044100

0.54535600

$-1.01637200$

$-1.60092600$

$-1.36662600$

$-0.10652800$

$-2.83584400$

$-3.31073500$

$-2.08988400$

$-3.05926700$

$-3.51550700$

$-1.78502600$

$-2.34304000$

0.39932900

$-1.49594700$

$-2.10759500$
Z

$-0.25757400$

$-0.08702200$

0.18803900

0.27345500

0.09746500

$-0.17053300$

$-0.35094300$

$-0.26091400$

$-0.00480500$

0.18532600

0.45300200

0.51596700

1.27224500

$-0.86686000$

0.60211200

0.87041600

0.29072800

$-1.17313800$

0.76627600

$-1.97111100$

2. 22755300

$-0.46321100$

$-0.22754100$

0.32756900

0.49038500

$-0.40704100$

0.04530400

1. 11864000

$-1.35197100$

$-1.52086800$

$-0.77672200$

1.45823600

2.25313500

$-0.47227300$

1. 00475400

1.95284300

0.51525000

$-1.46777100$

$-1.47044600$

0.14804800

0.61269500

$-3.00092700$

$-1.56510500$

2.48797400

2.40374700

2.90706300

0.53667500

$-1.93704500$

$-2.39231800$ 
Atom

$\mathrm{C}$

C

C

C

C

C

$\mathrm{N}$

C

C

C

$\mathrm{N}$

C

C

C

C

C

$\mathrm{N}$

C

C

C

C

$\mathrm{H}$

$\mathrm{Cl}$

$\mathrm{H}$

$\mathrm{H}$

$\mathrm{H}$

$\mathrm{H}$

$\mathrm{H}$

$\mathrm{H}$

$\mathrm{H}$

$\mathrm{H}$

$\mathrm{H}$

$\mathrm{H}$

$\mathrm{H}$

$\mathrm{H}$

$\mathrm{H}$

$\mathrm{H}$

$\mathrm{H}$

$\mathrm{H}$

$\mathrm{H}$

$\mathrm{H}$

$\mathrm{H}$

$\mathrm{H}$

$\mathrm{H}$

$\mathrm{H}$

$\mathrm{H}$

$\mathrm{H}$

O

$\mathrm{H}$ $\mathrm{x}$

$-4.43392600$

$-4.51251000$

$-3.62190000$

$-2.62656100$

$-2.47499600$

$-3.42419400$

$-3.45023700$

$-2.53984800$

$-1.53717900$

$-1.45422300$

$-0.47892200$

0.39353100

1. 77681700

0.49189500

1. 77426900

3. 17022200

4. 07246700

5.09920800

4.62138000

5.36235900

5.40931700

$-5.13489800$

$-5.76954000$

$-3.74044100$

$-1.96970300$

$-2.57037400$

$-0.80286000$

$-0.05758600$

1. 13741000

$-0.49162100$

0.92548400

2. 20007200

2. 44024700

1.13376600

1. 34844300

3. 08046300

3.59654300

6.05635400

4. 78892100

3.77784500

5.26388300

6.29053600

5.51805200

5.65831000

4.82036300

6.34617400

0.06421100

4. 26648900

4. 42554500
$Y$

0.24638800

$-1.08106300$

$-1.68286600$

$-0.92258300$

0.44939400

1. 05224500

2. 38264800

3. 12807800

2. 64707100

1. 29196100

0.80907100

$-0.33703500$

0.15040000

$-1.27561000$

0.94776300

1. 40220200

0.28701100

0.05305900

0.44088300

$-1.40726600$

$-0.76046800$

0.71586100

$-2.06941000$

$-2.72646300$

$-1.37682600$

4. 19244900

3.33265300

$-0.86349000$

$-2.12633500$

$-1.64820800$

$-0.74723000$

0.76341700

$-0.71123900$

1.83194900

0.33502000

2. 05649600

2.02419400

0.51541900

0.53303600

0.60744500

1. 34019300

$-1.45447600$

$-1.99396100$

$-0.61952500$

$-1.67671200$

$-0.89089600$

1. 56401400

$-1.91875800$

$-2.85317600$
Z

0.97594900

0.66122400

$-0.24982600$

$-0.80603700$

$-0.48274800$

0.38922800

0.68380800

0.09020800

$-0.76104200$

$-1.04157100$

$-1.88636200$

$-1.56155900$

$-1.13098600$

$-2.75829100$

0.16632200

0.58455300

0.90595700

$-0.10864000$

2. 25374000

$-0.44695200$

2.75606600

1. 65389400

1.37297600

$-0.50901700$

$-1.53574100$

0.30933000

$-1.16855100$

$-0.71581600$

$-2.52128300$

$-3.05489100$

$-3.61436900$

$-1.93964800$

$-1.02936900$

0.06740600

0.96779900

1.45734600

$-0.21444800$

0.18566200

$-1.04174600$

2. 93082100

2.31656500

$-1.03296700$

0.46527000

3.81083600

2.65636500

2.20876800

$-2.28707700$

$-1.18327100$

$-1.34121300$ 
Atom

C

C

C

$\mathrm{C}$

C

C

$\mathrm{N}$

C

$\mathrm{C}$

C

$\mathrm{N}$

C

$\mathrm{C}$

C

C

C

$\mathrm{N}$

C

C

C

C

$\mathrm{H}$

$\mathrm{Cl}$

$\mathrm{H}$

$\mathrm{H}$

$\mathrm{H}$

$\mathrm{H}$

$\mathrm{H}$

$\mathrm{H}$

$\mathrm{H}$

$\mathrm{H}$

$\mathrm{H}$

$\mathrm{H}$

$\mathrm{H}$

$\mathrm{H}$

$\mathrm{H}$

$\mathrm{H}$

$\mathrm{H}$

$\mathrm{H}$

$\mathrm{H}$

$\mathrm{H}$

$\mathrm{H}$

$\mathrm{H}$

$\mathrm{H}$

$\mathrm{H}$

$\mathrm{H}$

$\mathrm{H}$

O

$\mathrm{H}$

\section{$\mathrm{x}$}

$-3.17203000$

$-3.77315500$

$-3.16296100$

$-1.93984900$

$-1.28255600$

$-1.90642200$

$-1.36250300$

$-0.18539100$

0.53382400

0.00567900

0.64884100

2.09545000

2.86521900

2.43196900

2.55164000

2.88794600

2.07836700

2.84606500

1.08338900

2.03775600

$-0.02524000$

$-3.63713600$

$-5.34715700$

$-3.65771200$

$-1.48700500$

0.25090300

1.49737700

2. 35158300

3.49728400

1.86785800

2.19787600

3.93855000

2. 60595800

3.09189700

1.48165500

2.73817000

3.95279700

3.23384200

3.71457100

1.55657800

0.64002700

1.20139400

1.61585100

$-0.76960900$

0.36915300

$-0.51835300$

0.23747000

2.81864000

3.48064200
Y

$-0.65688000$

$-0.01704300$

1.07283600

1.50793200

0.88886400

$-0.21803000$

$-0.90187600$

$-0.47831300$

0.59282200

1.30903800

2.36781600

2.57000800

1.40316600

3.91389400

1.19508800

$-0.22239300$

$-1.22750400$

$-2.18682500$

$-1.84988000$

$-2.73778000$

$-0.88158400$

$-1.49699700$

$-0.54784600$

1.55500500

2.34503800

$-1.02163200$

0.84376000

2.61986700

4.12031100

4.71769400

3.91061000

1.56868600

0.48566100

1.92196000

1.35630000

$-0.30076800$

$-0.41697700$

$-3.02593800$

$-1.66539400$

$-2.26777000$

$-2.69199900$

$-3.34543500$

$-1.89312900$

$-1.38194100$

$-0.03179400$

$-0.48931100$

2.75454200

$-3.58950300$

$-3.03950800$
Z

$-0.80500200$

0.24349300

0.89443400

0.45172000

$-0.63812200$

$-1.27284900$

$-2.31889800$

$-2.73541300$

$-2.19344900$

$-1.12498100$

$-0.56513000$

$-0.66376500$

$-0.02624800$

$-0.03299300$

1. 45557100

1.91947100

1.22785500

0.45167600

2.09289000

$-0.72064400$

2. 48588400

$-1.30378600$

0.79938200

1.72683100

0.96840300

$-3.57018100$

$-2.61312000$

$-1.72601700$

$-0.14225100$

$-0.50902800$

1. 03576500

$-0.17173400$

$-0.56451500$

2.07165500

1. 62994200

3.00451200

1. 74267400

1.05650800

0.03250900

2. 99832400

1.55644300

$-0.36475000$

$-1.28297100$

3.10982900

3.04899400

1.59361700

0.27009700

$-1.53948500$

$-1.96819400$ 
Atom

$\mathrm{C}$

$\mathrm{C}$

C

C

C

C

$\mathrm{N}$

C

C

C

$\mathrm{N}$

C

C

C

C

C

$\mathrm{N}$

C

C

C

C

$\mathrm{H}$

$\mathrm{Cl}$

$\mathrm{H}$

$\mathrm{H}$

$\mathrm{H}$

$\mathrm{H}$

$\mathrm{H}$

$\mathrm{H}$

$\mathrm{H}$

$\mathrm{H}$

$\mathrm{H}$

$\mathrm{H}$

$\mathrm{H}$

$\mathrm{H}$

$\mathrm{H}$

$\mathrm{H}$

$\mathrm{H}$

$\mathrm{H}$

$\mathrm{H}$

$\mathrm{H}$

$\mathrm{H}$

$\mathrm{H}$

$\mathrm{H}$

$\mathrm{H}$

$\mathrm{H}$

$\mathrm{H}$

O

$\mathrm{H}$ $\mathrm{x}$

$-3.96813800$

$-3.01760500$

$-1.66656400$

$-1.28861700$

$-2.22747600$

$-3.59366800$

$-4.58819500$

$-4.22843000$

$-2.90381600$

$-1.87551500$

$-0.54327100$

0.37015100

1.81121100

0.07280000

2.25016800

2.46117800

3.71778800

4.90654500

3.92022200

4.84531700

2.77245500

$-5.01035500$

$-3.47559100$

$-0.94861700$

$-0.26197800$

$-5.02513000$

$-2.69574200$

0.20054900

0.68272300

$-0.98078600$

0.30197100

1.89940800

2.46806800

3.17149600

1.48648800

1.64731100

2.38426300

5.14307300

5.75191400

4.82513900

4.14105700

5.81529700

4.08576700

3.07619800

2.49988000

1.88087900

$-0.49000300$

4.56796300

4.54689000
Y

1.19821400

2.18048300

1.91899300

0.63509200

$-0.42522900$

$-0.13229300$

$-1.06277700$

$-2.28469600$

$-2.69850900$

$-1.78430200$

$-2.13884400$

$-2.08624600$

$-2.15528100$

$-3.20523600$

$-0.92731200$

0.36386700

0.52386000

0.48836900

$-0.32715100$

1.36897000

$-0.29587200$

1.40355800

3.82002800

2.72848000

0.43201900

$-3.02162400$

$-3.73969400$

$-1.12915700$

$-3.08823600$

$-3.19772700$

$-4.17757100$

$-3.05375900$

$-2.31893000$

$-1.16065200$

$-0.73466600$

0.49356900

1.21832800

$-0.53601500$

0.81213900

0.03992500

$-1.37321700$

1.29327000

0.99643000

$-0.78840000$

0.73641200

$-0.81093900$

$-3.06467800$

2.70243800

3.23431400
Z

0.17320500

0.19637000

$-0.11314300$

$-0.40668200$

$-0.39658600$

$-0.14562600$

$-0.21603500$

$-0.54503000$

$-0.77604900$

$-0.67820300$

$-0.88228100$

0.27959400

$-0.22360400$

1.27392000

$-1.02379600$

$-0.20969900$

0.51606300

$-0.32174300$

1.68321500

$-1.55517800$

2. 68179900

0.38201900

0.60333100

$-0.12763700$

$-0.68478700$

$-0.61040300$

$-0.99359700$

0.78248700

2.17277900

1. 56194400

0.82763000

$-0.84590100$

0.63432000

$-1.56783800$

$-1.78629300$

0.51063300

$-0.88790300$

$-0.66523600$

0.29621500

2.17960100

1. 40320100

$-2.06361700$

$-2.25573600$

3.60674000

2. 91212300

2. 31373800

$-1.29578700$

$-1.17073300$

$-1.97027200$ 
Atom

$\mathrm{C}$

C

C

C

C

C

$\mathrm{N}$

C

C

C

$\mathrm{N}$

C

C

C

C

C

$\mathrm{N}$

C

C

C

C

$\mathrm{H}$

$\mathrm{Cl}$

$\mathrm{H}$

$\mathrm{H}$

$\mathrm{H}$

$\mathrm{H}$

$\mathrm{H}$

$\mathrm{H}$

$\mathrm{H}$

$\mathrm{H}$

$\mathrm{H}$

$\mathrm{H}$

$\mathrm{H}$

$\mathrm{H}$

$\mathrm{H}$

$\mathrm{H}$

$\mathrm{H}$

$\mathrm{H}$

$\mathrm{H}$

$\mathrm{H}$

$\mathrm{H}$

$\mathrm{H}$

$\mathrm{H}$

$\mathrm{H}$

$\mathrm{H}$

$\mathrm{H}$

O

$\mathrm{H}$

\section{$\mathrm{x}$}

4.73055300

4.33381600

2.98912300

2.06709500

2.42287600

3.78530900

4.26396000

3.37621400

2. 02448100

1.50311500

0.16487000

$-0.54837700$

$-1.98914600$

0.10660000

$-2.80106700$

$-3.16655200$

$-4.33875300$

$-5.58074400$

$-4.22134500$

$-5.84183500$

$-2.99870100$

5.75924000

5.51050400

2.69369600

1.03975100

3.74867500

1.37911300

$-0.58899000$

$-0.42061200$

1.15365900

0.05488300

$-1.95586300$

$-2.46941000$

$-3.71688200$

$-2.22924200$

$-2.31635900$

$-3.33989700$

$-5.63835900$

$-6.40073500$

$-5.11915900$

$-4.24808300$

$-6.81983900$

$-5.09587400$

$-3.09337900$

$-2.91349400$

$-2.06626900$

$-0.27344100$

$-5.82059400$

$-6.01597100$
$\mathrm{Y}$

$-0.11732500$

$-1.42054300$

$-1.76510300$

$-0.76546500$

0.60743000

0.93088300

2.19552900

3.16491300

2.98203300

1.70427100

1.59311000

0.57233000

1.05319400

0.28795500

1.12621300

$-0.22845800$

$-0.93135300$

$-0.17733500$

$-1.50514000$

0.45904800

$-2.39136200$

0.15402100

$-2.70072500$

$-2.80437300$

$-1.05007100$

4.17773600

3.84693000

$-0.35273300$

$-0.52805200$

0.00316100

1.17627500

2.03932100

0.38687600

1.69667000

1.70202900

$-0.91473300$

$-0.07543600$

0.62911900

$-0.86727300$

$-2.11387900$

$-0.72641700$

0.95594100

1.23913800

$-2.95140900$

$-3.10467900$

$-1.82109700$

2.50037700

$-0.53580900$

$-0.10488400$
Z

$-0.50244900$

$-0.41639600$

$-0.17815300$

$-0.01356600$

$-0.07099800$

$-0.34737100$

$-0.49684200$

$-0.37871200$

$-0.09173000$

0.09859900

0.36722500

1.14086900

1.33018100

2. 48894100

0.03388000

$-0.60195600$

$-0.08590900$

$-0.17987500$

1. 25035100

$-1.53127100$

1.44205400

$-0.69953400$

$-0.61580100$

$-0.13231100$

0.15151600

$-0.50639000$

0.00949300

0.55724900

2.98956700

2. 37743100

3. 12215700

1. 81006400

2.05011200

0.21651700

$-0.70129600$

$-0.54211800$

$-1.67134000$

0.57601900

0.04444700

1. 40872300

2.03530900

$-1.47955900$

$-1.73418200$

2.37591800

0.61686000

1.49583400

0.44652800

$-2.53759400$

$-3.37334000$ 
Atom

C

C

C

C

C

C

$\mathrm{N}$

C

$\mathrm{C}$

C

$\mathrm{N}$

C

C

C

C

C

N

C

C

C

C

$\mathrm{H}$

$\mathrm{Cl}$

$\mathrm{H}$

H

$\mathrm{H}$

$\mathrm{H}$

$\mathrm{H}$

$\mathrm{H}$

$\mathrm{H}$

$\mathrm{H}$

$\mathrm{H}$

$\mathrm{H}$

$\mathrm{H}$

$\mathrm{H}$

$\mathrm{H}$

$\mathrm{H}$

$\mathrm{H}$

$\mathrm{H}$

$\mathrm{H}$

$\mathrm{H}$

$\mathrm{H}$

$\mathrm{H}$

$\mathrm{H}$

$\mathrm{H}$

$\mathrm{H}$

$\mathrm{H}$

$\mathrm{O}$

$\mathrm{H}$

\section{$\mathrm{x}$}

$-3.59743100$

$-4.41200200$

$-4.05052400$

$-2.84800000$

$-1.96748200$

$-2.35547500$

$-1.60663200$

$-0.47850400$

0.01865200

$-0.69053600$

$-0.27379800$

0.98636800

2.10092400

1.42440100

2.52633600

3.57141300

4.00862600

3.13763200

4.58030500

1.96838100

5.36230700

$-3.87394700$

$-5.94960800$

$-4.71633400$

$-2.60399800$

0.11557700

0.98070000

0.73854800

2.13102700

0.57043400

1.93134700

2.96050200

1.75670800

2.93304500

1.66447700

4.46107800

3.17414300

3.76220000

2.73169600

3.81885700

5.25064000

1.72351600

2.27684000

5.86980000

4.70249400

6.11408200

$-0.85392100$

0.83558000

0.28123500
$\mathrm{Y}$

$-1.07637000$

$-0.57942500$

0.54990700

1.15878500

0.68634400

$-0.45023000$

$-0.98976500$

$-0.35828600$

0.76200500

1.30331500

2. 34330400

3.09545100

2. 36924400

3.59989800

1.02592300

0.32676100

$-0.99038700$

$-2.11763600$

$-0.99087500$

$-2.44973000$

$-2.26429300$

$-1.94121400$

$-1.34739500$

0.92870400

2.03342300

$-0.77369400$

1.15222500

3.98830000

4.41802100

3.97527600

2.84352000

3.04788800

2.21413100

1.16315800

0.36202600

0.96719000

0.22591900

$-3.01850500$

$-1.96439400$

$-0.84292800$

$-0.12529000$

$-3.50927000$

$-2.32440400$

$-2.17314900$

$-3.13760200$

$-2.44796200$

2.56310000

$-1.65255400$

$-1.58512200$
Z

0.67442800

$-0.30457000$

$-1.06265500$

$-0.81072500$

0.19275700

0.95169600

1. 95648300

2. 22988400

1. 55714800

0.48567400

$-0.27269300$

$-0.27229800$

$-1.04165400$

1.10239800

$-0.46052500$

$-1.33333500$

$-0.89470400$

$-1.20255900$

0.44112600

$-0.26518100$

0.74802800

1. 26272400

$-0.63024400$

$-1.82642800$

$-1.40137800$

3.03954600

1.84740600

$-0.85252600$

0.95666900

1. 66740600

1.70026300

$-1.09719800$

$-2.07068700$

0.54853000

$-0.38247500$

$-1.40245000$

$-2.34967900$

$-1.25202900$

$-2.20990800$

1. 22811300

0.50233600

$-0.40197100$

0.77927800

1. 71113500

0.80318400

$-0.02421400$

$-1.06260300$

$-0.56531600$

0.22219900 
Atom

C

C

C

$\mathrm{C}$

C

C

$\mathrm{N}$

C

$\mathrm{C}$

C

$\mathrm{N}$

C

C

$\mathrm{C}$

C

C

$\mathrm{N}$

C

C

C

C

$\mathrm{H}$

$\mathrm{Cl}$

$\mathrm{H}$

$\mathrm{H}$

$\mathrm{H}$

$\mathrm{H}$

$\mathrm{H}$

$\mathrm{H}$

$\mathrm{H}$

$\mathrm{H}$

$\mathrm{H}$

$\mathrm{H}$

$\mathrm{H}$

$\mathrm{H}$

$\mathrm{H}$

$\mathrm{H}$

$\mathrm{H}$

$\mathrm{H}$

$\mathrm{H}$

$\mathrm{H}$

$\mathrm{H}$

$\mathrm{H}$

$\mathrm{H}$

$\mathrm{H}$

$\mathrm{H}$

$\mathrm{H}$

$\mathrm{O}$

$\mathrm{H}$

\section{$\mathrm{x}$}

3.31487200

2. 52894400

1. 39686700

1.10464700

1. 91163100

3. 00624400

3. 77041000

3. 41864400

2.37658400

1. 62881600

0.67256500

$-0.31075900$

$-0.96389900$

0.23139600

$-1.46554100$

$-2.45322800$

$-2.94089400$

$-4.00546600$

$-3.38153800$

$-4.16802900$

$-2.22698400$

4.16755400

2.90897200

0.76423000

0.20460900

4. 01451700

2. 21258500

$-1.08060200$

$-0.59517000$

0.72123200

0.94490400

$-0.24648400$

$-1.79246200$

$-0.62584100$

$-1.92929500$

$-1.95218300$

$-3.28625700$

$-3.76844800$

$-4.97621700$

$-3.95739000$

$-4.05990800$

$-4.79873600$

$-3.18118000$

$-2.60625500$

$-1.53851400$

$-1.66395000$

0.31774700

$-4.76511700$

$-4.79386500$
$Y$

$-1.56653600$

$-2.43558200$

$-2.00030200$

$-0.66149900$

0.28369500

$-0.18226100$

0.62581400

1.89519900

2.47865000

1.69629500

2. 19891500

3. 23358200

2. 97153500

4.65579200

1.53950000

1.07665700

$-0.28094400$

$-0.28101700$

$-0.92116700$

$-1.58950900$

$-1.35784500$

$-1.90845600$

$-4.14375900$

$-2.71443500$

$-0.34383700$

2. 53707400

3.54377600

3.13291800

5.36539100

4.80216200

4.89101800

3.21225600

3. 67987200

0.83765800

1. 47094500

1.08137700

1.79953000

0.47791100

0.00294900

$-1.80150000$

$-0.25446200$

$-1.40085000$

$-1.91524300$

$-1.92292100$

$-1.99729600$

$-0.51116300$

1. 53260600

$-2.57183100$

$-3.39634700$
Z

$-0.51987800$

0.18598000

0.90316500

0.92164500

0.24453300

$-0.52981100$

$-1.31809700$

$-1.35588500$

$-0.61975700$

0.24697600

1. 08804300

0.73630500

$-0.62880600$

0.84822300

$-0.82668700$

0.24027700

0.00186500

$-0.99984300$

1. 24430600

$-1.75431200$

2.13746500

$-1.09180100$

0.17856700

1. 41529100

1.43505700

$-1.99990300$

$-0.69722200$

1. 50691000

0.76522300

1. 81191400

0.05616600

$-1.42032200$

$-0.73470500$

$-0.83358700$

$-1.81550200$

1.21229300

0.32390200

$-1.74936900$

$-0.55473700$

0.96243100

1.80689100

$-2.63317000$

$-2.10815500$

2. 99225800

1.57723100

2. 54017700

1.75362700

$-0.92311000$

$-1.41770200$ 


\section{HCQ-MD1- Structure}

$\omega B 97 x-D / 6-31 G(d, p)$ - Water fully optimized XYZ coordinates

\section{Atom}

$\mathrm{C}$

$\mathrm{C}$

$\mathrm{C}$

$\mathrm{C}$

$\mathrm{C}$

C

$\mathrm{N}$

C

C

C

$\mathrm{N}$

C

C

C

C

C

$\mathrm{N}$

C

C

C

C

$\mathrm{H}$

$\mathrm{Cl}$

$\mathrm{H}$

$\mathrm{H}$

$\mathrm{H}$

$\mathrm{H}$

$\mathrm{H}$

$\mathrm{H}$

$\mathrm{H}$

$\mathrm{H}$

$\mathrm{H}$

$\mathrm{H}$

$\mathrm{H}$

$\mathrm{H}$

$\mathrm{H}$

$\mathrm{H}$

$\mathrm{H}$

$\mathrm{H}$

$\mathrm{H}$

$\mathrm{H}$

$\mathrm{H}$

$\mathrm{H}$

$\mathrm{H}$

$\mathrm{H}$

$\mathrm{H}$

$\mathrm{H}$

O

$\mathrm{H}$

\section{$\mathrm{x}$}

3. 95320300

3. 50473000

2. 24818700

1.44539000

1.86354600

3.14213800

3. 65551100

2.88520300

1. 60712600

1.04214400

$-0.20636400$

$-1.17577300$

$-2.57416000$

$-1.04623300$

$-2.75520600$

$-3.72679300$

$-3.26130300$

$-2.07214700$

$-4.32491800$

$-2.04165200$

$-5.02622700$

4.91958700

4.50899700

1.92305000

0.48061800

3.29214400

1.07485000

$-0.97963100$

$-1.76004300$

$-0.04075200$

$-1.24503700$

$-2.73231900$

$-3.30853600$

$-1.78041800$

$-3.08292800$

$-3.88548400$

$-4.70475900$

$-1.92831900$

$-1.18556600$

$-5.07506500$

$-3.90927100$

$-1.30211100$

$-3.01726700$

$-5.91912100$

$-5.33521300$

$-4.37979200$

$-0.59194000$

$-1.67618900$

$-1.77636300$

\section{Y}

$-0.54238800$

$-1.66904600$

$-1.71433700$

$-0.60117300$

0.58733200

0.62045200

1. 71246800

2.78242100

2.88130500

1.77401500

1.78219200

2.85409300

2. 23473000

3. 92850900

1.23240600

0.08224900

$-1.17904000$

$-1.73903100$

$-2.14388200$

$-1.84740700$

$-2.76089900$

$-0.50577900$

$-3.10604100$

$-2.61735900$

$-0.65235600$

3.65651100

3. 81960000

3.30128500

4.73572900

4.35297500

3. 49284900

1.73024000

3. 04042800

0.80228800

1. 74866900

0.01179100

0.29255600

$-2.73976400$

$-1.15893800$

$-1.63472100$

$-2.94943300$

$-2.60427900$

$-2.15038400$

$-3.30195500$

$-1.99030300$

$-3.46935700$

0.91996900

$-0.58360700$

$-0.62414400$
$\mathbf{Z}$

0.48508700

$-0.14779100$

$-0.78175600$

$-0.74391800$

$-0.09772600$

0.52151600

1.16371300

1.17369500

0.60903500

$-0.02146300$

$-0.53903800$

$-0.33132200$

$-0.30767100$

$-1.40391300$

0.83372900

0.52475200

1.09292600

0.46116900

1. 34102500

$-1.06439000$

0.12294800

0.97268400

$-0.17299900$

$-1.28365900$

$-1.23854200$

1.67567800

0.67705700

0.65113900

$-1.23015600$

$-1.41125900$

$-2.38586000$

$-1.26664900$

$-0.25250100$

1.07584000

1.74015000

$-0.56407300$

0.96732100

0.87553600

0.74553600

1.95572100

1.95665500

$-1.35080400$

$-1.45392100$

0.44264900

$-0.58931700$

$-0.40145300$

$-0.90370500$

$-1.61893400$

$-2.57285400$ 


\section{HCQ-MD2 - Structure}

$\omega \mathrm{B} 97 \mathrm{x}-\mathrm{D} / 6-31 \mathrm{G}(\mathrm{d}, \mathrm{p})$ - Water fully optimized XYZ coordinates

\section{Atom}

$\mathrm{C}$

$\mathrm{C}$

$\mathrm{C}$

$\mathrm{C}$

$\mathrm{C}$

C

$\mathrm{N}$

C

$\mathrm{C}$

$\mathrm{C}$

$\mathrm{N}$

C

C

C

C

C

$\mathrm{N}$

C

C

C

C

$\mathrm{H}$

$\mathrm{Cl}$

$\mathrm{H}$

$\mathrm{H}$

$\mathrm{H}$

$\mathrm{H}$

$\mathrm{H}$

$\mathrm{H}$

$\mathrm{H}$

$\mathrm{H}$

$\mathrm{H}$

$\mathrm{H}$

$\mathrm{H}$

$\mathrm{H}$

$\mathrm{H}$

$\mathrm{H}$

$\mathrm{H}$

$\mathrm{H}$

$\mathrm{H}$

$\mathrm{H}$

$\mathrm{H}$

$\mathrm{H}$

$\mathrm{H}$

$\mathrm{H}$

$\mathrm{H}$

$\mathrm{H}$

$\mathrm{O}$

$\mathrm{H}$

\section{$\mathrm{x}$}

3.62178800

4. 21724100

3. 70156600

2. 57428000

1.92138000

2.45226400

1.90454000

0.82753700

0.21594700

0.73403400

0.19901400

$-1.17395300$

$-2.04026100$

$-1.18074500$

$-1.99362300$

$-2.57966900$

$-2.29973100$

$-3.00772900$

$-2.26353300$

$-4.51399600$

$-3.57908400$

4.01745300

5.65619200

4. 19116200

2.19734700

0.38480700

$-0.65335800$

$-1.57546700$

$-2.20443600$

$-0.59957600$

$-0.75278200$

$-1.71170600$

$-3.07237900$

$-2.54486900$

$-0.95741000$

$-3.65659700$

$-2.11751300$

$-2.83420200$

$-2.56539600$

$-1.51972200$

$-1.87344800$

$-4.95369200$

$-4.98296100$

$-3.39917600$

$-4.04080500$

$-4.29549300$

0.62201300

$-4.72643600$

$-5.67299100$

\section{$Y$}

$-1.09134600$

$-0.64724700$

0.44215700

1. 07157200

0.65399000

$-0.45306600$

$-0.95617600$

$-0.33490000$

0.77005100

1. 29312200

2. 36518300

2.84584200

2.34889100

4. 36851700

0.84207200

0.02091600

$-1.39893100$

$-2.06554100$

$-2.13056900$

$-1.83116900$

$-2.25569000$

$-1.92874700$

$-1.44312500$

0.76827200

1. 90846000

$-0.72601000$

1. 20052400

2.45379100

4.73684700

4. 71803000

4.79790100

2.87143800

2.66754900

0.61417600

0.52086900

0.24150700

0.32636300

$-3.13948600$

$-1.77153300$

$-1.63424700$

$-3.13226300$

$-2.67587200$

$-1.78668400$

$-2.69917800$

$-1.27520900$

$-2.89047500$

2.61616100

$-0.61805500$

$-0.46077900$

\section{$\mathbf{Z}$}

0.51757100

$-0.63181400$

$-1.36177000$

$-0.90062100$

0.28518100

1.00339700

2.14674700

2. 58380600

1. 98082800

0.80072000

0.15935500

0.29173900

$-0.87900500$

0.36862900

$-1.12263200$

0.02273100

$-0.13100000$

$-1.21556900$

1. 12731100

$-1.34850200$

1.90233400

1.07859100

$-1.23017900$

$-2.27130600$

$-1.47822700$

3. 49659200

2. 45708700

1. 22717100

0.46634700

1. 22334400

$-0.54285700$

$-1.78543000$

$-0.69355500$

$-2.03859700$

$-1.27697500$

0.11288900

0.96506200

$-1.10043100$

$-2.17547400$

1.76249400

0.91793500

$-1.89453700$

$-0.35856200$

2. 88372700

2.05152600

1.37600900

$-0.72071000$

$-2.05670000$

$-2.08718800$ 